\title{
Stereoselectivity of Conformationally Restricted Glucosazide Donors
}

\author{
Stefan van der Vorm, Herman S. Overkleeft, Gijsbert A. van der Marel, and Jeroen D. C. Codée*(i) \\ Leiden Institute of Chemistry, Leiden University, Einsteinweg 55, 2333 CC Leiden, The Netherlands
}

\section{Supporting Information}

ABSTRACT: Glycosylations of 4,6-tethered glucosazide donors with a panel of model acceptors revealed the effect of acceptor nucleophilicity on the stereoselectivity of these donors. The differences in reactivity among the donors were evaluated in competitive glycosylation reactions, and their relative reactivities were found to be reflected in the stereoselectivity in glycosylations with a set of fluorinated alcohols as well as carbohydrate acceptors. We found that the 2 -azido-2-deoxy moiety is more $\beta$-directing than its $\mathrm{C}$-2-Obenzyl counterpart, as a consequence of increased destabilization of anomeric charge development by the electronwithdrawing azide. Additional disarming groups further decreased the $\alpha$-selectivity of the studied donors, whereas

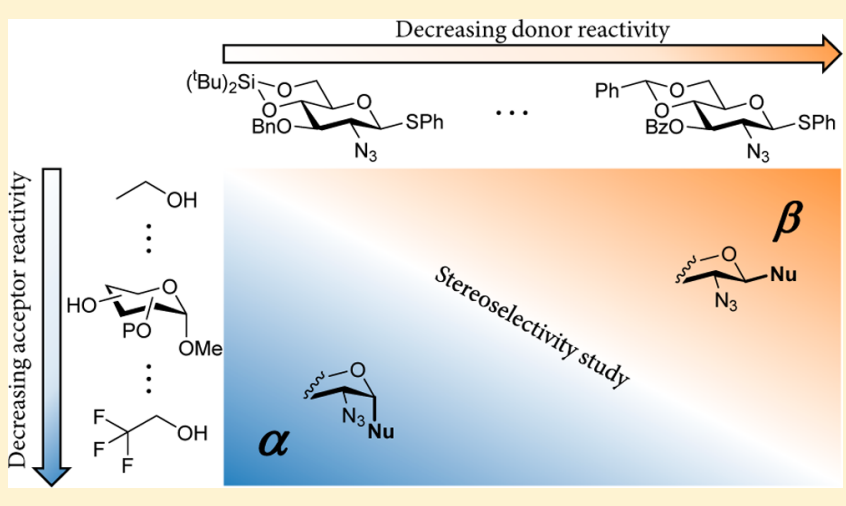
substitution of the 4,6-benzylidene acetal with a 4,6-di-tertbutyl silylidene led to a slight increase in $\alpha$-selectivity. The C-2-dinitropyridone group was also explored as an alternative for the nonparticipating azide group, but this protecting group significantly increased $\beta$-selectivity. All studied donors exhibited the same acceptor-dependent selectivity trend, and good $\alpha$-selectivity could be obtained with the weakest acceptors and most reactive donors.

\section{INTRODUCTION}

Glucosamine is a key constituent in numerous important oligosaccharides and glycoconjugates, where it can be either $\alpha$ or $\beta$-linked. ${ }^{1-5}$ Whereas the former type of linkage can be reliably installed through the use of neighboring-group participation of a C-2-amide- or carbamate-based protecting group, the latter type continues to present a synthetic challenge. $^{6-8}$ A thorough understanding of the glycosylation mechanism and the influence of both reaction partners and reaction conditions on glycosylation stereoselectivity is needed to enable reliable and predictable glycosylation reactions. The in-depth research conducted on conformationally restricted benzylidene mannose and glucose donors has provided important insight into the glycosylation mechanisms of this type of 1,2-cis-selective donor. ${ }^{9-17}$ To construct 1,2-cis linkages of glucosamine donors, the C-2-amino group is most commonly masked as the nonparticpating azide. ${ }^{18,19}$ Notably, benzylidene glucosazides have not been systematically investigated with respect to the stereoselectivity of glycosylations in which they are employed. The extrapolation of the stereoselectivity of benzylidene glucose donors to their glucosazide counterparts suggests that benzylidene or analogously protected glucosazides might represent an attractive class of 1,2-cis-selective glucosamine donor synthons. ${ }^{20,21}$

Recently, we advocated the use of a comprehensive set of partially fluorinated ethanols, of gradually decreasing nucleophilicity, that can be used to map how the stereoselectivity of a given glycosylation system is dependent on the nucleophilicity of the acceptor. ${ }^{22}$ The stereoselectivity of the benzylidene glucose donor system has proved to be greatly affected by the reactivity of the nucleophile. ${ }^{23-27}$ In light of the demand for 1,2-cis-selective glucosaminylations but also with the aim in mind of furthering the understanding of the stereoelectronic effects exerted by the azido group, we set out to systematically evaluate a series of glucosazide donors in a set of glycosylation reactions involving the toolset of partially fluorinated ethanols and a selection of carbohydrate acceptors. As we describe herein, changes in the structure and reactivity of the donor can be effectively mapped using our panel of model acceptors, and we observed a clear reactivity-selectivity relationship for the stereoselectivity of the glycosylations of all donors studied. Differences among the donors and the stereochemical variation in the glycosylation outcome can be explained on the basis of competition experiments and the characterization of the reactive intermediates involved.

\section{RESULTS AND DISCUSSION}

The set of (partially) fluorinated ethanol acceptors that we recently employed to relate the glycosylation stereoselectivity to the acceptor nucleophilicity is depicted in Figure 1 (compounds 6-11). Glycosylating these acceptors with benzylidene mannose, benzylidene glucose, and mannuronic acid donors, as well as fucosazide donors bearing various protecting groups, established the dependence of the stereoselectivity of the glycosylations with these donors on the

Received: February 27, 2017

Published: April 12, 2017 


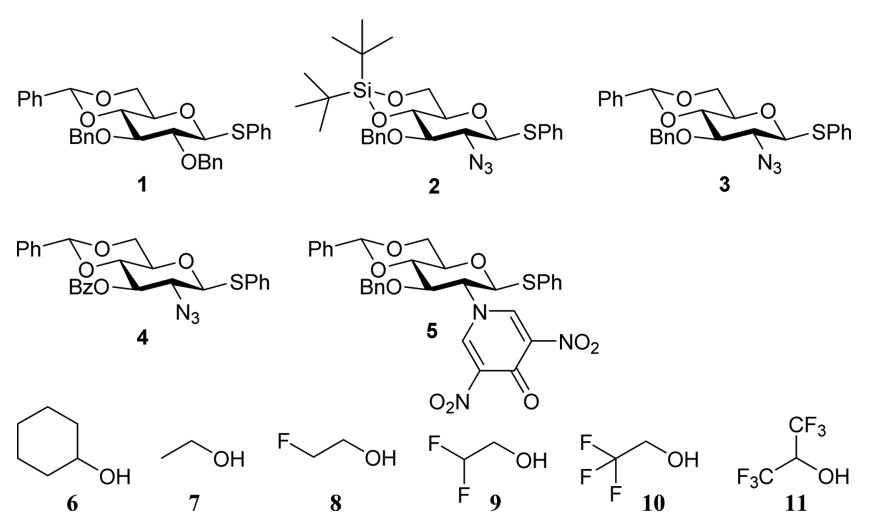

Figure 1. Glucose-configured donors 1-5 and model acceptors 6-11 used in this study.

nucleophilicity of the acceptor. ${ }^{22,28}$ For benzylidene-protected glucose donor 1, the gradual decrease in acceptor nucleophilicity going from ethanol to monofluoroethanol (MFE), difluoroethanol (DFE), trifluoroethanol (TFE), and hexafluoroisopropanol (HFIP) led to a gradual shift of the stereoselecivity from high $\beta$-selectivity to exclusive $\alpha$-selectivity (see Table 3 below). Here, we present our results from investigating the set of conformationally restricted glucosamine donors depicted in Figure $1(\mathbf{1 - 5})$. Variation in the structure of these donors is found in the cyclic protecting groups (benzylidene vs silylidene), in the functionality at the $\mathrm{C}-3-\mathrm{OH}$ (benzyl vs benzoyl), and in the nature of the $\mathrm{C}-2-\mathrm{N}$-protecting group [azide vs the dinitropyridone (DNPY) group]. The DNPY group is introduced here as a nonparticipating $N$-protecting group. $^{29,30}$ The reactivity and selectivity of the set of glucosamine donors are related to the corresponding properties of the well-studied benzylidene glucose donor 1., ${ }^{92}$

Synthesis. We prepared benzylidene-protected glucosazide donors $3^{31}$ and $4^{32}$ with an $O$-benzyl and an $O$-benzoyl, respectively, at C-3, as well as silylidene-protected donor 2, from common building block $12^{33}$ as depicted in Scheme 1 . Hydrolysis of all acetyl esters and the trichloroacetamide was followed by a diazotransfer to install the desired C-2-azide. ${ }^{34}$ Subsequent introduction of the di-tert-butylsilylidene (DTBS) and the benzylidene acetal gave intermediates $13^{31}$ and 14, respectively. Benzylation of $\mathbf{1 4}$ and $\mathbf{1 3}$ and benzoylation of $\mathbf{1 3}$ gave the target donor compounds 2, 3, and 4, respectively. Donor 5 was prepared in two steps from thioglucoside $15^{35}$ by exchange of the phthaloyl group for the DNPY functionality. To this end, compound $\mathbf{1 5}$ was treated with ethylenediamine to give amine 16 , which was treated with DNPY reagent $18^{30,36}$ to furnish the target donor.

Observation of Anomeric Triflates. With these five donors in hand, we investigated the formation of potential covalent reactive intermediates in low-temperature NMR studies. ${ }^{37}$ The donors were treated with the diphenyl sulfoxide/triflic anhydride $\left(\mathrm{Ph}_{2} \mathrm{SO} / \mathrm{Tf}_{2} \mathrm{O}\right)^{38}$ combination of reagents in deuterated dichloromethane. Figure 2 shows the results of these studies, and Table 1 summarizes the anomeric chemical shifts of the observed triflates and the temperatures at which decomposition starts $\left(T_{\text {decomp }}\right)$ [see also Supporting Information (SI)]. Activation of reference donor 1 led to the formation of two species: In addition to the anomeric triflate $19,{ }^{9}$ the oxosulfonium triflate $19 \boldsymbol{\alpha}^{*}(6.68 \mathrm{ppm}, 3.6 \mathrm{~Hz})$ was also formed, as was confirmed by the activation of a sample containing additional $\mathrm{Ph}_{2} \mathrm{SO}$ (see SI). Donors 2 and 3 were
Scheme 1. Preparation of Donors $2-5^{a}$

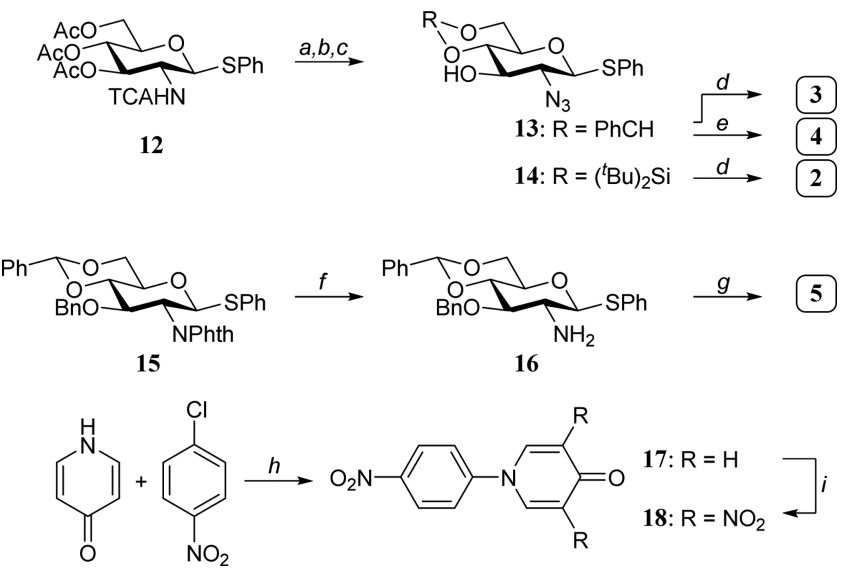

${ }^{a}$ Reagents and conditions: (a) (i) $\mathrm{K}_{2} \mathrm{CO}_{3}, \mathrm{EtOH}, \mathrm{H}_{2} \mathrm{O}$; (ii) $\mathrm{CuSO}_{4}$. $5 \mathrm{H}_{2} \mathrm{O}$, imidazole-1-sulfonyl azide hydrochloride; ${ }^{34}$ (b) di-tert-butylsilyl bis(trifluoromethanesulfonate), pyridine, 14 [71\% (three steps)]; (c) $\mathrm{PhCH}(\mathrm{OMe})_{2}, p-\mathrm{TsOH} \cdot \mathrm{H}_{2} \mathrm{O}, 13$ [78\% (three steps)]; (d) $\mathrm{BnBr}$, $\mathrm{NaH}, \mathrm{DMF}, 2$ (80\%), 3 (89\%); (e) BzCl, DMAP, pyridine, DCM (90\%); (f) ethylenediamine, EtOH (88\%); (g) 18, AcOH/pyridine $(1: 16, \mathrm{v} / \mathrm{v})(98 \%) ;(\mathrm{h}) \mathrm{K}_{2} \mathrm{CO}_{3}, \mathrm{NMP}(85 \%) ;(\mathrm{i}) \mathrm{HNO}_{3}, \mathrm{H}_{2} \mathrm{SO}_{4}$ $(60 \%)$.

cleanly converted to their anomeric $\alpha$-triflates 20 and 21, respectively, by treatment with the activation couple at $-80^{\circ} \mathrm{C}$. Activation of donor 4 proceeded more slowly, and an increase of the temperature from -80 to $-35{ }^{\circ} \mathrm{C}$ was required for complete activation. Donor 5 proved difficult to study by lowtemperature NMR spectroscopy because of significant line broadening in the resonance sets for both the donor and the products formed upon activation. Complete activation of the thioglycoside could only be achieved at $-40{ }^{\circ} \mathrm{C}$, but at this temperature, decomposition of the reactive intermediates also set in. Two anomeric signals can be discriminated in the spectrum of the activated DNPY donor 5 (Figure 2), and we assign these as the intermediate triflate $(6.06 \mathrm{ppm})$ and oxosulfonium triflate $(6.54 \mathrm{ppm})$. Unfortunately, complete characterization was hampered by the severe line broadening. ${ }^{39}$ The reactive intermediates formed all decomposed to give the glucal product 24 . The formation of the glucal double bond is relatively fast, as the proton at $\mathrm{C}-2$ is readily eliminated to provide the enol ether double bond that is conjugated to the DNPY aromatic ring.

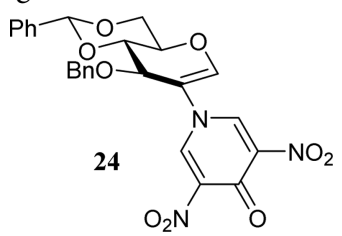

Competitive Glycosylations and Relative Reactivities. To investigate the reactivities of donors 1-5, a series of competitive glycosylations were performed between the different thioglycosides. ${ }^{40-44}$ In these competition experiments, we used an in situ activation protocol, employing $\mathrm{N}$ iodosuccinimide (NIS)/trifluoromethanesulfonic acid (TfOH) as the activator and 2,3,4-tri-O-benzyl- $\alpha$-O-methyl glucose (25) as the acceptor, as is commonly done to determine the reactivities of thioglycoside donors. ${ }^{45,46}$ It should be noted, however, that the reactivity of the thiophenyl donor does not directly compare with the reactivity of an intermediate triflate in 


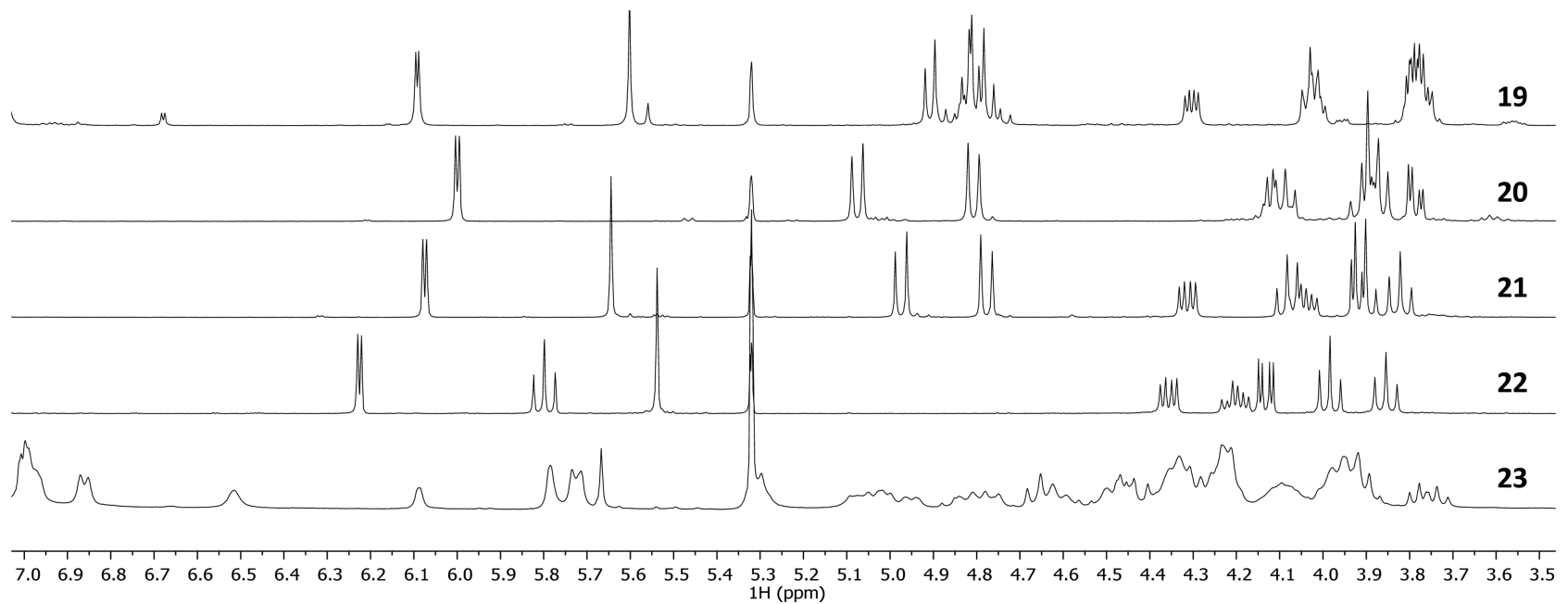

Figure 2. ${ }^{1} \mathrm{H}$ NMR spectra of activated donors 1-5 showing their respective anomeric triflates 19-23.

Table 1. Anomeric Triflates Observed

\begin{tabular}{|c|c|c|c|c|}
\hline Entry & Triflate & $\begin{array}{c}{ }^{1} \mathrm{H} \delta \\
(\mathrm{ppm}) \\
{ }^{3} J_{\mathrm{H} 1-\mathrm{H} 2}{ }^{a} \\
\end{array}$ & $\begin{array}{l}{ }^{13} \mathrm{C} \delta \\
(\mathrm{ppm})\end{array}$ & $\begin{array}{l}\mathrm{T}_{\text {decomp }} \\
\left({ }^{\circ} \mathrm{C}\right)\end{array}$ \\
\hline 1 & & $\begin{array}{l}6.09 \\
3.4 \mathrm{~Hz}\end{array}$ & 106.1 & -20 \\
\hline 2 & & $\begin{array}{l}6.00 \\
3.4 \mathrm{~Hz}\end{array}$ & 104.8 & -30 \\
\hline 3 & & $\begin{array}{l}6.07 \\
3.5 \mathrm{~Hz}\end{array}$ & 105.0 & -20 \\
\hline 4 & & $\begin{array}{l}6.23 \\
3.5 \mathrm{~Hz}\end{array}$ & 104.5 & -10 \\
\hline 5 & 23 & 6.06 & 102.2 & -40 \\
\hline
\end{tabular}

the glycosylation, although it does provide an indication of the relative disarming or arming nature of the protecting groups present on the different donors. It is apparent from Table 2 that the azide has a profound effect on the reactivity of donor 3 , as it is completely outcompeted by the C-2-O-benzyl donor $\mathbf{1}^{46}$ Silylidene donor $\mathbf{2}$ is more reactive than donor 3 , and the disaccharide products derived from donors 2 and 3 are formed in a 6:1 ratio. C-3-O-Benzyl donor 3 in turn outcompetes benzoylated donor 4 slightly, as a result of the electronwithdrawing nature of the benzoate, giving a 1.6:1 ratio of the addition products $3 \mathrm{C}$ and $4 \mathrm{C}^{47-49}$ DNPY-protected donor $\mathbf{5}$ is the least reactive of the set of donors, as it did not provide any disaccharide product in the competition experiment with donor 4.

Glycosylations. With the reactivities of these five donors established, the series of glycosylations with model acceptors
Table 2. Competitive Donor Activations

\begin{tabular}{|c|c|c|c|c|}
\hline \multirow[t]{2}{*}{$\begin{array}{c}\text { Donor I } \\
1 \text { eq. }\end{array}+$} & \multirow[t]{2}{*}{$\begin{array}{c}\text { Donor II } \\
1 \text { eq. }\end{array}$} & \multirow{2}{*}{$252 \mathrm{eq}}$. & \multirow{2}{*}{$\begin{array}{l}\begin{array}{c}1 \text { eq. NIS } \\
0.1 \text { eq. TfOH }\end{array} \\
\stackrel{\begin{array}{c}\text { DCM }(0.05 \mathrm{M}) \\
3 \AA \text { M.S. }\end{array}}{-40^{\circ} \mathrm{C} \text { to } 0^{\circ} \mathrm{C}, 3 \mathrm{~h}}\end{array}$} & \multirow{2}{*}{$\begin{array}{l}\text { disaccharide } \\
\text { product ratio }\end{array}$} \\
\hline & & & & \\
\hline entry & donor I & donor II & products $^{a}$ & yield $^{b}(\%)$ \\
\hline 1 & 1 & 2 & $1 C / 2 C, 14: 1$ & 65 \\
\hline 2 & 1 & 3 & $1 \mathrm{C} / 3 \mathrm{C}, 1: 0$ & 80 \\
\hline 3 & 2 & 3 & $2 \mathrm{C} / 3 \mathrm{C}, 6: 1$ & 37 \\
\hline 4 & 3 & 4 & $3 \mathrm{C} / 4 \mathrm{C}, 1.6: 1$ & 39 \\
\hline $5^{c}$ & 4 & 5 & $4 \mathrm{C} / 5 \mathrm{C}, 1: 0$ & 64 \\
\hline
\end{tabular}

${ }^{a}$ Determined by ${ }^{1} \mathrm{H}$ NMR spectroscopy of the isolated disaccharide. ${ }^{b}$ The disaccharide fraction was quantified after isolation by sizeexclusion chromatography and related to the limiting amount of NIS (see Experimental Section). ${ }^{c}$ The combined donor concentration was $0.1 \mathrm{M}$, triflic acid was added at $-20{ }^{\circ} \mathrm{C}$, the reaction mixture was heated to $+15{ }^{\circ} \mathrm{C}$ overnight, and then the reaction was quenched $\left(\mathrm{Et}_{3} \mathrm{~N}\right)$.

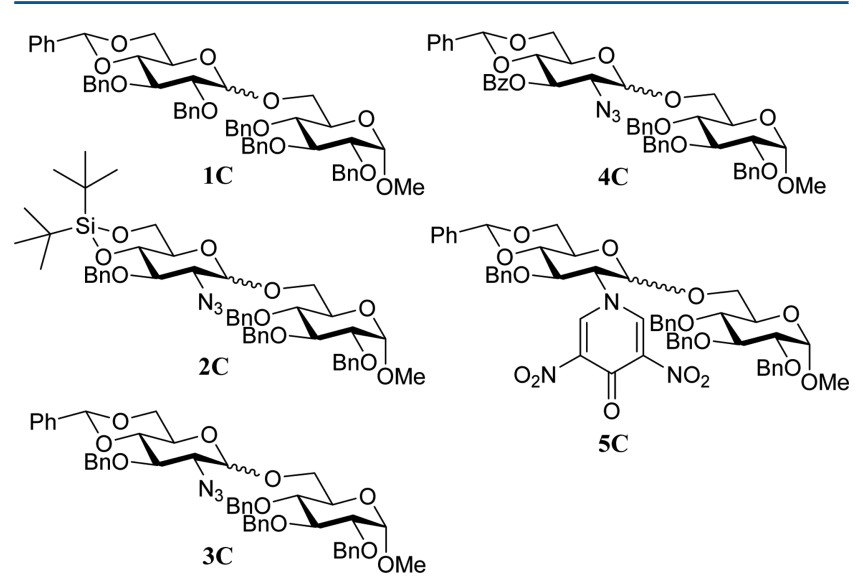

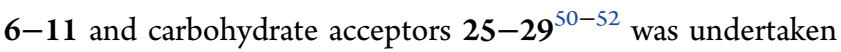
using the $\mathrm{Ph}_{2} \mathrm{SO} / \mathrm{Tf}_{2} \mathrm{O}$ preactivation procedure. Table 3 list all glycosylations ordered by acceptor and donor reactivities. A clear relationship between the acceptor nucleophilicity and the stereochemical outcome of the glycosylation reactions of all studied glucosamine donors was observed, in line with the results previously obtained with donor 1 . Upon comparison of the outcomes of the coupling reactions of glucosazide 3 with 
Table 3. Glycosylations of Donors 1-5 with Model Acceptors 6-11 and Carbohydrate Acceptors 25-29

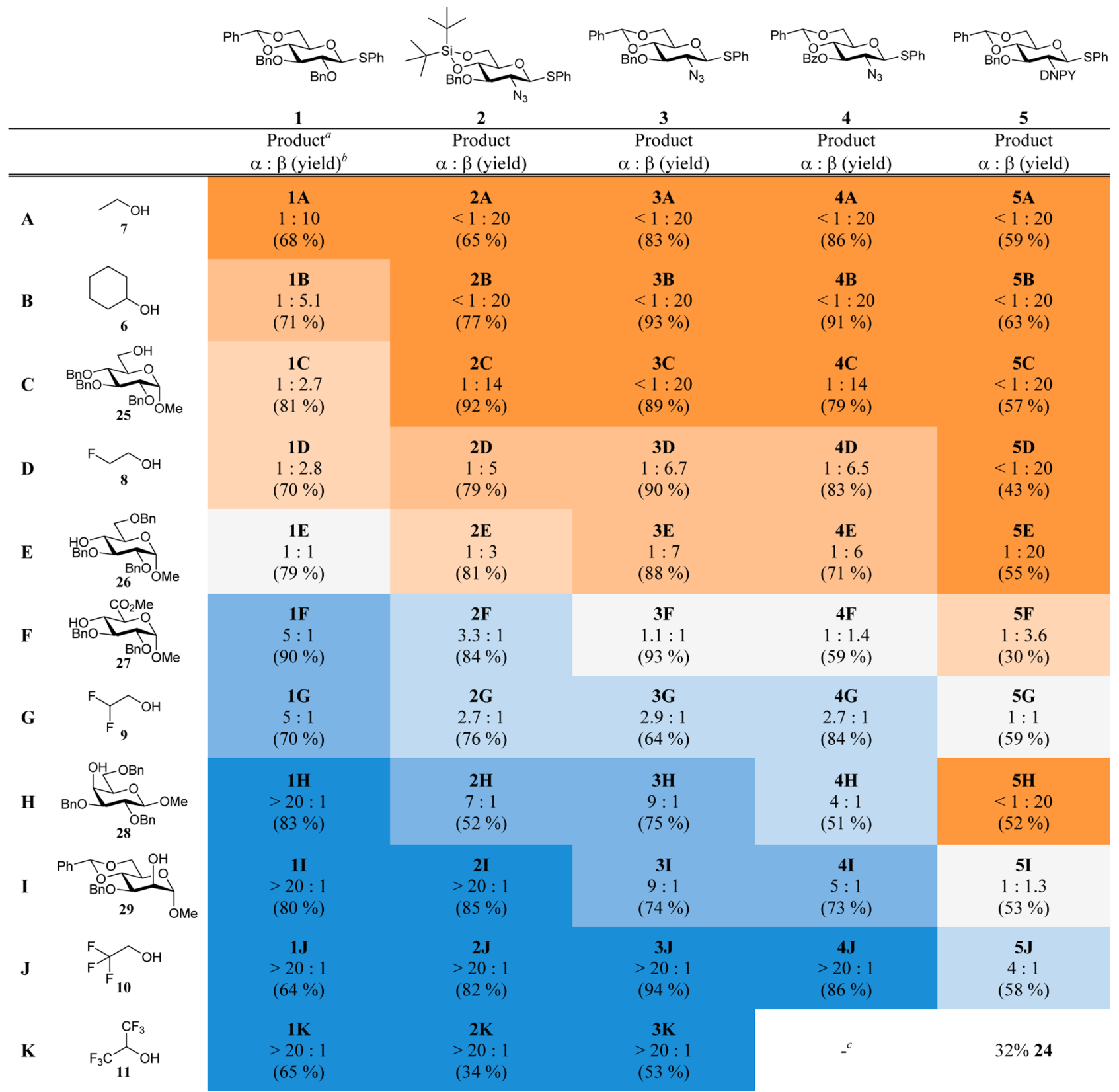

${ }^{a}$ Glycosylation results for donor $\mathbf{1}$ reported previously by van der Vorm et al. ${ }^{22}{ }^{b}$ Ratio and yield of isolated product after column chromatography; anomers were not separated. ${ }^{c}$ Only the hydrolyzed donor was found.

the results obtained using C-2-O-benzyl donor $\mathbf{1}$, it becomes apparent that the latter donor reacts with higher $\alpha$-selectivity. Donor 2, bearing the DTBS group, overall provides slightly more of the $\alpha$-linked products than its benzylidene counterpart 3. The stereoselectivity of the condensations of donor 4, bearing an additional electron-withdrawing protecting group (i.e., the C-3-O-benzoyl), is very similar to the stereoselectivity observed with C-3-O-benzyl donor 3. Finally, donor 5, carrying the strongly electron-withdrawing DNPY group, is the most $\beta$ selective of the series of donors listed in Table $3 .^{53}$

The selectivities of glycosylations with carbohydrate acceptors were also found to vary in a nucleophilicity-dependent fashion. The primary perbenzylated acceptor 25 reacted similarly to ethanol 7 to give primarily the $\beta$-linked products for all glucosamine donors studied. Secondary carbohydrate acceptors that were less nucleophilic showed variations in selectivity with the proportion of $\alpha$-product increasing with decreasing acceptor reactivity. In line with our previous studies, the nucleophilicities of the secondary equatorial carbohydrate alcohols fall somewhere between the reactivities of MFE and DFE, with the reactivities of the axial hydroxyls approaching the reactivity of TFE. The differences in the reactivities of the donors are reflected in the stereoselectivities of both the glycosylations that involve the model acceptors and the glycosylations with the carbohydrate acceptors. A recurring trend is apparent for all acceptors, with the most reactive donor 1 providing the most $\alpha$-linked productand the least reactive donor $\mathbf{5}$ giving the least $\alpha$-linked product.

Mechanistic Discussion. Two major trends become apparent from the table of glycosylations (Table 3). First, 

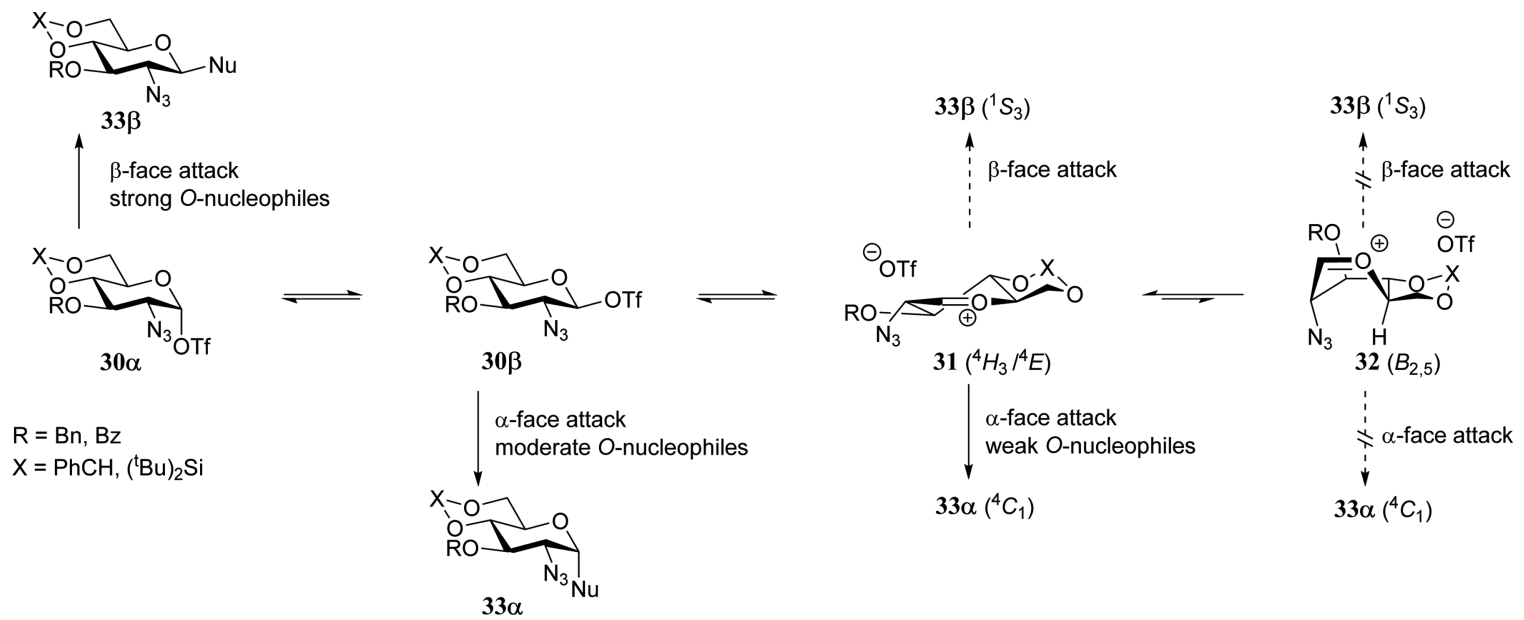

Figure 3. Reactive intermediates and reaction pathways for the 4,6-tethered glucosazide donors.

with decreasing acceptor nucleophilicity, the $\alpha / \beta$ ratio increases. Second, decreasing donor reactivity corresponds to a decrease in the $\alpha / \beta$ ratio. These trends also emerged during our previous studies involving benzylidene glucose, mannose, mannuronic acid, and fucosazide donors. ${ }^{22,28}$ The reactive intermediates that can play a role in the glycosylations of the conformationally restricted glucosamine donors and the reaction trajectories of the incoming nucleophiles are presented in Figure 3. Previous studies by the group of Crich have indicated that substitutions on the benzylidene glucosyl triflate 19 proceed in an $\mathrm{S}_{\mathrm{N}} 2$-like manner. In those mechanistic studies, which involved the determination of kinetic isotope effects and cation-clock methodology, isopropanol was used as an acceptor. ${ }^{12,14}$ In the kinetic scenario that was proposed, the relatively stable $\alpha$-trilfate (observed by low-temperature NMR spectroscopy) is in equilibrium with its more reactive $\beta$ counterpart. In both species, the triflate can be displaced by alcohols if they are nucleophilic enough. The higher $\beta$ selectivity that is seen for the glucosazide and DNPYglucosamine donors in comparison to donor $\mathbf{1}$ can be explained by the stronger electron-withdrawing effect of the azide with respect to the benzyl ether. This leads to a more stable covalent $\alpha$-triflate and favors an associative displacement mechanism. A similar effect was observed by the group of Crich in glycosylations of the analogous 2-deoxy-2-fluoro benzylidene glucosides. $^{54}$ The DNPY group is even more electronwithdrawing, leading to a further increase in $\beta$-selectivity through associative displacement. However, an $S_{N}$ 2-like reaction pathway is less likely for the weaker nucleophiles, such as TFE and HFIP. The high $\alpha$-selectivity for these acceptors can be explained perhaps more precisely by considering the involvement of more electrophilic intermediates such as the glycosyl oxocarbenium ion. The benzylidene and silylidene protecting groups restrict the conformational space that the donor pyranosides can adopt, and the intermediate oxocarbenium ion likely adopt a ${ }^{3} \mathrm{E} /{ }^{3} \mathrm{H}_{4}$-like conformation. ${ }^{55,56}$ Nucleophiles attack this envelope/half-chair conformer preferentially from the bottom face to lead to the $\alpha$ linked prodcuts through a chairlike transition state. ${ }^{57}$ The more reactive donors more readily dissociate to form an oxocarbenium ion, and this accounts for the increased $\alpha$-selectivity for these donors. Donor 2, bearing the silylidene group is the most reactive of the studied glucosamine donors. It also is slightly more flexible than the benzylidene restricted donors, and these two factors allow the activated donor to more readily form a flattened oxocarbenium ion-like intermediate. Consequently, it is the most $\alpha$-selective of the studied glucosamine donors. Finally, it is notable that the C-3-O-benzoyl-protected glucosazide 4 reacts in a slightly more $\beta$-selective fashion than its C-3-O-benzyl counterpart 3. In light of the discussion above, this makes sense, as the electron-withdrawing benzoyl stabilizes the anomeric $\alpha$-triflate. It contrasts, however, with the behavior of acyl groups at the C-3 position of benzylidene mannosyl donors. The 1,2-cis selectivity generally observed for these donors can be completely changed to selectively give the $\alpha$-linked products by installing a C-3-O-acyl group in the donor. $^{58,59}$ The difference between the benzylidene mannose and benzylidene glucose series can be found in the different geometries that the oxocarbenium ions adopts. For the benzylidene mannose system, a $B_{2,5}$-like structure is one of the lower-energy oxocarbenium ion conformers. ${ }^{12,55,56}$ In this constellation, the C-3-benzoate can fold over to the electrondepleted anomeric center to provide stabilization, without a major skeletal rearrangement. For the benzylidene glucose, on the other hand, a $B_{2,5}$-like structure such as $\mathbf{3 2}$ is significantly less favorable because this puts the $\mathrm{C}$-2-azide in a flagpole position. Given the selectivities observed for this donor, influences arising from this boat conformation do not play a significant role here.

\section{CONCLUSIONS}

A set of model acceptors of gradually changing nucleophilicity has been used to investigate how the stereochemistry of glycosylations involving 4,6-tethered glucosamine donors relates to the nucleophilicity of the acceptor. The set of acceptors was complemented by a suite of carbohydrate alcohols to translate the results obtained with the model acceptors to a more relevant glycosylation setting. Four glucosamine donors were probed that differed in the type of tether spanning the C-4 and C-6 alcohols, the nature of the protecting group at the $\mathrm{C}-3-\mathrm{OH}$, and the nature of the amino functionality at C-2. Similarly to the previously described benzylidene glucose donor $\mathbf{1}$, the stereoselectivity of the studied glucosamine donors show a strong correlation to the nucleophilicity of the acceptor, with strong nucleophiles providing completely $\beta$-selective condensations and weak nucleophiles selectively leading to the formation of the $\alpha$ linked products. Benzylidene glucosazide donors are less $\alpha$ - 
selective than their C-2-O-benzyl congeners, because of the increased electron-withdrawing power of the azide, which retards the formation of an oxocarbenium ion species and favors a more associative mechanistic pathway. We have also introduced a novel protecting group for the C-2-amino group: the dinitropyridone functionality. ${ }^{29,30,36}$ Although this group is easily installed and removed from the $\mathrm{C}$-2-amine, its strongly electron-withdrawing character limits its use. In the 4,6benzylidene glucosamine donor studied here, it disarms the donor glycoside to the extent that it turns into a suboptimal glycosyl donor. A major incentive for the reported study was the good to excellent $\alpha$-selectivity that has previously been reported for benzylidene glucose donor 1. Unfortunately, installation of a 4,6-benzylidene on the analogous glucosazide donors does not provide a reliable donor to affect 1,2-cisselective glycosylations. Only with relatively poor nucleophiles are useful stereoselectivities obtained. Changing the benzylidene for a silylidene group, however, turns the donor into a more reactive glycosylating agent showing improved $\alpha$ selectivity. This donor, attractive because of its fully orthogonal protecting group scheme, might find application in the future assembly of oligosaccharides featuring $\alpha$-glucosamines. Finally, we note that this study provides another illustration of the application of the toolset of partially fluorinated ethanols to efficiently map the reactivity-selectivity relationship of a class of donor glycosides. Implementation of this methodology to investigate novel donor systems will provide broader insights into the different mechanistic pathways at play during glycosylations and eventually generate a complete picture of how to tune both reaction partners to achieve stereoselective glycosylation reactions in a predictable manner.

\section{EXPERIMENTAL SECTION}

General Experimental Procedures. All chemicals were of commercial grade and were used as received unless stated otherwise. Dichloromethane (DCM) was stored over activated $4 \AA$ molecular sieves for at least $24 \mathrm{~h}$ before use. Trifluoromethanesulfonic anhydride $\left(\mathrm{Tf}_{2} \mathrm{O}\right)$ was distilled over $\mathrm{P}_{2} \mathrm{O}_{5}$ and stored at $-20{ }^{\circ} \mathrm{C}$ under a nitrogen atmosphere. Triethylamine $\left(\mathrm{Et}_{3} \mathrm{~N}\right)$ was distilled over $\mathrm{CaH}_{2}$ and stored over $\mathrm{KOH}$ pellets. Overnight temperature control was achieved with an FT902 immersion cooler (Julabo). Flash column chromatography was performed on silica gel $60 \AA(0.04-0.063 \mathrm{~mm}$, Screening Devices B.V.). Size-exclusion chromatography was performed on Sephadex (LH-20, GE Healthcare Life Sciences) by isocratic elution with DCM/ $\mathrm{MeOH}(1: 1, \mathrm{v} / \mathrm{v})$. Thin-layer chromatography (TLC) analysis was conducted on TLC silica gel 60 (Kieselgel $60 \mathrm{~F}_{254}$, Merck) with UV detection $(254 \mathrm{~nm})$ and by spraying with $20 \%$ sulfuric acid in ethanol or by spraying with a solution of $\left(\mathrm{NH}_{4}\right)_{6} \mathrm{Mo}_{7} \mathrm{O}_{24} \cdot \mathrm{H}_{2} \mathrm{O}(25 \mathrm{~g} / \mathrm{L})$ and $\left(\mathrm{NH}_{4}\right)_{4} \mathrm{Ce}\left(\mathrm{SO}_{4}\right)_{4} \cdot{ }_{2} \mathrm{H}_{2} \mathrm{O}(10 \mathrm{~g} / \mathrm{L})$ in $10 \%$ aqueous sulfuric acid followed by charring at $\pm 250{ }^{\circ} \mathrm{C}$. High-resolution mass spectroscopy (HRMS) was performed on a Thermo Finnigan LTQ Orbitrap mass spectrometer equipped with an electrospray ion source in positive-ion mode (source voltage $3.5 \mathrm{kV}$, sheath-gas flow rate 10 , capillary temperature $275^{\circ} \mathrm{C}$ ) with a resolution of $R=60.000$ at $\mathrm{m} / z 400$ (mass range of $150-4000) .{ }^{1} \mathrm{H}$ and ${ }^{13} \mathrm{C}$ NMR spectra were recorded on Bruker AV-400, Bruker DMX-400, and Bruker AV-500 NMR instruments. Chemical shifts $(\delta)$ are given in parts per million (ppm) relative to tetramethylsilane as an internal standard or the residual signal of the deuterated solvent. Coupling constants $(J)$ are given in hertz $(\mathrm{Hz})$. All presented ${ }^{13} \mathrm{C}$-attached proton test (APT) spectra are proton-decoupled. NMR peak assignments were made using correlation spectroscopy (COSY) and heteronuclear singlequantum coherence (HSQC). If necessary, additional nuclear Overhauser enhancement spectroscopy (NOESY), heteronuclear multiple-bond correlation (HMBC), and gated HMBC (HMBCGATED) experiments were used to further elucidate the structure.
The anomeric product ratios were based on careful analysis of the crude reaction mixture and the purified reaction product by integration of representative ${ }^{1} \mathrm{H}$ NMR signals. IR spectra were recorded on a Shimadzu FTIR-8300 IR spectrometer and are reported in wavenumbers $\left(\mathrm{cm}^{-1}\right)$. Specific rotations were measured on a Propol automatic polarimeter or an Anton-Paar MCP-100 modular circular polarimeter at $589 \mathrm{~nm}$ unless otherwise stated.

General Procedure for $\mathrm{Tf}_{2} \mathrm{O} / \mathrm{Ph}_{2} \mathrm{SO}$-Mediated Glycosylations. Donor $(0.1 \mathrm{mmol}), \mathrm{Ph}_{2} \mathrm{SO}$ ( $26 \mathrm{mg}, 0.13 \mathrm{mmol}, 1.3$ equiv), and TTBP $^{60}$ (62 mg, $0.25 \mathrm{mmol}, 2.5$ equiv) were coevaporated twice with dry toluene and dissolved in dry DCM $(2 \mathrm{~mL}, 0.05 \mathrm{M}$ donor). Activated $3 \AA$ molecular sieves (rods, $1 / 16$ in. in size) were added, and the reaction mixture was stirred for $1 \mathrm{~h}$ at room temperature under a nitrogen atmosphere. The solution was cooled to $-78^{\circ} \mathrm{C}$, and $\mathrm{Tf}_{2} \mathrm{O}$ (22 $\mu \mathrm{L}, 0.13 \mathrm{mmol}, 1.3$ equiv) was added. The reaction mixture was allowed to warm to $-60{ }^{\circ} \mathrm{C}$ (donors 1,2 , and 3 ), $-45^{\circ} \mathrm{C}$ (donor 5), and $-35^{\circ} \mathrm{C}$ (donor 4) and then recooled to $-78{ }^{\circ} \mathrm{C}$, after which the acceptor $(0.2 \mathrm{mmol}, 2$ equiv) in DCM $(0.4 \mathrm{~mL}, 0.5 \mathrm{M})$ was added. The reaction mixture was allowed to warm to $-40{ }^{\circ} \mathrm{C}$ in approximately $90 \mathrm{~min}$ and stirred for an additional 0-18 h depending on the acceptor. The reaction was quenched with $\mathrm{Et}_{3} \mathrm{~N}(0.1 \mathrm{~mL}, 0.72$ mmol, 5.5 equiv) at $-40{ }^{\circ} \mathrm{C}$, and the mixture was diluted with DCM. The solution was transferred to a separatory funnel, water was added, the layers were separated, and the water phase was extracted once more with DCM. The combined organic layers were dried over $\mathrm{MgSO}_{4}$, filtered, and concentrated in vacuo. Purification by silica gel flash column chromatography and, when needed, sephadex LH-20 size-exclusion chromatography yielded the glycosylation product as a mixture of anomers.

General Procedure for NIS/TfOH-Mediated Competition Experiments. Donor I ( $0.1 \mathrm{mmol}, 1$ equiv), donor II $(0.1 \mathrm{mmol}, 1$ equiv), and acceptor 25 ( $0.2 \mathrm{mmol}, 2$ equiv) were together coevaporated with dry toluene $(2 \times)$. Dry DCM $(4 \mathrm{~mL}$, donor concentration of $0.05 \mathrm{M}$ ), a Teflon stirring bar, and $3 \AA$ activated molecular sieves (rods, $1 / 16$ in. in size) were added, and the mixture was stirred under a nitrogen atmosphere for $1 \mathrm{~h}$ at room temperature. The mixture was cooled to $-40{ }^{\circ} \mathrm{C}$, NIS $(0.1 \mathrm{mmol}, 1$ equiv) was added. TfOH ( $50 \mu \mathrm{L}$ of a freshly prepared $0.2 \mathrm{M}$ stock solution in dry DCM, 0.1 equiv) was added, and the mixture was allowed to warm to $0{ }^{\circ} \mathrm{C}$ in $3 \mathrm{~h} . \mathrm{Et}_{3} \mathrm{~N}(0.1 \mathrm{~mL})$ was added, and the mixture was diluted with EtOAc, washed with saturated aqueous $\mathrm{NaS}_{2} \mathrm{O}_{3}$ and brine, dried over $\mathrm{Na}_{2} \mathrm{SO}_{4}$, and concentrated in vacuo. Size-exclusion chromatography (Sephadex LH-20, 1:1 DCM/MeOH) enabled isolation of the disaccharide products and the monosaccharide remainders, which were both analyzed by NMR spectroscopy. The yield of the disaccharide fraction was determined. For the competition between donors $\mathbf{4}$ and $\mathbf{5}$, a $0.1 \mathrm{M}$ concentration and a starting temperature of $-20{ }^{\circ} \mathrm{C}$ were used, and the mixture was allowed to warm to $+15^{\circ} \mathrm{C}$ in $18 \mathrm{~h}$.

General Procedure for Low-Temperature NMR Experiments. A mixture of donor $(30 \mu \mathrm{mol})$ and $\mathrm{Ph}_{2} \mathrm{SO}(39 \mu \mathrm{mol})$ was coevaporated with dry toluene twice. [For the activation of donor $\mathbf{1}$, TTBP $(75 \mu \mathrm{mol})$ was also added.] Under a nitrogen atmosphere, $\mathrm{CD}_{2} \mathrm{Cl}_{2}(0.6 \mathrm{~mL})$ was added, and the mixture was transferred to a nitrogen-flushed NMR tube that was then closed with an NMR tube septum. The NMR magnet was cooled to $-80{ }^{\circ} \mathrm{C}$, locked, and shimmed and the sample was measured prior to activation. In a long narrow cold bath $\left(\mathrm{EtOH},-85^{\circ} \mathrm{C}\right)$ the sample was treated with $\mathrm{Tf}_{2} \mathrm{O}$ (39 $\mu \mathrm{mol})$, shaken thrice, and cooled again after each shaking. The cold sample was wiped dry and quickly inserted back in the cold magnet. The first ${ }^{1} \mathrm{H}$ NMR spectrum was immediately recorded. The sample was then reshimmed, and spectra were recorded in $10{ }^{\circ} \mathrm{C}$ intervals with at least $5 \mathrm{~min}$ of equilibration time for every temperature.

Phenyl 2-Azido-4,6-O-benzylidene-2-deoxy-1-thio- $\beta$-D-glucopyranoside (13). To a suspension of thioglycoside $12^{33}(27.14 \mathrm{~g}, 50$ mmol, 1 equiv) in EtOH $(200 \mathrm{~mL})$ were added $\mathrm{K}_{2} \mathrm{CO}_{3}(41.5 \mathrm{~g}, 300$ mmol, 6 equiv) and $20 \mathrm{~mL}$ of $\mathrm{H}_{2} \mathrm{O}$, and the mixture was refluxed overnight. The flask was cooled to room temperature, and to the crude free amine ${ }^{61}$ was added the diazo transfer reagent imidazole-1-sulfonyl azide hydrochloride ${ }^{34}$ (13.10 g, $62.5 \mathrm{mmol}, 1.25$ equiv) in three equal 
portions, followed by a catalytic amount of $\mathrm{CuSO}_{4} \cdot 5 \mathrm{H}_{2} \mathrm{O}(125 \mathrm{mg}$, $0.5 \mathrm{mmol}, 0.01$ equiv). After the solution had been stirred for $5 \mathrm{~h}$, it was filtered and reduced to one-fourth its volume in vacuo. $\mathrm{H}_{2} \mathrm{O}(150$ $\mathrm{mL})$ and $1 \mathrm{M}$ aqueous $\mathrm{HCl}(150 \mathrm{~mL})$ were added to obtain an acidic $(\mathrm{pH} \sim 3)$ solution, which was extracted with EtOAc $(3 \times 120 \mathrm{~mL})$. The combined organic layers were washed with saturated aqueous $\mathrm{NaHCO}_{3}(150 \mathrm{~mL})$ and brine $(150 \mathrm{~mL})$, dried with $\mathrm{MgSO}_{4}$, and concentrated in vacuo to obtain the crude azide phenyl 2-azido-2deoxy-1-thio- $\beta$-D-glucopyranoside. ${ }^{62}$ The crude azide $(\leq 50 \mathrm{mmol})$ was coevaporated with toluene twice and subsequently dissolved in DMF $(50 \mathrm{~mL})$ and $\mathrm{MeCN}(200 \mathrm{~mL})$ to which benzaldehyde dimethyl acetal $\left(15 \mathrm{~mL}, 100 \mathrm{mmol}, 2\right.$ equiv) and $p-\mathrm{TsOH} \cdot \mathrm{H}_{2} \mathrm{O}(950 \mathrm{mg}, 5 \mathrm{mmol}, 0.1$ equiv) had been added. The reaction mixture was heated at $60{ }^{\circ} \mathrm{C}$ overnight and then heated at $60{ }^{\circ} \mathrm{C}$ for an additional $5 \mathrm{~h}$ under reduced pressure $(300 \mathrm{mbar})$ to reduce the volume to one-third. The reaction was quenched by the addition of triethylamine $(1 \mathrm{~mL})$, and the reaction mixture was diluted with EtOAc $(350 \mathrm{~mL})$ and washed with $\mathrm{H}_{2} \mathrm{O}(2 \times 100 \mathrm{~mL})$, saturated aqueous $\mathrm{NaHCO}_{3}(1 \times 100 \mathrm{~mL})$, and brine $(1 \times 100 \mathrm{~mL})$. The organic layer was dried $\left(\mathrm{MgSO}_{4}\right)$ and concentrated in vacuo. The crude mixture was purified by percipitation from hot EtOAc $(100 \mathrm{~mL}) /$ heptane $(300 \mathrm{~mL})$ through the addition of petroleum ether $(500 \mathrm{~mL})$ as the mixture was stirred and slowly cooled to $0{ }^{\circ} \mathrm{C}$ to obtain the title compound as a white powder $(11.38$ g, $29.5 \mathrm{mmol}, 59 \%$ ). The mother liquors were purified by flash column chromatography (8:1 to $4: 1$ pentane/EtOAc) to obtain an additional batch of white solid product $(3.8 \mathrm{~g}, 9.6 \mathrm{mmol}$, total yield $=39.1 \mathrm{mmol}$, $78 \%$, three steps). A purified sample could be recrystallized from either hot $\mathrm{MeOH}$ or EtOAc/petroleum ether to obtain white cotton-like needles. $R_{\mathrm{f}} 0.50$ (6:1 pentane/EtOAc). Spectroscopic data were in accord with those reported previously. ${ }^{31}{ }^{1} \mathrm{H} \mathrm{NMR}\left(\mathrm{CDCl}_{3}, 400 \mathrm{MHz}\right.$, $\mathrm{H}-\mathrm{H}$ COSY, HSQC): $\delta 7.64-7.52\left(\mathrm{~m}, 2 \mathrm{H}, \mathrm{CH}_{\text {arom }}\right), 7.50-7.43(\mathrm{~m}$, $\left.2 \mathrm{H}, \mathrm{CH}_{\text {arom }}\right), 7.42-7.32\left(\mathrm{~m}, 6 \mathrm{H}, \mathrm{CH}_{\text {arom }}\right), 5.53(\mathrm{~s}, 1 \mathrm{H}, \mathrm{CHPh}), 4.54$ $(\mathrm{d}, 1 \mathrm{H}, J=10.1 \mathrm{~Hz}, \mathrm{H}-1), 4.38(\mathrm{dd}, 1 \mathrm{H}, J=10.5,4.6 \mathrm{~Hz}, \mathrm{H}-6), 3.85-$ $3.70(\mathrm{~m}, 2 \mathrm{H}, \mathrm{H}-3, \mathrm{H}-6), 3.52-3.40(\mathrm{~m}, 2 \mathrm{H}, \mathrm{H}-4, \mathrm{H}-5), 3.35$ (dd, $1 \mathrm{H}, J$ $=10.2,9.0 \mathrm{~Hz}, \mathrm{H}-6), 2.75(\mathrm{bs}, 1 \mathrm{H}, 3-\mathrm{OH}) .{ }^{13} \mathrm{C}-\mathrm{APT} \mathrm{NMR}\left(\mathrm{CDCl}_{3}\right.$, $101 \mathrm{MHz}, \mathrm{HSQC}): \delta 136.8\left(\mathrm{C}_{\mathrm{q}} \mathrm{CHPh}\right), 133.8\left(\mathrm{CH}_{\text {arom }}\right), 130.9\left(\mathrm{C}_{\mathrm{q}}\right.$ $\mathrm{SPh}), 129.6,129.3,128.8,128.5,126.4\left(\mathrm{CH}_{\text {arom }}\right), 102.1(\mathrm{CHPh}), 86.9$ (C-1), 80.3 (C-4), 74.2 (C-3), 70.4 (C-5), 68.5 (C-6), 65.2 (C-2). HRMS: $[\mathrm{M}+\mathrm{H}]^{+}$calcd for $\mathrm{C}_{19} \mathrm{H}_{20} \mathrm{~N}_{3} \mathrm{O}_{4} \mathrm{~S}$ 386.11690, found 386.11708 .

Phenyl 2-Azido-2-deoxy-4,6-O-di-tert-butylsilylidene-1-thio- $\beta$-Dglucopyranoside (14). Crude triol phenyl 2 -azido-2-deoxy-1-thio- $\beta$ D-glucopyranoside (synthesized as described for compound 13) $(\leq 10$ mmol) was dissolved in pyridine $(15 \mathrm{~mL})$ and cooled to $0{ }^{\circ} \mathrm{C}$. Di-tertbutylsilyl bis(trifluoromethanesulfonate) $(3.6 \mathrm{~mL}, 11 \mathrm{mmol}, 1.1$ equiv) was slowly added, and the mixture was stirred for $1 \mathrm{~h}$ before the reaction was quenched with $\mathrm{MeOH}$. The reaction mixture was diluted with $200 \mathrm{~mL}$ of $\mathrm{Et}_{2} \mathrm{O}$ and washed with $1 \mathrm{M}$ aqueous $\mathrm{HCl}(3 \times 60 \mathrm{~mL})$, saturated aqueous $\mathrm{NaHCO}_{3}(60 \mathrm{~mL})$, and brine $(60 \mathrm{~mL})$. The organic layer was dried with $\mathrm{Na}_{2} \mathrm{SO}_{4}$, filtered, and concentrated in vacuo. Purification by flash column chromatography (1-10\% $\mathrm{Et}_{2} \mathrm{O} /$ pentane) afforded the silylidene-protected title compound as a colorless oil (3.10 g, $7.1 \mathrm{mmol}, 71 \%$ over three steps). $R_{\mathrm{f}} 0.18$ (19:1 pentane/ $\left.\mathrm{Et}_{2} \mathrm{O}\right) .[\alpha]_{\mathrm{D}}^{23}=-42.6^{\circ}\left(c=1.0, \mathrm{CHCl}_{3}\right)$. IR (neat): 652, 733, 824, $1072,1092,1155,1277,1474,2112,2859,2884,2934,2963,3449 .{ }^{1} \mathrm{H}$ NMR $\left(\mathrm{CDCl}_{3}, 400 \mathrm{MHz}, \mathrm{H}-\mathrm{H}\right.$ COSY, HSQC) $\delta 7.57-7.51(\mathrm{~m}, 2 \mathrm{H}$, $\left.\mathrm{CH}_{\text {arom }}\right), 7.36-7.31\left(\mathrm{~m}, 3 \mathrm{H}, \mathrm{CH}_{\text {arom }}\right), 4.49(\mathrm{~d}, 1 \mathrm{H}, J=10.2 \mathrm{~Hz}, \mathrm{H}-1)$, 4.21 (dd, $1 \mathrm{H}, J=10.2,5.1 \mathrm{~Hz}, \mathrm{H}-6), 3.89(\mathrm{t}, 1 \mathrm{H}, J=10.2 \mathrm{~Hz}, \mathrm{H}-6)$, $3.64(\mathrm{t}, 1 \mathrm{H}, J=9.1 \mathrm{~Hz}, \mathrm{H}-4), 3.56(\mathrm{td}, 1 \mathrm{H}, J=9.0,1.2 \mathrm{~Hz}, \mathrm{H}-3), 3.40$ (ddd, $1 \mathrm{H}, J=10.1,9.3,5.1 \mathrm{~Hz}, \mathrm{H}-5), 3.31(\mathrm{dd}, 1 \mathrm{H}, J=10.2,9.1 \mathrm{~Hz}, \mathrm{H}-$ 2), $2.92(\mathrm{~d}, 1 \mathrm{H}, J=1.6 \mathrm{~Hz}, 3-\mathrm{OH}), 1.04\left(\mathrm{~s}, 9 \mathrm{H}, \mathrm{CH}_{3}{ }^{\mathrm{t}} \mathrm{Bu}\right), 0.97(\mathrm{~s}, 9 \mathrm{H}$, $\left.\mathrm{CH}_{3}{ }^{\mathrm{t}} \mathrm{Bu}\right) .{ }^{13} \mathrm{C}-\mathrm{APT}$ NMR $\left(\mathrm{CDCl}_{3}, 101 \mathrm{MHz}, \mathrm{HSQC}\right): \delta 133.7$ $\left(\mathrm{CH}_{\text {arom }}\right), 131.2\left(\mathrm{C}_{\mathrm{q}}\right), 129.2,128.7\left(\mathrm{CH}_{\text {arom }}\right), 86.8(\mathrm{C}-1), 77.4(\mathrm{C}-3)$, 76.6 (C-4), 74.4 (C-5), 66.0 (C-6), $64.4(\mathrm{C}-2), 27.5,27.0\left(\mathrm{CH}_{3}{ }^{\mathrm{t}} \mathrm{Bu}\right)$, 22.8, $20.0\left(\mathrm{C}_{\mathrm{q}}{ }^{\mathrm{t}} \mathrm{Bu}\right)$. HRMS: $\left[\mathrm{M}-\mathrm{N}_{2}+\mathrm{H}\right]^{+}$calcd for $\mathrm{C}_{20} \mathrm{H}_{32} \mathrm{NO}_{4} \mathrm{SSi}$ 410.18213, found 410.18220.

Phenyl 2-Azido-3-O-benzyl-2-deoxy-4,6-O-di-tert-butylsilylidene1-thio- $\beta$-D-glucopyranoside (2). Compound $14(1.4 \mathrm{~g}, 3.2 \mathrm{mmol}$ ) was dissolved in DMF (15 mL) and cooled to $0{ }^{\circ} \mathrm{C}$. Benzyl bromide (421 $\mu \mathrm{L}, 3.52 \mathrm{mmol}, 1.1$ equiv) and $\mathrm{NaH}(60 \%$ dispersion in mineral oil,
$166 \mathrm{mg}, 4.16 \mathrm{mmol}, 1.3$ equiv) were added, and the reaction mixture was stirred for $2 \mathrm{~h}$ at $0{ }^{\circ} \mathrm{C}$ and then for $1 \mathrm{~h}$ at room temperature. The reaction was quenched with $\mathrm{MeOH}$, and $\mathrm{H}_{2} \mathrm{O}(100 \mathrm{~mL})$ was added. The water phase was extracted three times with $30 \mathrm{~mL}$ of $\mathrm{Et}_{2} \mathrm{O}$, and the combined organic layers were washed with brine $(2 \times)$, dried with $\mathrm{Na}_{2} \mathrm{SO}_{4}$, and concentrated under reduced pressure. Purification by flash column chromatography $\left(1 \%-8 \% \quad \mathrm{Et}_{2} \mathrm{O} /\right.$ pentane $)$ yielded compound 2 as a colorless oil $(1.35 \mathrm{~g}, 2.56 \mathrm{mmol}, 80 \%)$. Additional impurities as observed by ${ }^{1} \mathrm{H}$ NMR spectroscopy originating from the previous crude steps could be removed by size-exclusion chromatography (Sephadex LH-20, 1:1 DCM/MeOH). $R_{\mathrm{f}} 0.51$ (19:1 pentane/ $\left.\mathrm{Et}_{2} \mathrm{O}\right) .[\alpha]_{\mathrm{D}}^{23}=-85.0^{\circ}\left(c=1.0, \mathrm{CHCl}_{3}\right)$. IR (neat): 654, 694, 746, 766, $826,1059,1078,1099,1159,1474,2110,2859,2884,2934,2963 .{ }^{1} \mathrm{H}$ NMR $\left(\mathrm{CDCl}_{3}, 400 \mathrm{MHz}, \mathrm{H}-\mathrm{H}\right.$ COSY, HSQC): $\delta 7.55-7.48(\mathrm{~m}, 2 \mathrm{H}$, $\mathrm{CH}_{\text {arom }}$ ), 7.43-7.37 (m, 2H, $\left.\mathrm{CH}_{\text {arom }}\right)$, 7.36-7.27 (m, 6H, $\left.\mathrm{CH}_{\text {arom }}\right)$, $4.99(\mathrm{~d}, 1 \mathrm{H}, J=10.7 \mathrm{~Hz}, \mathrm{CHH} \mathrm{Bn}), 4.81(\mathrm{~d}, 1 \mathrm{H}, J=10.7 \mathrm{~Hz}, \mathrm{CHH}$ $\mathrm{Bn}), 4.41(\mathrm{~d}, 1 \mathrm{H}, J=10.2 \mathrm{~Hz}, \mathrm{H}-1), 4.21(\mathrm{dd}, 1 \mathrm{H}, J=10.3,5.1 \mathrm{~Hz}, \mathrm{H}-$ 6), $3.90(\mathrm{t}, 1 \mathrm{H}, J=10.2 \mathrm{~Hz}, \mathrm{H}-6), 3.87$ (dd, $1 \mathrm{H}, J=9.5,8.7 \mathrm{~Hz}, \mathrm{H}-4)$, $3.48-3.38$ (m, 2H, H-3, H-5), 3.28 (dd, $1 \mathrm{H}, J=10.2,9.2 \mathrm{~Hz}, \mathrm{H}-2)$, $1.07\left(\mathrm{~s}, 9 \mathrm{H}, \mathrm{CH}_{3}{ }^{\mathrm{t}} \mathrm{Bu}\right), 1.01\left(\mathrm{~s}, 9 \mathrm{H}, \mathrm{CH}_{3}{ }^{\mathrm{t}} \mathrm{Bu}\right) .{ }^{13} \mathrm{C}-\mathrm{APT} \operatorname{NMR}\left(\mathrm{CDCl}_{3}\right.$, $101 \mathrm{MHz}, \mathrm{HSQC}): \delta 137.9\left(\mathrm{C}_{\mathrm{q}} \mathrm{Bn}\right), 133.9\left(\mathrm{CH}_{\text {arom }}\right), 130.9\left(\mathrm{C}_{\mathrm{q}} \mathrm{SPh}\right)$, $129.1,128.7,128.5,128.5,128.1\left(\mathrm{CH}_{\text {arom }}\right), 86.4(\mathrm{C}-1), 84.2(\mathrm{C}-3)$, 77.8 (C-4), $75.7\left(\mathrm{CH}_{2} \mathrm{Bn}\right), 74.7$ (C-5), 66.2 (C-6), 64.2 (C-2), 27.5, 27.1 $\left(\mathrm{CH}_{3}{ }^{\mathrm{t}} \mathrm{Bu}\right), 22.7,20.0\left(\mathrm{CH}_{3}{ }^{\mathrm{t}} \mathrm{Bu}\right)$. HRMS: $[\mathrm{M}+\mathrm{H}]^{+}$calcd for $\mathrm{C}_{27} \mathrm{H}_{38} \mathrm{~N}_{3} \mathrm{O}_{4} \mathrm{SSi}$ 528.23468, found 528.23451; $\left[\mathrm{M}-\mathrm{N}_{2}+\mathrm{H}\right]^{+}$calcd for $\mathrm{C}_{27} \mathrm{H}_{38} \mathrm{NO}_{4} \mathrm{SSi}$ 500.22853, found 500.22839.

Phenyl 2-Azido-3-O-benzyl-4,6-O-benzylidene-2-deoxy-1-thio- $\beta$ D-glucopyranoside (3). Compound $13(4.36 \mathrm{~g}, 11.3 \mathrm{mmol}$ ) was coevaporated once with dry toluene and then dissolved in DMF (50 $\mathrm{mL}$ ) and cooled to $0{ }^{\circ} \mathrm{C}$. Benzyl bromide $(1.9 \mathrm{~mL}, 15.8 \mathrm{mmol}, 1.4$ equiv) and $\mathrm{NaH}$ (60\% dispersion in mineral oil, $900 \mathrm{mg}, 22.6 \mathrm{mmol}, 2$ equiv) were added in succession, and the reaction mixture was stirred at room temperature for $4.5 \mathrm{~h}$. $\mathrm{MeOH}(5 \mathrm{~mL})$ was slowly added, and the reaction mixture was diluted with EtOAc $(150 \mathrm{~mL})$ and washed with $\mathrm{H}_{2} \mathrm{O}(2 \times 60 \mathrm{~mL})$ and brine $(50 \mathrm{~mL})$. The organic layer was dried $\left(\mathrm{MgSO}_{4}\right)$, filtered, and concentrated in vacuo. The crude product was purified by crystallization $(10 \mathrm{~mL}$ of hot EtOAc, addition of $100 \mathrm{~mL}$ of petroleum ether) to yield the title compound as a white cotton-like solid $(4.79 \mathrm{~g}, 10.1 \mathrm{mmol}, 89 \%) . R_{\mathrm{f}} 0.71$ (8:1 pentane/ EtOAc). Spectroscopic data were in accord with those reported previously. ${ }^{1} \mathrm{H}$ NMR $\left(\mathrm{CDCl}_{3}, 400 \mathrm{MHz}, \mathrm{H}-\mathrm{H}\right.$ COSY, HSQC): $\delta$ $7.56\left(\mathrm{ddt}, 2 \mathrm{H}, J=5.0,3.4,1.5 \mathrm{~Hz}, \mathrm{CH}_{\text {arom }}\right), 7.47$ (dd, $2 \mathrm{H}, J=7.5,2.3$ $\left.\mathrm{Hz}, \mathrm{CH}_{\text {arom }}\right), 7.42-7.26\left(\mathrm{~m}, 11 \mathrm{H}, \mathrm{CH}_{\text {arom }}\right), 5.57(\mathrm{~s}, 1 \mathrm{H}, \mathrm{CHPh}), 4.91$ $(\mathrm{d}, 1 \mathrm{H}, J=10.9 \mathrm{~Hz}, \mathrm{CHH} \mathrm{Bn}), 4.78(\mathrm{~d}, 1 \mathrm{H}, J=10.9 \mathrm{~Hz}, \mathrm{CHH} \mathrm{Bn})$, 4.49 (d, $1 \mathrm{H}, J=10.2 \mathrm{~Hz}, \mathrm{H}-1$ ), 4.39 (dd, $1 \mathrm{H}, J=10.6,5.0 \mathrm{~Hz}, \mathrm{H}-6)$, $3.79(\mathrm{t}, 1 \mathrm{H}, J=10.3 \mathrm{~Hz}, \mathrm{H}-6), 3.71-3.59(\mathrm{~m}, 2 \mathrm{H}, \mathrm{H}-3, \mathrm{H}-4), 3.52-$ $3.42(\mathrm{~m}, 1 \mathrm{H}, \mathrm{H}-5), 3.41-3.32(\mathrm{~m}, 1 \mathrm{H}, \mathrm{H}-2) .{ }^{13} \mathrm{C}-\mathrm{APT} \mathrm{NMR}\left(\mathrm{CDCl}_{3}\right.$, $101 \mathrm{MHz}, \mathrm{HSQC}): \delta 137.6,137.1\left(\mathrm{C}_{\mathrm{q}}\right), 134.0\left(\mathrm{CH}_{\text {arom }}\right), 130.6\left(\mathrm{C}_{\mathrm{q}}\right.$ $\mathrm{SPh}), 129.2,129.2,128.8,128.5,128.4,128.4,128.1,126.0\left(\mathrm{CH}_{\text {arom }}\right)$, 101.3 (CHPh), 86.5 (C-1), 81.3, 81.0 (C-3, C-4), $75.3\left(\mathrm{CH}_{2} \mathrm{Bn}\right), 70.5$ (C-5), 68.5 (C-6), 64.6 (C-2). HRMS: $[\mathrm{M}+\mathrm{H}]^{+}$calcd for $\mathrm{C}_{26} \mathrm{H}_{26} \mathrm{~N}_{3} \mathrm{O}_{4} \mathrm{~S}$ 476.16385, found 476.16375.

Phenyl 2-Azido-3-O-benzoyl-4,6-O-benzylidene-2-deoxy-1-thio$\beta$-D-glucopyranoside (4). To a $0{ }^{\circ} \mathrm{C}$ solution of compound 13 $(1.34 \mathrm{~g}, 3.48 \mathrm{mmol})$ in DCM $(17 \mathrm{~mL})$ and pyridine $(1.4 \mathrm{~mL}, 34.8$ mmol, 5 equiv) were added benzoyl chloride $(0.61 \mathrm{~mL}, 5.22 \mathrm{mmol}, 1.5$ equiv) and DMAP ( $42 \mathrm{mg}, 0.35 \mathrm{mmol}, 0.1$ equiv). The reaction mixture was allowed to stir overnight, after which $\mathrm{H}_{2} \mathrm{O}$ and DCM were added. The organic layer was separated and washed with saturated aqueous $\mathrm{NaHCO}_{3}$ and brine. The organic layer was dried with $\mathrm{MgSO}_{4}$ and concentrated in vacuo. Flash column chromatography (19:1 to 8:1 pentane/EtOAc) afforded the title compound as a white solid (1.54 g, $3.15 \mathrm{mmol}, 90 \%)$. The product could be recrystallized from EtOAc and petroleum ether to yield a fluffy white solid. $R_{\mathrm{f}} 0.53$ (8:1 pentane/ EtOAc). Spectroscopic data were in accord with those reported previously. ${ }^{6}{ }^{1} \mathrm{H}$ NMR $\left(\mathrm{CDCl}_{3}, 400 \mathrm{MHz}, \mathrm{H}-\mathrm{H}\right.$ COSY, HSQC): $\delta$ $8.05\left(\mathrm{~d}, 2 \mathrm{H}, J=7.3 \mathrm{~Hz}, \mathrm{CH}_{\text {arom }}\right), 7.59(\mathrm{dd}, 2 \mathrm{H}, J=6.5,3.1 \mathrm{~Hz}$, $\left.\mathrm{CH}_{\text {arom }}\right), 7.53\left(\mathrm{t}, 1 \mathrm{H}, J=7.4 \mathrm{~Hz}, \mathrm{CH}_{\text {arom }}\right), 7.44-7.33\left(\mathrm{~m}, 7 \mathrm{H}, \mathrm{CH}_{\text {arom }}\right)$, 7.29-7.23 (m, $\left.3 \mathrm{H}, \mathrm{CH}_{\text {arom }}\right), 5.52(\mathrm{t}, 1 \mathrm{H}, J=9.6 \mathrm{~Hz}, \mathrm{H}-3), 5.46(\mathrm{~s}, 1 \mathrm{H}$, CHPh), 4.69 (d, 1H, J = 10.1 Hz, H-1), $4.38(\mathrm{dd}, 1 \mathrm{H}, J=10.5,4.9 \mathrm{~Hz}$, 
H-6), 3.79 (t, 1H, $J=10.2 \mathrm{~Hz}, \mathrm{H}-6), 3.71(\mathrm{t}, 1 \mathrm{H}, J=9.5 \mathrm{~Hz}, \mathrm{H}-4)$, 3.62-3.53 (m, $2 \mathrm{H}, \mathrm{H}-2, \mathrm{H}-5) .{ }^{13} \mathrm{C}-\mathrm{APT}$ NMR $\left(\mathrm{CDCl}_{3}, 101 \mathrm{MHz}\right.$, HSQC): $\delta 165.3(\mathrm{C}=\mathrm{O} \mathrm{Bz}), 136.7\left(\mathrm{C}_{\mathrm{q}}\right), 133.7,133.4\left(\mathrm{CH}_{\text {arom }}\right)$, $130.8\left(\mathrm{C}_{\mathrm{q}}\right), 129.9,129.3\left(\mathrm{CH}_{\text {arom }}\right), 129.2\left(\mathrm{C}_{\mathrm{q}}\right), 129.1,128.8,128.5$, 128.2, $126.1\left(\mathrm{CH}_{\text {arom }}\right), 101.3(\mathrm{CHPh}), 87.1(\mathrm{C}-1), 78.4(\mathrm{C}-4), 73.5$ (C-3), 70.7 (C-5), 68.3 (C-6), 63.9 (C-2). HRMS: $[\mathrm{M}+\mathrm{H}]^{+}$calcd for $\mathrm{C}_{26} \mathrm{H}_{24} \mathrm{~N}_{3} \mathrm{O}_{5} \mathrm{~S}$ 490.14312, found 490.14305 .

Phenyl 2-Amino-3-O-benzyl-4,6-O-benzylidene-2-deoxy-1-thio$\beta$-D-glucopyranoside (16). Fully protected glycoside $15^{35}$ (9.11 g, $15.7 \mathrm{mmol}$ ) was dissolved in $160 \mathrm{~mL}$ of EtOH and heated to reflux, after which ethylene diamine $(52 \mathrm{~mL}, 785 \mathrm{mmol}, 50$ equiv) was added in three portions and reflux was maintained overnight. The reaction mixture was concentrated under reduced pressure and mixed with toluene $(100 \mathrm{~mL})$ and $45 \mathrm{~g}$ of silica gel, and the mixture was evaporated to dryness. Column chromatography ( $8: 2$ to $2: 1$ pentane/ EtOAc) gave the free amine as a white solid $(6.19 \mathrm{~g}, 13.76 \mathrm{mmol}$, $88 \%)$ that could be recrystallized in EtOAc/petroleum ether. $R_{\mathrm{f}} 0.40$ (2:1 pentane/EtOAc). mp $136.1-137.5^{\circ} \mathrm{C} .[\alpha]_{\mathrm{D}}^{20}=-33.5^{\circ}(c=0.57$, $\mathrm{CHCl}_{3}$ ). IR (thin film): 698, 748, 1026, 1069, 1123, 1371, 1452, 1583, 2870, 3030, 3059. ${ }^{1} \mathrm{H}$ NMR ( $\mathrm{CDCl}_{3}, 400 \mathrm{MHz}, \mathrm{H}-\mathrm{H}$ COSY, HSQC): $\delta 7.56-7.44\left(\mathrm{~m}, 4 \mathrm{H}, \mathrm{CH}_{\text {arom }}\right), 7.42-7.24\left(\mathrm{~m}, 11 \mathrm{H}, \mathrm{CH}_{\text {arom }}\right), 5.59(\mathrm{~s}$, $1 \mathrm{H}, \mathrm{CHPh}), 4.99$ (d, $1 \mathrm{H}, J=11.3 \mathrm{~Hz}, \mathrm{CHH} \mathrm{Bn}), 4.68$ (d, $1 \mathrm{H}, J=11.2$ $\mathrm{Hz}, \mathrm{CHH} \mathrm{Bn}$ ), 4.58 (d, $1 \mathrm{H}, J=9.9 \mathrm{~Hz}, \mathrm{H}-1), 4.38$ (dd, $1 \mathrm{H}, J=10.5$, $5.0 \mathrm{~Hz}, \mathrm{H}-6), 3.81(\mathrm{t}, 1 \mathrm{H}, J=10.3 \mathrm{~Hz}, \mathrm{H}-6), 3.72(\mathrm{t}, 1 \mathrm{H}, J=9.2 \mathrm{~Hz}$, $\mathrm{H}-4), 3.59$ (t, $1 \mathrm{H}, J=9.0 \mathrm{~Hz}, \mathrm{H}-3$ ), 3.52 (td, $1 \mathrm{H}, J=9.7,4.9 \mathrm{~Hz}, \mathrm{H}-5)$, $2.91(\mathrm{t}, 1 \mathrm{H}, J=9.4 \mathrm{~Hz}, \mathrm{H}-2), 1.75\left(\mathrm{bs}, 2 \mathrm{H}, \mathrm{NH}_{2}\right) .{ }^{13} \mathrm{C}-\mathrm{APT}$ NMR $\left(\mathrm{CDCl}_{3}, 101 \mathrm{MHz}, \mathrm{HSQC}\right): \delta$ 138.2, $137.4\left(\mathrm{C}_{\mathrm{q}}\right), 133.0\left(\mathrm{CH}_{\text {arom }}\right)$, $131.8\left(\mathrm{C}_{\mathrm{q}} \mathrm{SPh}\right), 129.1,128.6,128.4,128.3,128.3,128.0,126.0$ $\left(\mathrm{CH}_{\text {arom }}\right), 101.3(\mathrm{CHPh}), 89.6(\mathrm{C}-1), 82.2,82.2(\mathrm{C} 3, \mathrm{C}-4), 75.1\left(\mathrm{CH}_{2}\right.$ $\mathrm{Bn}), 70.7$ (C-5), 68.8 (C-6), 55.5 (C-2). HRMS: $[\mathrm{M}+\mathrm{H}]^{+}$calcd for $\mathrm{C}_{26} \mathrm{H}_{28} \mathrm{NO}_{4} \mathrm{~S} 450.17336$, found 450.17238 .

1-(4-Nitrophenyl)-4-pyridone (17). Following the procedure of You and Twieg, ${ }^{64}$ 4-hydroxypyridine (14.3 g, $\left.150 \mathrm{mmol}\right)$, 4chloronitrobenzene $(22.9 \mathrm{~g}, 145 \mathrm{mmol})$, and $\mathrm{K}_{2} \mathrm{CO}_{3}(20.7 \mathrm{~g}, 150$ $\mathrm{mmol}$ ) were suspended in $\mathrm{N}$-methyl-2-pyrrolidone $(110 \mathrm{~mL})$ and heated at $150{ }^{\circ} \mathrm{C}$ for $2 \mathrm{~h}$. The hot solution was then poured directly onto ice and allowed to precipitate until all of the ice had melted. The suspension was then filtered and washed four times with cold $\mathrm{H}_{2} \mathrm{O}$. The resulting solid was dried under a vacuum at $100{ }^{\circ} \mathrm{C}$ until dry. Yield: $26.6 \mathrm{~g}, 123 \mathrm{mmol}, 85 \%$. IR (neat): 606, 692, 741, 752, 843, $1015,1111,1198,1285,1339,1495,1514,1582,1638,3071 .{ }^{1} \mathrm{H}$ NMR (DMSO, $400 \mathrm{MHz}, \mathrm{H}-\mathrm{H}$ COSY, HSQC $): \delta 8.38(\mathrm{~d}, 2 \mathrm{H}, J=9.1 \mathrm{~Hz})$, $8.14(\mathrm{~d}, 2 \mathrm{H}, J=7.8 \mathrm{~Hz}), 7.86(\mathrm{~d}, 2 \mathrm{H}, J=9.1 \mathrm{~Hz}), 6.29(\mathrm{~d}, 2 \mathrm{H}, J=7.8$ $\mathrm{Hz}) .{ }^{13} \mathrm{C}-\mathrm{APT}$ NMR (DMSO, $101 \mathrm{MHz}$, HSQC): $\delta$ 177.6, 147.1, 145.9, 139.2, 125.3, 123.2, 118.3. HRMS: $[\mathrm{M}+\mathrm{H}]^{+}$calcd for $\mathrm{C}_{11} \mathrm{H}_{9} \mathrm{~N}_{2} \mathrm{O}_{3}$ 217.06077, found 217.06074.

3,5-Dinitro-1-(4-nitrophenyl)-4-pyridone (18). In a modification of the procedure of Matsumura et al., ${ }^{30}$ an ice-cooled three-neck flask equipped with a condenser was charged with $120 \mathrm{~mL}$ of $\mathrm{H}_{2} \mathrm{SO}_{4}(30 \%$ $\mathrm{SO}_{3}$ ), and then $120 \mathrm{~mL}$ of fuming $99 \% \mathrm{HNO}_{3}$ was slowly added. To this cold mixture was added pyridone $17(21.6 \mathrm{~g}, 100 \mathrm{mmol})$ in small portions. When addition was complete, the mixture was slowly brought to $130{ }^{\circ} \mathrm{C}$ and stirred for $40 \mathrm{~h}$. The mixture was allowed to cool and then poured over ice, stirred for $3 \mathrm{~h}$, filtered, and washed three times with cold water. Yield: $18.4 \mathrm{~g}, 60 \mathrm{mmol}, 60 \%$. Purity (NMR): 90\%. Tetranitro [3,5-dinitro-1-(2,4-dinitrophenyl)-4-pyridone ${ }^{1} \mathrm{H}$ NMR (DMSO, $400 \mathrm{MHz}$ ): $\delta 9.42(\mathrm{~s}, 2 \mathrm{H}), 9.05(\mathrm{~d}, 1 \mathrm{H}, J$ $=2.6 \mathrm{~Hz}), 8.87(\mathrm{dd}, 1 \mathrm{H}, J=8.8,2.6 \mathrm{~Hz}), 8.32(\mathrm{~d}, 1 \mathrm{H}, J=8.7 \mathrm{~Hz})]$ and dinitro [3-nitro-1-(4-nitrophenyl)-4-pyridone ${ }^{1} \mathrm{H}$ NMR (DMSO, 400 $\mathrm{MHz}): \delta 9.18(\mathrm{~d}, 1 \mathrm{H}, J=2.5 \mathrm{~Hz}), 8.43(\mathrm{~d}, 2 \mathrm{H}, J=9.0 \mathrm{~Hz}), 8.26(\mathrm{dd}$, $1 \mathrm{H}, J=7.8,2.5 \mathrm{~Hz}), 7.99(\mathrm{~d}, 2 \mathrm{H}, J=9.1 \mathrm{~Hz}), 6.68(\mathrm{~d}, 1 \mathrm{H}, J=7.9 \mathrm{~Hz})]$ impurities were present (ratios varied slightly upon repetition). IR (neat): 717, 768, 789, 853, 910, 1141, 1261, 1306, 1350, 1449, 1514, 1591, 1672, 3076. ${ }^{1} \mathrm{H}$ NMR (DMSO, $\left.400 \mathrm{MHz}\right): \delta 9.38(\mathrm{~s}, 1 \mathrm{H}), 8.47$ $(\mathrm{d}, 1 \mathrm{H}, J=9.0 \mathrm{~Hz}), 8.05(\mathrm{~d}, 1 \mathrm{H}, J=9.1 \mathrm{~Hz}) .{ }^{13} \mathrm{C}-\mathrm{APT}$ NMR (DMSO, $101 \mathrm{MHz}): \delta 159.3,147.6,145.5,142.1,141.6,125.7,125.1$. HRMS: $[\mathrm{M}+\mathrm{H}]^{+}$calcd for $\mathrm{C}_{11} \mathrm{H}_{7} \mathrm{~N}_{4} \mathrm{O}_{7} 307.03093$, found 307.03123.

Phenyl 2-(3,5-Dinitro-4-pyridone)-3-O-benzyl-4,6-O-benzylidene2-deoxy-1-thio- $\beta$-D-glucopyranoside (5). Free amine 16 (3.6 g, 8 $\mathrm{mmol})$ and reagent $18(2.7 \mathrm{~g}, 8.8 \mathrm{mmol}, 1.1$ equiv $)$ were dissolved in pyridine $(48 \mathrm{~mL})$ and $\mathrm{AcOH}(4 \mathrm{~mL})$ and left to stir for $30 \mathrm{~min}$. The mixture was diluted with EtOAc and washed with $1 \mathrm{M}$ aqueous $\mathrm{HCl}$ $(5 \times)$ and saturated aqueous $\mathrm{NaHCO}_{3}(1 \times)$. The organic layer was dried $\left(\mathrm{MgSO}_{4}\right)$, filtered, and concentrated under reduced pressure. Column chromatography: DCM until all of the nitroanaline had been removed, followed by $1 \%$ to $5 \%$ acetone in DCM. Yield: $4.84 \mathrm{~g}, 7.8$ mmol $(98 \%)$ as a yellow solid. $R_{\mathrm{f}} 0.21(\mathrm{DCM}),[\alpha]_{\mathrm{D}}^{20}=10.5^{\circ}(c=0.5$, $\mathrm{CHCl}_{3}$ ). IR (thin film): 604, 696, 746, 989, 1055, 1094, 1211, 1300, $1329,1516,1674,2856,2926,3034,3059 .{ }^{1} \mathrm{H}$ NMR (acetone- $d_{6}, 400$ $\mathrm{MHz}, \mathrm{H}-\mathrm{H}$ COSY, HSQC): $\delta 8.74$ (s, $2 \mathrm{H}, \mathrm{CH}$ pyridone), 7.63-7.54 $\left(\mathrm{m}, 2 \mathrm{H}, \mathrm{CH}_{\text {arom }}\right), 7.51-7.39\left(\mathrm{~m}, 5 \mathrm{H}, \mathrm{CH}_{\text {arom }}\right), 7.39-7.31(\mathrm{~m}, 3 \mathrm{H}$, $\left.\mathrm{CH}_{\text {arom }}\right), 7.21-7.14\left(\mathrm{~m}, 3 \mathrm{H}, \mathrm{CH}_{\text {arom }}\right), 7.14-7.07\left(\mathrm{~m}, 2 \mathrm{H}, \mathrm{CH}_{\text {arom }}\right)$, $5.84(\mathrm{~s}, 1 \mathrm{H}, \mathrm{CHPh}), 5.73(\mathrm{~d}, 1 \mathrm{H}, J=10.4 \mathrm{~Hz}, \mathrm{H}-1), 4.84(\mathrm{~d}, 1 \mathrm{H}, J=$ $12.1 \mathrm{~Hz}, \mathrm{CHH} \mathrm{Bn}), 4.62$ (d, $1 \mathrm{H}, J=12.1 \mathrm{~Hz}, \mathrm{CHH} \mathrm{Bn}$ ), 4.55-4.47 (m, 1H, H-3), 4.44-4.39 (m, 1H, H-6), 4.39 (t, 1H, J = 8.9 Hz, H-2), 4.06-3.91 (m, 3H, H-4, H-5, H-6). ${ }^{13}$ C-APT NMR (acetone- $d_{6}, 101$ $\mathrm{MHz}, \mathrm{HSQC}): \delta 159.9$ (C=O pyridone), $143.1\left(\mathrm{C}_{\mathrm{q}} \mathrm{NO}_{2}\right), 138.5$, $137.8\left(\mathrm{C}_{\mathrm{q}}\right), 133.4\left(\mathrm{CH}_{\text {arom }}\right), 131.7\left(\mathrm{C}_{\mathrm{q}} \mathrm{SPh}\right), 130.3,129.7,129.5$, 129.2, 129.0, 129.0, 127.0 ( $\left.\mathrm{CH}_{\text {arom }}\right), 102.0(\mathrm{CHPh}), 85.9(\mathrm{C}-1), 83.0$ (C-4), 77.0 (C-3), $74.7\left(\mathrm{CH}_{2} \mathrm{Bn}\right), 71.6$ (C-2), 70.9 (C-5), 68.8 (C-6). HRMS: $[\mathrm{M}+\mathrm{H}]^{+}$calcd for $\mathrm{C}_{31} \mathrm{H}_{28} \mathrm{~N}_{3} \mathrm{O}_{9} \mathrm{~S}$ 618.15408, found 618.15375 .

Trifluoromethanesulfonyl 2,3-Di-O-benzyl-4,6-O-benzylidene- $\alpha$ D-glucopyranoside (19). ${ }^{9}{ }^{1} \mathrm{H}$ NMR $\left(\mathrm{CD}_{2} \mathrm{Cl}_{2}, \mathrm{~T}=213 \mathrm{~K}, 400 \mathrm{MHz}\right.$, $\mathrm{H}-\mathrm{H}$ COSY, HSQC): $\delta 6.08$ (d, $1 \mathrm{H}, J=3.5 \mathrm{~Hz}, \mathrm{H}-1), 5.59(\mathrm{~s}, 1 \mathrm{H}$, $\mathrm{CHPh}), 4.89(\mathrm{~d}, 1 \mathrm{H}, J=11.0 \mathrm{~Hz}, \mathrm{CHH} \mathrm{Bn}), 4.85-4.69(\mathrm{~m}, 3 \mathrm{H}, \mathrm{CHH}$ $\mathrm{Bn}, \mathrm{CH}_{2} \mathrm{Bn}$ ), 4.29 (dd, $\left.1 \mathrm{H}, J=10.3,4.8 \mathrm{~Hz}, \mathrm{H}-6\right), 4.09-3.94(\mathrm{~m}, 2 \mathrm{H}$, $\mathrm{H}-3, \mathrm{H}-5), 3.86-3.70$ (m, 3H, H-2, H-4, H-6). ${ }^{13} \mathrm{C}-\mathrm{APT}$ NMR $\left(\mathrm{CD}_{2} \mathrm{Cl}_{2}, T=213 \mathrm{~K}, 101 \mathrm{MHz}, \mathrm{HSQC}\right): \delta 106.1(\mathrm{C}-1), 100.8$ (CHPh), 79.6 (C-4), 77.0 (C-3), 76.3 (C-2), 75.0, 74.1 ( $\left.\mathrm{CH}_{2} \mathrm{Bn}\right)$, 67.4 (C-6), 65.8 (C-5).

Trifluoromethanesulfonyl 2-Azido-3-O-benzyl-4,6-O-benzylidene-2-deoxy- $\alpha$-D-glucopyranoside (20). ${ }^{1} \mathrm{H}$ NMR $\left(\mathrm{CD}_{2} \mathrm{Cl}_{2}, T=\right.$ $243 \mathrm{~K}, 400 \mathrm{MHz}, \mathrm{H}-\mathrm{H}$ COSY, HSQC): $\delta 6.08(\mathrm{~d}, 1 \mathrm{H}, J=3.5 \mathrm{~Hz}, \mathrm{H}-$ 1), $5.64(\mathrm{~s}, 1 \mathrm{H}, \mathrm{CHPh}), 4.98(\mathrm{~d}, 1 \mathrm{H}, J=10.6 \mathrm{~Hz}, \mathrm{CHH} \mathrm{Bn}), 4.78$ (d, $1 \mathrm{H}, J=10.6 \mathrm{~Hz}, \mathrm{CHH} \mathrm{Bn}$ ), 4.32 (dd, $1 \mathrm{H}, J=10.4,4.9 \mathrm{~Hz}, \mathrm{H}-6)$, 4.11-4.00 (m, 2H, H-3, H-5), 3.94-3.86 (m, 2H, H-2, H-4), $3.82(\mathrm{t}$, $1 \mathrm{H}, J=10.3 \mathrm{~Hz}, \mathrm{H}-6) .{ }^{13} \mathrm{C}-\mathrm{APT}$ NMR $\left(\mathrm{CD}_{2} \mathrm{Cl}_{2}, T=243 \mathrm{~K}, 101 \mathrm{MHz}\right.$, HSQC): $\delta 137.2,136.7\left(\mathrm{C}_{\mathrm{q}}\right), 130.5,128.4,128.4,125.9\left(\mathrm{CH}_{\text {arom }}\right)$, 105.0 (C-1), 101.3 (CHPh), 80.6 (C-4), 76.4 (C-3), $75.3\left(\mathrm{CH}_{2} \mathrm{Bn}\right)$, 67.6 (C-6), 66.2 (C-5), 61.4 (C-2).

Trifluoromethanesulfonyl 2-Azido-3-O-benzoyl-4,6-O-benzylidene-2-deoxy- $\alpha$-D-glucopyranoside (21). ${ }^{1} \mathrm{H}$ NMR $\left(\mathrm{CD}_{2} \mathrm{Cl}_{2}, T=\right.$ $243 \mathrm{~K}, 400 \mathrm{MHz}, \mathrm{H}-\mathrm{H}$ COSY, HSQC): $\delta 6.23(\mathrm{~d}, 1 \mathrm{H}, J=3.5 \mathrm{~Hz}, \mathrm{H}-$ 1), $5.80(\mathrm{t}, 1 \mathrm{H}, J=10.0 \mathrm{~Hz}, \mathrm{H}-3), 5.54(\mathrm{~s}, 1 \mathrm{H}, \mathrm{CHPh}), 4.36(\mathrm{dd}, 1 \mathrm{H}, J$ $=10.4,4.9 \mathrm{~Hz}, \mathrm{H}-6), 4.21(\mathrm{td}, 1 \mathrm{H}, J=9.9,4.9 \mathrm{~Hz}, \mathrm{H}-5), 4.12(\mathrm{dd}, 1 \mathrm{H}$, $J=10.2,3.5 \mathrm{~Hz}, \mathrm{H}-2), 3.98(\mathrm{t}, 1 \mathrm{H}, J=9.8 \mathrm{~Hz}, \mathrm{H}-4), 3.86(\mathrm{t}, 1 \mathrm{H}, J=$ $10.3 \mathrm{~Hz}, \mathrm{H}-6) .{ }^{13} \mathrm{C}-\mathrm{APT}$ NMR $\left(\mathrm{CD}_{2} \mathrm{Cl}_{2}, \mathrm{~T}=243 \mathrm{~K}, 101 \mathrm{MHz}\right.$, HSQC): $\delta 104.5$ (C-1), 101.8 (CHPh), 77.5 (C-4), 69.3 (C-3), 67.6 (C-6), 66.4 (C-5), 60.9 (C-2).

Trifluoromethanesulfonyl 2-Azido-3-O-benzyl-2-deoxy-4,6-O-ditert-butylsilylidene- $\alpha$-D-glucopyranoside (22). ${ }^{1} \mathrm{H}$ NMR $\left(\mathrm{CD}_{2} \mathrm{Cl}_{2}, \mathrm{~T}\right.$ $=233 \mathrm{~K}, 400 \mathrm{MHz}, \mathrm{H}-\mathrm{H}$ COSY, HSQC, HMBC): $\delta 6.00$ (d, 1H, $J=$ $3.4 \mathrm{~Hz}, \mathrm{H}-1), 5.08$ (d, $1 \mathrm{H}, J=10.1 \mathrm{~Hz}, \mathrm{CHH} \mathrm{Bn}), 4.81(\mathrm{~d}, 1 \mathrm{H}, J=$ $10.2 \mathrm{~Hz}, \mathrm{CHH} \mathrm{Bn}$ ), 4.15-4.06 (m, 2H, H-4, H-6), 3.95-3.84 (m, 3H, $\mathrm{H}-3, \mathrm{H}-5, \mathrm{H}-6), 3.79$ (dd, $1 \mathrm{H}, J=10.1,3.4 \mathrm{~Hz}, \mathrm{H}-2), 1.07$ (s, 9H, $\mathrm{CH}_{3}$ $\left.{ }^{\mathrm{t}} \mathrm{Bu}\right), 1.00\left(\mathrm{~s}, 9 \mathrm{H}, \mathrm{CH}_{3}{ }^{\mathrm{t}} \mathrm{Bu}\right) .{ }^{13} \mathrm{C}-\mathrm{APT}$ NMR $\left(\mathrm{CD}_{2} \mathrm{Cl}_{2}, \mathrm{~T}=233 \mathrm{~K}, 101\right.$ $\mathrm{MHz}$, HSQC, HMBC): $\delta 118.9$ (q, $\left.J=317.6 \mathrm{~Hz}, \mathrm{CF}_{3}\right), 104.8(\mathrm{C}-1)$, 78.8 (C-3), 76.9 (C-4), $75.7\left(\mathrm{CH}_{2} \mathrm{Bn}\right), 70.0$ (C-5), 65.3 (C-6), 60.6 (C-2), 27.0, $26.4\left(\mathrm{CH}_{3}{ }^{\mathrm{t}} \mathrm{Bu}\right), 22.5,19.7\left(\mathrm{C}_{\mathrm{q}}{ }^{\mathrm{t}} \mathrm{Bu}\right) .{ }^{13} \mathrm{C}-\mathrm{HMBC}$ NMR $\left(\mathrm{CD}_{2} \mathrm{Cl}_{2}, 101 \mathrm{MHz}\right): \delta 104.8\left(J_{\mathrm{C} 1-\mathrm{H} 1}=187 \mathrm{~Hz}, \mathrm{C}-1\right)$.

3-O-Benzyl-4,6-O-benzylidene-2-deoxy-2-(3,5-dinitro-4-pyridone)-D-glucal (24). Off-white solid. $R_{\mathrm{f}} 0.20$ (7:3 pentane/EtOAc). $[\alpha]_{\mathrm{D}}^{23}=+85.9^{\circ}(c=0.32, \mathrm{DCM})$. IR (thin film): 698, 720, 753, 1007, $1059,1095,1192,1247,1304,1351,1516,1679,2880,2924,3072 .{ }^{1} \mathrm{H}$ NMR (acetone- $d_{6}, 500 \mathrm{MHz}, \mathrm{H}-\mathrm{H}$ COSY, HSQC) $\delta 8.72(\mathrm{~s}, 2 \mathrm{H}$, $\mathrm{CH}$ pyridone), $7.62-7.53\left(\mathrm{~m}, 2 \mathrm{H}, \mathrm{CH}_{\text {arom }}\right), 7.49-7.37(\mathrm{~m}, 4 \mathrm{H}$, $\left.\mathrm{CH}_{\text {arom }}, \mathrm{H}-1\right), 7.29-7.14\left(\mathrm{~m}, 5 \mathrm{H}, \mathrm{CH}_{\text {arom }}\right), 5.88(\mathrm{~s}, 1 \mathrm{H}, \mathrm{CHPh}), 4.93-$ $4.88(\mathrm{~m}, 2 \mathrm{H}, \mathrm{CHH} \mathrm{Bn}, \mathrm{H}-3), 4.68(\mathrm{~d}, 1 \mathrm{H}, J=11.8 \mathrm{~Hz}, \mathrm{CHH} \mathrm{Bn}), 4.46$ (dd, $1 \mathrm{H}, J=10.5,5.2 \mathrm{~Hz}, \mathrm{H}-6), 4.37(\mathrm{dd}, 1 \mathrm{H}, J=10.4,6.9 \mathrm{~Hz}, \mathrm{H}-4)$, 
$4.30(\mathrm{td}, 1 \mathrm{H}, J=10.2,5.1 \mathrm{~Hz}, \mathrm{H}-5), 4.03(\mathrm{t}, 1 \mathrm{H}, J=10.3 \mathrm{~Hz}, \mathrm{H}-6)$. ${ }^{13} \mathrm{C}$-APT NMR (acetone- $\left.d_{6}, 101 \mathrm{MHz}, \mathrm{HSQC}\right): \delta 160.0(\mathrm{C}=\mathrm{O}$ pyridone), 149.3 (C-1), 144.7 ( $\mathrm{CH}$ pyridone), $142.8\left(\mathrm{C}_{\mathrm{q}} \mathrm{NO}_{2}\right), 138.4$ $\left(\mathrm{C}_{\mathrm{q}} \mathrm{Bn}, \mathrm{Ph}\right), 129.8,129.2,129.1,129.0,128.8,127.0\left(\mathrm{CH}_{\text {arom }}\right), 122.1$ (C-2), 101.9 (CHPh), 80.2 (C-4), $74.7\left(\mathrm{CH}_{2} \mathrm{Bn}\right), 74.6(\mathrm{C}-3), 70.7$ (C-5), 68.2 (C-6). HRMS: $[\mathrm{M}+\mathrm{H}]^{+}$calcd for $\mathrm{C}_{25} \mathrm{H}_{22} \mathrm{~N}_{3} \mathrm{O}_{9}$ 508.13506, found 508.13465.

Ethyl 2-Azido-3-O-benzyl-2-deoxy-4,6-O-di-tert-butylsilylidene- $\beta$ $D$-glucopyranoside (2A). Donor 2 and ethanol were condensed using the general procedure for $\mathrm{Tf}_{2} \mathrm{O} / \mathrm{Ph}_{2} \mathrm{SO}$-mediated glycosylations and purified by flash column chromatography ( $0 \%$ to $5 \% \mathrm{Et}_{2} \mathrm{O}$ in pentane) to yield glycosylation product $2 \mathrm{~A}(30 \mathrm{mg}, 65 \mu \mathrm{mol}, 65 \%, \alpha / \beta<1: 20)$ as a colorless oil. $R_{\mathrm{f}} 0.35\left(5 \% \mathrm{Et}_{2} \mathrm{O}\right.$ in pentane). $[\alpha]_{\mathrm{D}}^{23}=-69.6^{\circ}(c=$ $0.5, \mathrm{CHCl}_{3}$ ). IR (neat): 652, 768, 827, 962, 1082, 1161, 1474, 2112, 2859, 2932. ${ }^{1} \mathrm{H}$ NMR ( $\mathrm{CDCl}_{3}, 400 \mathrm{MHz}, \mathrm{H}-\mathrm{H}$ COSY, HSQC): $\delta$ 7.46-7.40 (m, 2H, $\left.\mathrm{CH}_{\text {arom }}\right), 7.39-7.27\left(\mathrm{~m}, 3 \mathrm{H}, \mathrm{CH}_{\text {arom }}\right), 4.99(\mathrm{~d}, 1 \mathrm{H}$, $J=11.0 \mathrm{~Hz}, \mathrm{CHH} \mathrm{Bn}), 4.81(\mathrm{~d}, 1 \mathrm{H}, J=10.9 \mathrm{~Hz}, \mathrm{CHH} \mathrm{Bn}), 4.31$ (dd, $1 \mathrm{H}, J=7.7,1.7 \mathrm{~Hz}, \mathrm{H}-1), 4.16$ (dd, $1 \mathrm{H}, J=10.3,5.0 \mathrm{~Hz}, \mathrm{H}-6), 3.98-$ $3.87\left(\mathrm{~m}, 3 \mathrm{H}, \mathrm{CHH}-\mathrm{CH}_{3} \mathrm{Et}, \mathrm{H}-4, \mathrm{H}-6\right), 3.61(\mathrm{dq}, 1 \mathrm{H}, J=9.5,7.1 \mathrm{~Hz}$, $\mathrm{CHH}-\mathrm{CH}_{3} \mathrm{Et}$ ), 3.41-3.28 (m, 3H, H-2, H-3, H-5), 1.26 (t, 3H, J = $\left.7.1 \mathrm{~Hz}, \mathrm{CH}_{3} \mathrm{Et}\right), 1.08$ (s, 9H, $\left.\mathrm{CH}_{3}{ }^{t} \mathrm{Bu}\right), 1.01\left(\mathrm{~s}, 9 \mathrm{H}, \mathrm{CH}_{3}{ }^{\mathrm{t}} \mathrm{Bu}\right) .{ }^{13} \mathrm{C}-$ APT NMR ( $\left.\mathrm{CDCl}_{3}, 101 \mathrm{MHz}, \mathrm{HSQC}\right): \delta 138.3\left(\mathrm{C}_{\mathrm{q}}\right), 128.5,128.4$, $128.0\left(\mathrm{CH}_{\text {arom }}\right), 102.1(\mathrm{C}-1), 82.4(\mathrm{C}-3), 78.1(\mathrm{C}-4), 75.4\left(\mathrm{CH}_{2} \mathrm{Bn}\right)$, 70.5 (C-5), $66.4(\mathrm{C}-6), 66.1\left(\mathrm{CH}_{2} \mathrm{Et}\right), 65.6(\mathrm{C}-2), 27.6,27.2\left(\mathrm{CH}_{3}\right.$ $\left.{ }^{\mathrm{t}} \mathrm{Bu}\right), 22.8,20.1\left(\mathrm{C}_{\mathrm{q}}{ }^{\mathrm{t}} \mathrm{Bu}\right), 15.2\left(\mathrm{CH}_{3} \mathrm{Et}\right)$. HRMS: $\left[\mathrm{M}-\mathrm{N}_{2}+\mathrm{H}\right]^{+}$ calcd for $\mathrm{C}_{23} \mathrm{H}_{38} \mathrm{NO}_{5} \mathrm{Si} 436.25138$, found 436.25132 .

Cyclohexyl 2-Azido-3-O-benzyl-2-deoxy-4,6-O-di-tert-butylsilylidene- $\beta$-D-glucopyranoside (2B). Donor 2 and cyclohexanol were condensed using the general procedure for $\mathrm{Tf}_{2} \mathrm{O} / \mathrm{Ph}_{2} \mathrm{SO}$-mediated glycosylations and purified by flash column chromatography (4:1 to $0: 1$ pentane/toluene) to yield glycosylation product $2 \mathrm{~B}(40 \mathrm{mg}, 77$ $\mu \mathrm{mol}, 77 \%, \alpha / \beta<1: 20)$ as a colorless oil. $R_{\mathrm{f}} 0.43\left(5 \% \mathrm{Et}_{2} \mathrm{O}\right.$ in pentane). $[\alpha]_{\mathrm{D}}^{20}=-44.3^{\circ}\left(c=1.0, \mathrm{CHCl}_{3}\right)$. IR (thin film): 696, 768, 827, 961, 1080, 1163, 1364, 2112, 2859, 2934. ${ }^{1} \mathrm{H} \mathrm{NMR}\left(\mathrm{CDCl}_{3}, 400\right.$ MHz, H-H COSY, HSQC): $\delta 7.45-7.40\left(\mathrm{~m}, 2 \mathrm{H}, \mathrm{CH}_{\text {arom }}\right), 7.38-7.27$ (m, 3H, $\mathrm{CH}_{\text {arom }}$ ), 4.97 (d, $\left.1 \mathrm{H}, J=11.1 \mathrm{~Hz}, \mathrm{CHH} \mathrm{Bn}\right), 4.81(\mathrm{~d}, 1 \mathrm{H}, J=$ $11.1 \mathrm{~Hz}, \mathrm{CHH} \mathrm{Bn}$ ), 4.42 (d, $1 \mathrm{H}, J=7.8 \mathrm{~Hz}, \mathrm{H}-1), 4.15$ (dd, $1 \mathrm{H}, J=$ 10.3, 5.0 Hz, H-6), 3.99-3.89 (m, $2 \mathrm{H}, \mathrm{H}-3, \mathrm{H}-6), 3.64(\mathrm{tt}, 2 \mathrm{H}, J=9.2$, $3.8 \mathrm{~Hz}, \mathrm{CH} \mathrm{Cy}), 3.40-3.24$ (m, 3H, H-2, H-4, H-5), 1.96-1.83 (m, $\left.2 \mathrm{H}, \mathrm{CH}_{2} \mathrm{Cy}\right), 1.80-1.71\left(\mathrm{~m}, 2 \mathrm{H}, \mathrm{CH}_{2} \mathrm{Cy}\right), 1.55-1.48\left(\mathrm{~m}, 1 \mathrm{H}, \mathrm{CH}_{2}\right.$ $\mathrm{Cy}), 1.47-1.37$ (m, 2H, $\left.\mathrm{CH}_{2} \mathrm{Cy}\right), 1.34-1.20$ (m, 3H, $\left.\mathrm{CH}_{2} \mathrm{Cy}\right), 1.08$ $\left(\mathrm{s}, 9 \mathrm{H},{ }^{\mathrm{t}} \mathrm{Bu}\right), 1.01\left(\mathrm{~s}, 9 \mathrm{H},{ }^{\mathrm{t}} \mathrm{Bu}\right) .{ }^{13} \mathrm{C}-\mathrm{APT}$ NMR $\left(\mathrm{CDCl}_{3}, 101 \mathrm{MHz}\right.$, HSQC): $\delta 138.4\left(\mathrm{C}_{\mathrm{q}}\right), 128.5,128.3,127.9\left(\mathrm{CH}_{\text {arom }}\right), 100.7(\mathrm{C}-1), 82.3$ (C-4), $78.3(\mathrm{CH} \mathrm{Cy}), 78.0(\mathrm{C}-3), 75.4\left(\mathrm{CH}_{2} \mathrm{Bn}\right), 70.5$ (C-5), 66.4 (C6), 65.8 (C-2), 33.6, $31.7\left(\mathrm{CH}_{2} \mathrm{Cy}\right), 27.6,27.2\left(\mathrm{CH}_{3}{ }^{\mathrm{t}} \mathrm{Bu}\right), 25.6\left(\mathrm{CH}_{2}\right.$ Cy), 24.1, $23.9\left(\mathrm{Cq}{ }^{\mathrm{t}} \mathrm{Bu}\right), 22.8,20.1\left(\mathrm{CH}_{2} \mathrm{Cy}\right)$. HRMS: $\left[\mathrm{M}-\mathrm{N}_{2}+\right.$ $\mathrm{H}]^{+}$calcd for $\mathrm{C}_{27} \mathrm{H}_{44} \mathrm{NO}_{5} \mathrm{Si} 490.29833$, found 490.29811.

Methyl 6-O-(2-Azido-3-O-benzyl-2-deoxy-4,6-O-di-tert-butylsilylidene- $\alpha / \beta$-D-glucopyranosyl)-2,3,4-tri-O-benzyl- $\alpha$-D-glucopyranoside (2C). Donor 2 and acceptor 25 were condensed using the general procedure for $\mathrm{Tf}_{2} \mathrm{O} / \mathrm{Ph}_{2} \mathrm{SO}$-mediated glycosylations (for an additional $18 \mathrm{~h}$ at $-40^{\circ} \mathrm{C}$ ) and purified by flash column chromatography (1:0 to 9:1 pentane/EtOAc) to yield glycosylation product $2 \mathrm{C}(81 \mathrm{mg}, 92$ $\mu \mathrm{mol}, 92 \%, \alpha / \beta=1: 14$ ) as a white solid. $R_{\mathrm{f}} 0.42$ (4:1 pentane/ EtOAc). $[\alpha]_{\mathrm{D}}^{23}=-18.6^{\circ}\left(c=1.0, \mathrm{CHCl}_{3}\right)$. IR (thin film): 654, 969, $735,827,962,1028,1070,1161,1362,1454,2112,2859,2931 .{ }^{1} \mathrm{H}$ $\mathrm{NMR}\left(\mathrm{CDCl}_{3}, 400 \mathrm{MHz}, \mathrm{H}-\mathrm{H}\right.$ COSY, HSQC, HMBC): $\delta 7.45-7.39$ $\left(\mathrm{m}, 2 \mathrm{H}, \mathrm{CH}_{\text {arom }}\right), 7.38-7.25\left(\mathrm{~m}, 18 \mathrm{H}, \mathrm{CH}_{\text {arom }}\right), 4.99(\mathrm{~d}, 1 \mathrm{H}, J=11.0$ $\mathrm{Hz}, \mathrm{CHH} \mathrm{Bn}), 4.98(\mathrm{~d}, 1 \mathrm{H}, J=10.9 \mathrm{~Hz}, \mathrm{CHH} \mathrm{Bn}), 4.94(\mathrm{~d}, 1 \mathrm{H}, J=$ $11.1 \mathrm{~Hz}, \mathrm{CHH} \mathrm{Bn}), 4.85-4.76$ (m, 3H, CHH Bn, $2 \times \mathrm{CHH} \mathrm{Bn}), 4.66$ $(\mathrm{d}, 1 \mathrm{H}, J=11.1 \mathrm{~Hz}, \mathrm{CHH} \mathrm{Bn}), 4.64(\mathrm{~d}, 1 \mathrm{H}, J=12.1 \mathrm{~Hz}, \mathrm{CHH} \mathrm{Bn})$, $4.60(\mathrm{~d}, 1 \mathrm{H}, J=3.6 \mathrm{~Hz}, \mathrm{H}-1), 4.17\left(\mathrm{~d}, 1 \mathrm{H}, J=7.9 \mathrm{~Hz}, \mathrm{H}-1^{\prime}\right), 4.15-$ 4.10 (m, 1H, H-6'), 4.05-3.96 (m, 2H, H-3, H-6), 3.96-3.87 (m, 2H, $\left.\mathrm{H}-4^{\prime}, \mathrm{H}-6^{\prime}\right), 3.76$ (ddd, $\left.1 \mathrm{H}, J=9.9,4.2,1.7 \mathrm{~Hz}, \mathrm{H}-5\right), 3.70(\mathrm{dd}, 1 \mathrm{H}, J=$ 10.7, 4.2 Hz, H-6), 3.59 (t, 1H, J = 9.5 Hz, H-4), 3.54 (dd, $1 \mathrm{H}, J=9.6$, $3.5 \mathrm{~Hz}, \mathrm{H}-2), 3.40\left(\mathrm{dd}, 1 \mathrm{H}, J=9.7,7.9 \mathrm{~Hz}, \mathrm{H}-2^{\prime}\right), 3.37-3.26(\mathrm{~m}, 5 \mathrm{H}$, $\mathrm{CH}_{3}$ Ome, $\left.\mathrm{H}-3^{\prime}, \mathrm{H}-5^{\prime}\right), 1.07$ (s, $\left.9 \mathrm{H}, \mathrm{CH}_{3}{ }^{\mathrm{t}} \mathrm{Bu}\right), 1.01$ (s, $\left.9 \mathrm{H}, \mathrm{CH}_{3}{ }^{\mathrm{t}} \mathrm{Bu}\right)$. ${ }^{13} \mathrm{C}$-APT NMR ( $\mathrm{CDCl}_{3}, 101 \mathrm{MHz}$, HSQC, HMBC): $\delta$ 138.9, 138.6, 138.2, 138.1 $\left(\mathrm{C}_{\mathrm{q}}\right)$, 128.6, 128.5, 128.5, 128.4, 128.3, 128.1, 128.0, 128.0, 127.9, 127.8, $127.7\left(\mathrm{CH}_{\text {arom }}\right), 102.2\left(\mathrm{C}-1^{\prime}\right), 98.3(\mathrm{C}-1), 82.5$ (C-
3'), 82.2 (C-3), 79.9 (C-2), 77.9 (C-4'), 77.7 (C-4), 75.9, 75.4, 75.0, $73.6\left(\mathrm{CH}_{2} \mathrm{Bn}\right), 70.6\left(\mathrm{C}-5^{\prime}\right), 69.7(\mathrm{C}-5), 68.6(\mathrm{C}-6), 66.3\left(\mathrm{C}-6^{\prime}\right), 65.6$ $\left(\mathrm{C}-2^{\prime}\right), 55.3(\mathrm{OMe}), 27.5,27.1\left(\mathrm{CH}_{3}{ }^{\mathrm{t}} \mathrm{Bu}\right), 22.8,20.1\left(\mathrm{C}_{\mathrm{q}}{ }^{\mathrm{t}} \mathrm{Bu}\right)$. Diagnostic peaks for the $\alpha$-anomer: ${ }^{1} \mathrm{H}$ NMR $\left(\mathrm{CDCl}_{3}, 400 \mathrm{MHz}\right): \delta$ $4.87\left(\mathrm{~d}, 1 \mathrm{H}, J=3.6 \mathrm{~Hz}, \mathrm{H}-\mathrm{1}^{\prime}\right), 4.52(\mathrm{~d}, 1 \mathrm{H}, J=3.4 \mathrm{~Hz}, \mathrm{H}-1) .{ }^{13} \mathrm{C}-\mathrm{APT}$ NMR $\left(\mathrm{CDCl}_{3}, 101 \mathrm{MHz}\right): \delta$ 98.1, 98.0, 68.3. HRMS: $\left[\mathrm{M}+\mathrm{NH}_{4}\right]^{+}$ calcd for $\mathrm{C}_{49} \mathrm{H}_{67} \mathrm{~N}_{4} \mathrm{O}_{10} \mathrm{Si} 899.46210$, found 899.46246.

2-Fluoroethyl 2-Azido-3-O-benzyl-2-deoxy-4,6-O-di-tert-butylsilylidene- $\alpha / \beta$-D-glucopyranoside (2D). Donor 2 and 2 -fluoroethanol were condensed using the general procedure for $\mathrm{Tf}_{2} \mathrm{O} / \mathrm{Ph}_{2} \mathrm{SO}$ mediated glycosylations and purified by flash column chromatography (1:0 to $0: 1$ pentane/toluene to $2 \% \mathrm{Et}_{2} \mathrm{O}$ in toluene) to yield glycosylation product $2 \mathrm{D}(37.8 \mathrm{mg}, 79 \mu \mathrm{mol}, 79 \%, \alpha / \beta=1: 5.5)$ as a colorless oil. $R_{\mathrm{f}} 0.20$ (toluene). Data reported for a 1.00:0.18 mixture of anomers: IR (neat): 654, 768, 827, 962, 1080, 1161, 1472, 2112, 2859, 2932. ${ }^{1} \mathrm{H}$ NMR $\left(\mathrm{CDCl}_{3}, 400 \mathrm{MHz}, \mathrm{H}-\mathrm{H}\right.$ COSY, HSQC): $\delta$ 7.45-7.40 (m, $\left.2.36 \mathrm{H}, \mathrm{CH}_{\text {arom }}\right), 7.39-7.27\left(\mathrm{~m}, 3.54 \mathrm{H}, \mathrm{CH}_{\text {arom }}\right), 5.06$ $\left(\mathrm{d}, 0.18 \mathrm{H}, J=10.7 \mathrm{~Hz}, \mathrm{CHH} \mathrm{Bn}{ }_{\alpha}\right), 4.99(\mathrm{~d}, 1 \mathrm{H}, J=10.9 \mathrm{~Hz}, \mathrm{CHH}$ $\left.\mathrm{Bn}_{\beta}\right), 4.86\left(\mathrm{~d}, 0.18 \mathrm{H}, J=3.6 \mathrm{~Hz}, \mathrm{H}-1_{\alpha}\right), 4.82(\mathrm{~d}, 0.18 \mathrm{H}, J=10.6 \mathrm{~Hz}$, $\left.\mathrm{CHH} \mathrm{Bn}_{\alpha}\right), 4.82\left(\mathrm{~d}, 1 \mathrm{H}, J=10.9 \mathrm{~Hz}, \mathrm{CHH} \mathrm{Bn}_{\beta}\right), 4.71-4.61(\mathrm{~m}$, $\left.1.18 \mathrm{H}, \mathrm{CHHF}_{\alpha}, \mathrm{CHHF}_{\beta}\right), 4.58-4.47\left(\mathrm{~m}, 1.18 \mathrm{H}, \mathrm{CHHF}_{\alpha}, \mathrm{CHHF}_{\beta}\right)$, $4.37\left(\mathrm{~d}, 1 \mathrm{H}, J=7.6 \mathrm{~Hz}, \mathrm{H}-1_{\beta}\right), 4.17\left(\mathrm{dd}, 1 \mathrm{H}, J=10.3,5.1 \mathrm{~Hz}, \mathrm{H}-6_{\beta}\right)$, 4.12-3.78 (m, 5.26H, CH $-\mathrm{CH}_{2} \mathrm{~F}_{\alpha}, \mathrm{CH}_{2}-\mathrm{CH}_{2} \mathrm{~F}_{\beta}, \mathrm{H}-3_{\alpha}, \mathrm{H}-4_{\alpha}, \mathrm{H}-4_{\beta}$, $\left.\mathrm{H}-5_{\alpha}, \mathrm{H}-6_{\alpha}, \mathrm{H}-6_{\alpha}, \mathrm{H}-6_{\beta}\right), 3.44-3.29\left(\mathrm{~m}, 3.18 \mathrm{H}, \mathrm{H}-2_{\alpha}, \mathrm{H}-2_{\beta}, \mathrm{H}-3_{\beta}, \mathrm{H}-\right.$ $\left.5_{\beta}\right), 1.09\left(\mathrm{~s}, 1.62 \mathrm{H}, \mathrm{CH}_{3}{ }^{\mathrm{t}} \mathrm{Bu}_{\alpha}\right), 1.08\left(\mathrm{~s}, 9 \mathrm{H}, \mathrm{CH}_{3}{ }^{\mathrm{t}} \mathrm{Bu}_{\beta}\right), 1.03(\mathrm{~s}, 1.62 \mathrm{H}$ $\left.\mathrm{CH}_{3}{ }^{\mathrm{t}} \mathrm{Bu}_{\alpha}\right), 1.01\left(\mathrm{~s}, 9 \mathrm{H}, \mathrm{CH}_{3}{ }^{\mathrm{t}} \mathrm{Bu}_{\beta}\right) .{ }^{13} \mathrm{C}$-APT NMR $\left(\mathrm{CDCl}_{3}, 101\right.$ MHz, HSQC $): \delta 138.3\left(\mathrm{C}_{\mathrm{q}, \alpha}\right), 138.2\left(\mathrm{C}_{\mathrm{q}, \beta}\right), 128.6\left(\mathrm{CH}_{\text {arom }} \mathrm{Bn}_{\alpha}\right), 128.5$ $\left(\mathrm{CH}_{\text {arom }} \mathrm{Bn}_{\beta}\right), 128.5\left(\mathrm{CH}_{\text {arom }} \mathrm{Bn}_{\alpha}\right), 128.4\left(\mathrm{CH}_{\text {arom }} \mathrm{Bn}_{\beta}\right), 128.0$ $\left(\mathrm{CH}_{\text {arom }} \mathrm{Bn}_{\alpha, \beta}\right), 102.4\left(\mathrm{C}-1_{\beta}\right), 98.3\left(\mathrm{C}-1_{\alpha}\right), 82.7(\mathrm{~d}, J=170.0 \mathrm{~Hz}$, $\left.\mathrm{CH}_{2} \mathrm{~F}_{\beta}\right), 82.4\left(\mathrm{~d}, J=170.6 \mathrm{~Hz}, \mathrm{CH}_{2} \mathrm{~F}_{\alpha}\right), 82.3\left(\mathrm{C}-3_{\beta}\right), 79.3,79.0\left(\mathrm{C}-3_{\alpha}\right.$, $\left.\mathrm{C}-4_{\alpha}\right), 78.0\left(\mathrm{C}-4_{\beta}\right), 75.6\left(\mathrm{CH}_{2} \mathrm{Bn}_{\alpha}\right), 75.5\left(\mathrm{CH}_{2} \mathrm{Bn}_{\beta}\right), 70.6\left(\mathrm{C}-5_{\beta}\right)$, $69.0\left(\mathrm{~d}, J=20.3 \mathrm{~Hz}, \mathrm{CH}_{2}-\mathrm{CH}_{2} \mathrm{~F}_{\beta}\right), 67.3\left(\mathrm{~d}, J=20.1 \mathrm{~Hz}, \mathrm{CH}_{2}-\right.$ $\left.\mathrm{CH}_{2} \mathrm{~F}_{\alpha}\right), 66.8\left(\mathrm{C}-5_{\alpha}\right), 66.7\left(\mathrm{C}-6_{\alpha}\right), 66.3\left(\mathrm{C}-6_{\beta}\right), 65.5\left(\mathrm{C}-2_{\beta}\right), 62.5$ (C$\left.2_{\alpha}\right)$, 27.5, 27.1 $\left(\mathrm{CH}_{3}{ }^{\mathrm{t}} \mathrm{Bu}_{\alpha, \beta}\right), 23.1\left(\mathrm{C}_{\mathrm{q}}{ }^{\mathrm{t}} \mathrm{Bu}_{\alpha}\right), 22.8\left(\mathrm{C}_{\mathrm{q}}{ }^{\mathrm{t}} \mathrm{Bu}_{\beta}\right), 20.1\left(\mathrm{C}_{\mathrm{q}}\right.$ $\left.{ }^{\mathrm{t}} \mathrm{Bu}_{\alpha}\right)$, $20.1\left(\mathrm{C}_{\mathrm{q}}{ }^{\mathrm{t}} \mathrm{Bu}_{\beta}\right)$. HRMS: $\left[\mathrm{M}-\mathrm{N}_{2}+\mathrm{H}\right]^{+}$calcd for $\mathrm{C}_{23} \mathrm{H}_{37} \mathrm{FNO}_{5} \mathrm{Si} 454.24195$, found 454.24188 .

Methyl 4-O-(2-Azido-3-O-benzyl-2-deoxy-4,6-O-di-tert-butylsilylidene- $\alpha / \beta$-D-glucopyranosyl)-2,3,6-tri-O-benzyl- $\alpha$-D-glucopyranoside (2E). Donor 2 and acceptor 26 were condensed using the general procedure for $\mathrm{Tf}_{2} \mathrm{O} / \mathrm{Ph}_{2} \mathrm{SO}$-mediated glycosylations (for an additional $18 \mathrm{~h}$ at $-40^{\circ} \mathrm{C}$ ) and purified by flash column chromatography (1:0 to 9:1 pentane/EtOAc) to yield glycosylation product $2 \mathrm{E}(72 \mathrm{mg}, 82$ $\mu \mathrm{mol}, 82 \%, \alpha / \beta=1: 3)$ as a colorless oil. $R_{\mathrm{f}} 0.23$ and 0.41 (9:1 pentane/EtOAc). IR (thin film): 654, 696, 735, 768, 827, 962, 1090, $1159,1271,1362,1454,2110,2859,2932$. Data for the $\beta$-anomer: ${ }^{1} \mathrm{H}$ NMR ( $\mathrm{CDCl}_{3}, 400 \mathrm{MHz}, \mathrm{H}-\mathrm{H}$ COSY, HSQC, HMBC): $\delta 7.44-7.39$ $\left(\mathrm{m}, 2 \mathrm{H}, \mathrm{CH}_{\text {arom }}\right), 7.39-7.21\left(\mathrm{~m}, 18 \mathrm{H}, \mathrm{CH}_{\text {arom }}\right), 4.98(\mathrm{~d}, 1 \mathrm{H}, J=10.8$ $\mathrm{Hz}, \mathrm{CHH} \mathrm{Bn}), 4.83-4.74$ (m, 4H, CHH Bn, $\mathrm{CH}_{2} \mathrm{Bn}, \mathrm{CHH} \mathrm{Bn}$ ), 4.68 (d, $1 \mathrm{H}, J=11.9 \mathrm{~Hz}, \mathrm{CHH} \mathrm{Bn}), 4.62(\mathrm{~d}, 1 \mathrm{H}, J=12.2 \mathrm{~Hz}, \mathrm{CHH} \mathrm{Bn})$ $4.59(\mathrm{~d}, 1 \mathrm{H}, J=3.6 \mathrm{~Hz}, \mathrm{H}-1), 4.44(\mathrm{~d}, 1 \mathrm{H}, J=11.9 \mathrm{~Hz}, \mathrm{CHH} \mathrm{Bn})$, $4.23\left(\mathrm{~d}, 1 \mathrm{H}, J=8.0 \mathrm{~Hz}, \mathrm{H}-1^{\prime}\right), 3.97(\mathrm{dd}, 1 \mathrm{H}, J=10.6,3.0 \mathrm{~Hz}, \mathrm{H}-6)$, 3.94-3.73 (m, 5H, H-3, H-4, H-4', H-5, H-6' $), 3.71-3.66$ (m, 1H, H6), 3.55-3.47 (m, 2H, H-2, H-6'), 3.38 (s, 3H, $\mathrm{CH}_{3} \mathrm{OMe}$ ), 3.27-3.21 $\left(\mathrm{m}, 1 \mathrm{H}, \mathrm{H}-2^{\prime}\right), 3.20-3.14\left(\mathrm{~m}, 1 \mathrm{H}, \mathrm{H}-3^{\prime}\right), 3.06(\mathrm{td}, 1 \mathrm{H}, J=9.9,5.1 \mathrm{~Hz}$, $\left.\mathrm{H}-5^{\prime}\right), 1.06$ (s, 9H, $\left.\mathrm{CH}_{3}{ }^{\mathrm{t}} \mathrm{Bu}\right), 0.97$ (s, 9H, $\left.\mathrm{CH}_{3}{ }^{\mathrm{t}} \mathrm{Bu}\right) .{ }^{13} \mathrm{C}-\mathrm{APT}$ NMR $\left(\mathrm{CDCl}_{3}, 101 \mathrm{MHz}, \mathrm{HSQC}, \mathrm{HMBC}\right): \delta$ 139.4, 138.4, 138.1, 137.9 $\left(\mathrm{C}_{\mathrm{q}}\right), 128.5,128.5,128.5,128.4,128.4,128.2,128.1,128.0,128.0$, $127.9,127.4,127.3\left(\mathrm{CH}_{\text {arom }}\right), 101.0\left(\mathrm{C}-1^{\prime}\right), 98.4(\mathrm{C}-1), 82.6\left(\mathrm{C}-3^{\prime}\right)$, 80.2 (C-3), 79.2 (C-2), 78.1 (C-4'), 77.0 (C-4), 75.3, 75.3, 73.6, 73.6 $\left(\mathrm{CH}_{2} \mathrm{Bn}\right), 70.2\left(\mathrm{C}-5^{\prime}\right), 69.7$ (C-5), 68.3 (C-6), $66.2\left(\mathrm{C}-6^{\prime}\right), 66.1$ (C$\left.2^{\prime}\right)$, 55.4 (OMe), 27.6, 27.1 $\left(\mathrm{CH}_{3}{ }^{\mathrm{t}} \mathrm{Bu}\right), 22.7,20.0\left(\mathrm{C}_{\mathrm{q}} \mathrm{tBu}\right)$. Diagnostic peaks for the $\alpha$-anomer: ${ }^{1} \mathrm{H}$ NMR $\left(\mathrm{CDCl}_{3}, 400 \mathrm{MHz}\right): \delta 5.67(\mathrm{~d}, 1 \mathrm{H}$, $J=4.0 \mathrm{~Hz}, \mathrm{H}-1), 5.11(\mathrm{~d}, 1 \mathrm{H}, J=10.6 \mathrm{~Hz}, \mathrm{CHH} \mathrm{Bn}), 5.06(\mathrm{~d}, 1 \mathrm{H}, J=$ $10.6 \mathrm{~Hz}, \mathrm{CHH} \mathrm{Bn}), 4.87$ (d, $1 \mathrm{H}, J=10.6 \mathrm{~Hz}, \mathrm{CHH} \mathrm{Bn}), 4.79(\mathrm{~d}, 1 \mathrm{H}, J$ $=10.6 \mathrm{~Hz}, \mathrm{CHH} \mathrm{Bn}), 4.75(\mathrm{~d}, 1 \mathrm{H}, J=12.0 \mathrm{~Hz}, \mathrm{CHH} \mathrm{Bn}), 4.09(\mathrm{t}, 1 \mathrm{H}$, $J=9.0 \mathrm{~Hz}, \mathrm{H}-3), 3.56(\mathrm{dd}, 1 \mathrm{H}, J=9.6,3.5 \mathrm{~Hz}, \mathrm{H}-2), 3.38\left(\mathrm{~s}, \mathrm{CH}_{3}\right.$ $\mathrm{OMe}), 3.21\left(\mathrm{dd}, 1 \mathrm{H}, \mathrm{J}=10.2,4.0 \mathrm{~Hz}, \mathrm{H}-2^{\prime}\right), 1.06\left(\mathrm{~s}, 9 \mathrm{H}, \mathrm{CH}_{3}{ }^{\mathrm{t}} \mathrm{Bu}\right)$, $1.04\left(\mathrm{~s}, 9 \mathrm{H}, \mathrm{CH}_{3}{ }^{\mathrm{t}} \mathrm{Bu}\right) .{ }^{13} \mathrm{C}-\mathrm{APT}$ NMR $\left(\mathrm{CDCl}_{3}, 101 \mathrm{MHz}\right): \delta 138.8$, $138.3,138.2,138.0,128.6,128.4,128.3,128.1,128.0,128.0,128.0$, $127.9,127.7,127.6,127.4,127.3,97.9,97.7$ (C-1, C-1'), 82.1 (C-3), 
80.6 (C-2), 79.1, 79.0, 75.6, 75.1, 73.7, 73.4, 69.6, 69.2, 67.5, 66.5, 62.3 $\left(\mathrm{C}-2^{\prime}\right), 55.4,27.6,27.2,22.8,20.1$. HRMS: $\left[\mathrm{M}+\mathrm{NH}_{4}\right]^{+}$calcd for $\mathrm{C}_{49} \mathrm{H}_{67} \mathrm{~N}_{4} \mathrm{O}_{10} \mathrm{Si} 899.46210$, found 899.46246 .

Methyl (Methyl 4-O-[2-Azido-3-O-benzyl-2-deoxy-4,6-O-di-tertbutylsilylidene- $\alpha / \beta$-D-glucopyranosyl]-2,3-di-O-benzyl- $\alpha$-D-glucopyranosyl uronate) (2F). Donor 2 and acceptor 27 were condensed using the general procedure for $\mathrm{Tf}_{2} \mathrm{O} / \mathrm{Ph}_{2} \mathrm{SO}$-mediated glycosylations (for an additional $18 \mathrm{~h}$ at $-40{ }^{\circ} \mathrm{C}$ ) and purified by flash column chromatography (1:0 to 9:1 pentane/EtOAc) to yield glycosylation product $2 \mathrm{~F}(69 \mathrm{mg}, 84 \mu \mathrm{mol}, 84 \%, \alpha / \beta=3.3: 1)$ as a white solid. $R_{\mathrm{f}}$ 0.36 and 0.39 (9:1 pentane/EtOAc). IR (thin film): 654, 696, 735, $827,1042,1144,1387,1751,2108,2859,2934$. Data for the $\alpha$ anomer: ${ }^{1} \mathrm{H}$ NMR $\left(\mathrm{CDCl}_{3}, 400 \mathrm{MHz}, \mathrm{H}-\mathrm{H}\right.$ COSY, HSQC, HMBC): $\delta 7.43-7.38\left(\mathrm{~m}, 2 \mathrm{H}, \mathrm{CH}_{\text {arom }}\right), 7.37-7.25\left(\mathrm{~m}, 13 \mathrm{H}, \mathrm{CH}_{\text {arom }}\right), 5.45(\mathrm{~d}$, $\left.1 \mathrm{H}, J=4.1 \mathrm{~Hz}, \mathrm{H}-1^{\prime}\right), 5.07-5.02(\mathrm{~m}, 2 \mathrm{H}, 2 \times \mathrm{CHH} \mathrm{Bn}), 4.90(\mathrm{~d}, 1 \mathrm{H}$, $J=10.6 \mathrm{~Hz}, \mathrm{CHH} \mathrm{Bn}), 4.84-4.78(\mathrm{~m}, 1 \mathrm{H}, \mathrm{CHH} \mathrm{Bn}), 4.75$ (d, 1H, J = $12.0 \mathrm{~Hz}, \mathrm{CHH} \mathrm{Bn}), 4.59$ (d, $1 \mathrm{H}, J=12.2 \mathrm{~Hz}, \mathrm{CHH} \mathrm{Bn}), 4.57$ (d, $1 \mathrm{H}, J$ $=3.5 \mathrm{~Hz}, \mathrm{H}-1), 4.21-4.17(\mathrm{~m}, 1 \mathrm{H}, \mathrm{H}-5), 4.09-4.01(\mathrm{~m}, 3 \mathrm{H}, \mathrm{H}-3, \mathrm{H}-4$, H-6'), 3.91-3.85 (m, 1H, H-4'), 3.83-3.73 (m, 5H, $\mathrm{CH}_{3} \mathrm{CO}_{2} \mathrm{Me}, \mathrm{H}-$ $\left.3^{\prime}, \mathrm{H}-6^{\prime}\right), 3.62\left(\mathrm{td}, 1 \mathrm{H}, J=10.1,5.0 \mathrm{~Hz}, \mathrm{H}-5^{\prime}\right), 3.58-3.53(\mathrm{~m}, 1 \mathrm{H}, \mathrm{H}-$ 2), 3.41 (s, $3 \mathrm{H}, \mathrm{CH}_{3} \mathrm{OMe}$ ), 3.23 (dd, $\left.1 \mathrm{H}, J=10.2,4.1 \mathrm{~Hz}, \mathrm{H}-2^{\prime}\right), 1.07$ $\left(\mathrm{s}, 9 \mathrm{H}, \mathrm{CH}_{3}{ }^{\mathrm{t}} \mathrm{Bu}\right), 1.05\left(\mathrm{~s}, 9 \mathrm{H}, \mathrm{CH}_{3}{ }^{\mathrm{t}} \mathrm{Bu}\right) \cdot{ }^{13} \mathrm{C}-\mathrm{APT}$ NMR $\left(\mathrm{CDCl}_{3}, 101\right.$ $\mathrm{MHz}, \mathrm{HSQC}, \mathrm{HMBC}): \delta 169.2\left(\mathrm{C}=\mathrm{O} \mathrm{CO}_{2} \mathrm{Me}\right), 138.7,138.2,137.8$ $\left(\mathrm{C}_{\mathrm{q}}\right), 128.7,128.5,128.5,128.5,128.3,128.2,128.0,127.7,127.6$ $\left(\mathrm{CH}_{\text {arom }}\right), 98.5,98.4$ (C-1, C-1'), 81.0 (C-3), 79.9 (C-2), 79.0, 79.0 (C-3', C-4'), 76.2 (C-4), 75.5, 75.4, $73.6\left(\mathrm{CH}_{2} \mathrm{Bn}\right), 70.2$ (C-5), 67.0 $\left(\mathrm{C}-5^{\prime}\right), 66.4\left(\mathrm{C}-6^{\prime}\right), 62.4\left(\mathrm{C}-2^{\prime}\right), 55.9(\mathrm{OMe}), 52.9\left(\mathrm{CO}_{2} \mathrm{Me}\right), 27.6$, $27.2\left(\mathrm{CH}_{3}{ }^{\mathrm{t}} \mathrm{Bu}\right), 22.9,20.0\left(\mathrm{C}_{\mathrm{q}}{ }^{\mathrm{t}} \mathrm{Bu}\right)$. Diagnostic peaks for the $\beta$ anomer: ${ }^{1} \mathrm{H}$ NMR $\left(\mathrm{CDCl}_{3}, 400 \mathrm{MHz}\right): \delta 4.98(\mathrm{~d}, 1 \mathrm{H}, J=10.9 \mathrm{~Hz}$, $\mathrm{CHH} \mathrm{Bn}), 4.39$ (d, $\left.1 \mathrm{H}, J=7.7 \mathrm{~Hz}, \mathrm{H}-1^{\prime}\right), 4.02-3.96(\mathrm{~m}, 1 \mathrm{H}), 3.82(\mathrm{~s}$, $3 \mathrm{H}, \mathrm{CH}_{3} \mathrm{CO}_{2} \mathrm{Me}$ ), $3.52(\mathrm{dd}, 1 \mathrm{H}, J=9.5,3.6 \mathrm{~Hz}, \mathrm{H}-2), 1.05(\mathrm{~s}, 9 \mathrm{H}$, $\left.\mathrm{CH}_{3}{ }^{\mathrm{t}} \mathrm{Bu}\right), 0.97\left(\mathrm{~s}, 9 \mathrm{H}, \mathrm{CH}_{3},{ }^{\mathrm{t}} \mathrm{Bu}\right) .{ }^{13} \mathrm{C}-\mathrm{APT}$ NMR $\left(\mathrm{CDCl}_{3}, 101\right.$ $\mathrm{MHz}): \delta 170.2$, 139.1, 138.1, 138.1, 128.6, 128.5, 128.4, 128.3, 128.3, 128.1, 128.0, 127.5, 127.3, 101.9 (C-1'), 98.9 (C-1), 82.5, 79.6, 79.4, 78.8, 78.0, 75.4, 73.9, 70.4, 69.9, 66.1, 55.9, 52.8, 27.5, 27.1, 22.8, 20.0. HRMS: $\left[\mathrm{M}+\mathrm{NH}_{4}\right]^{+}$calcd for $\mathrm{C}_{43} \mathrm{H}_{61} \mathrm{~N}_{4} \mathrm{O}_{11} \mathrm{Si}$ 837.41006, found 837.41042 .

2,2-Difluoroethyl 2-Azido-3-O-benzyl-2-deoxy-4,6-O-di-tert-butylsilylidene- $\alpha / \beta$-D-glucopyranoside (2G). Donor 2 and 2,2-difluoroethanol were condensed using the general procedure for $\mathrm{Tf}_{2} \mathrm{O}$ / $\mathrm{Ph}_{2} \mathrm{SO}$-mediated glycosylations and purified by flash column chromatography (1:0 to $0: 1$ pentane/toluene to $2 \% \mathrm{Et}_{2} \mathrm{O}$ in toluene) to yield glycosylation product $2 \mathrm{G}(38.1 \mathrm{mg}, 76 \mu \mathrm{mol}, 76 \%, \alpha / \beta=$ 2.7:1) in two fractions ( $24.3 \mathrm{mg}$ of $\alpha$ only, $13.8 \mathrm{mg}$ of $\alpha / \beta=0.3: 1$ ) as white solids. $R_{\mathrm{f}} 0.43 \beta, 0.31 \alpha$ (toluene). IR (neat): $654,766,826$, $1070,1474,2108,2860,2934$. Data for the $\alpha$-anomer: $[\alpha]_{\mathrm{D}}^{23}=+35.6^{\circ}$ $\left(c=0.86, \mathrm{CHCl}_{3}\right) .{ }^{1} \mathrm{H}$ NMR $\left(\mathrm{CDCl}_{3}, 400 \mathrm{MHz}, \mathrm{H}-\mathrm{H}\right.$ COSY, HSQC): $\delta 7.45-7.40\left(\mathrm{~m}, 2 \mathrm{H}, \mathrm{CH}_{\text {arom }}\right), 7.39-7.27\left(\mathrm{~m}, 3 \mathrm{H}, \mathrm{CH}_{\text {arom }}\right)$, $5.95\left(\mathrm{tt}, 1 \mathrm{H}, J=55.2,4.1 \mathrm{~Hz}, \mathrm{CHF}_{2}\right), 5.06(\mathrm{~d}, 1 \mathrm{H}, J=10.6 \mathrm{~Hz}, \mathrm{CHH}$ $\mathrm{Bn}), 4.85(\mathrm{~d}, 1 \mathrm{H}, J=3.6 \mathrm{~Hz}, \mathrm{H}-1), 4.82(\mathrm{~d}, 1 \mathrm{H}, J=10.7 \mathrm{~Hz}, \mathrm{CHH}$ $\mathrm{Bn}), 4.13-4.08$ (m, 1H, H-6), 3.98-3.92 (m, 1H, H-3/4), 3.92-3.72 (m, 5H, CH $\left.-\mathrm{CHF}_{2}, \mathrm{H}-3 / 4, \mathrm{H}-5, \mathrm{H}-6\right), 3.35$ (dd, $1 \mathrm{H}, \mathrm{J}=10.1,3.6$ $\mathrm{Hz}, \mathrm{H}-2$ ), 1.09 (s, 9H, $\mathrm{CH}_{3}{ }^{\mathrm{t}} \mathrm{Bu}$ ), 1.03 (s, 9H, $\left.\mathrm{CH}_{3}{ }^{\mathrm{t}} \mathrm{Bu}\right) .{ }^{13} \mathrm{C}-\mathrm{APT}$ NMR ( $\mathrm{CDCl}_{3}, 101 \mathrm{MHz}$, HSQC): $\delta 138.1\left(\mathrm{C}_{\mathrm{q}}\right), 128.6,128.5,128.1$ $\left(\mathrm{CH}_{\text {arom }}\right), 113.8\left(\mathrm{t}, J=241.6 \mathrm{~Hz}, \mathrm{CHF}_{2}\right), 98.7(\mathrm{C}-1), 79.0,78.9$ (C-3, C-4), $75.7\left(\mathrm{CH}_{2} \mathrm{Bn}\right), 67.3\left(\mathrm{t}, J=28.6 \mathrm{~Hz}, \mathrm{CH}_{2}-\mathrm{CHF}_{2}\right), 67.1(\mathrm{C}-5)$, $66.6(\mathrm{C}-6), 62.4(\mathrm{C}-2), 27.5,27.1\left(\mathrm{CH}_{3}{ }^{\mathrm{t}} \mathrm{Bu}\right), 22.8,20.1\left(\mathrm{C}_{\mathrm{q}}{ }^{\mathrm{t}} \mathrm{Bu}\right)$. Data for the $\beta$-anomer: ${ }^{1} \mathrm{H}$ NMR $\left(\mathrm{CDCl}_{3}, 400 \mathrm{MHz}, \mathrm{H}-\mathrm{H}\right.$ COSY, HSQC): $\delta 7.44-7.39\left(\mathrm{~m}, 2 \mathrm{H}, \mathrm{CH}_{\text {arom }}\right), 7.38-7.29\left(\mathrm{~m}, 3 \mathrm{H}, \mathrm{CH}_{\text {arom }}\right)$, $5.92\left(\right.$ tdd $\left.1 \mathrm{H}, J=55.3,5.1,3.4 \mathrm{~Hz}, \mathrm{CHF}_{2}\right), 4.99(\mathrm{~d}, 1 \mathrm{H}, J=10.9 \mathrm{~Hz}$, $\mathrm{CHH} \mathrm{Bn}), 4.81(\mathrm{~d}, 1 \mathrm{H}, J=11.0 \mathrm{~Hz}, \mathrm{CHH} \mathrm{Bn}), 4.35(\mathrm{~s}, 1 \mathrm{H}, J=7.7 \mathrm{~Hz}$, $\mathrm{H}-1$ ), 4.17 (dd, $1 \mathrm{H}, J=10.3,5.0 \mathrm{~Hz}, \mathrm{H}-6), 4.02-3.74\left(\mathrm{~m}, 4 \mathrm{H}, \mathrm{CH}_{2}-\right.$ $\mathrm{CHF}_{2}, \mathrm{H}-4, \mathrm{H}-6$ ), 3.42-3.30 (m, 3H, H-2, H-3, H-5), 1.09 (s, 9H, $\left.\mathrm{CH}_{3}{ }^{\mathrm{t}} \mathrm{Bu}\right), 1.01$ (s, 9H, $\left.\mathrm{CH}_{3},{ }^{\mathrm{t}} \mathrm{Bu}\right) .{ }^{13} \mathrm{C}-\mathrm{APT}$ NMR $\left(\mathrm{CDCl}_{3}, 101 \mathrm{MHz}\right.$, HSQC): $\delta 138.1\left(\mathrm{C}_{\mathrm{q}}\right), 128.5,128.4,128.1\left(\mathrm{CH}_{\text {arom }}\right), 114.1(\mathrm{t}, J=$ $\left.241.4 \mathrm{~Hz}, \mathrm{CHF}_{2}\right), 102.5$ (C-1), $82.2(\mathrm{C}-3), 77.9(\mathrm{C}-4), 75.5\left(\mathrm{CH}_{2}\right.$ $\mathrm{Bn}), 70.7$ (C-5), 68.8 (dd, $\left.J=29.3,28.8 \mathrm{~Hz}, \mathrm{CH}_{2}-\mathrm{CHF}_{2}\right), 66.2$ (C6), 65.4 (C-2), 27.5, $27.1\left(\mathrm{CH}_{3}{ }^{\mathrm{t}} \mathrm{Bu}\right), 22.8,20.1\left(\mathrm{C}_{\mathrm{q}}{ }^{\mathrm{t}} \mathrm{Bu}\right)$. HRMS: [M $\left.-\mathrm{N}_{2}+\mathrm{H}\right]^{+}$calcd for $\mathrm{C}_{23} \mathrm{H}_{36} \mathrm{~F}_{2} \mathrm{NO}_{5} \mathrm{Si}$ 472.23253, found 472.23239.
Methyl 4-O-(2-Azido-3-O-benzyl-2-deoxy-4,6-O-di-tert-butylsilylidene- $\alpha / \beta$-D-glucopyranosyl)-2,3,6-tri-O-benzyl- $\beta$-D-galactopyranoside $(\mathbf{2 H})$. Donor 2 and acceptor 28 were condensed using the general procedure for $\mathrm{Tf}_{2} \mathrm{O} / \mathrm{Ph}_{2} \mathrm{SO}$-mediated glycosylations (for an additional $18 \mathrm{~h}$ at $\left.-40^{\circ} \mathrm{C}\right)$ and purified by flash column chromatography (1:0 to 9:1 pentane/EtOAc) to yield glycosylation product $2 \mathrm{H}(46 \mathrm{mg}, 52$ $\mu \mathrm{mol}, 52 \%, \alpha / \beta=7: 1)$ as a colorless oil. $R_{\mathrm{f}} 0.33$ and 0.51 (9:1 pentane/EtOAc). IR (thin film): 652, 696, 735, 826, 1001, 1036, 1206, $1364,1454,2108,2859,2932$. Data for the $\alpha$-anomer: ${ }^{1} \mathrm{H}$ NMR $\left(\mathrm{CDCl}_{3}, 400 \mathrm{MHz}, \mathrm{H}-\mathrm{H}\right.$ COSY, HSQC, HMBC): $\delta 7.46-7.41(\mathrm{~m}$, $\left.2 \mathrm{H}, \mathrm{CH}_{\text {arom }}\right), 7.41-7.23\left(\mathrm{~m}, 18 \mathrm{H}, \mathrm{CH}_{\text {arom }}\right), 5.10(\mathrm{~d}, 1 \mathrm{H}, J=10.3 \mathrm{~Hz}$, CHH Bn), 4.89-4.81 (m, 3H, CHH Bn, $2 \times \mathrm{CHH} \mathrm{Bn}), 4.79(\mathrm{~d}, 1 \mathrm{H}, J$ $\left.=3.7 \mathrm{~Hz}, \mathrm{H}-1^{\prime}\right), 4.72(\mathrm{~d}, 1 \mathrm{H}, J=10.6 \mathrm{~Hz}, \mathrm{CHH} \mathrm{Bn}), 4.68(\mathrm{~d}, 1 \mathrm{H}, J=$ $13.0 \mathrm{~Hz}, \mathrm{CHH} \mathrm{Bn}$ ), 4.58-4.44 (m, 3H, $\left.\mathrm{CH}_{2} \mathrm{Bn}, \mathrm{H}-5^{\prime}\right), 4.23$ (d, $1 \mathrm{H}, \mathrm{J}$ $=7.6 \mathrm{~Hz}, \mathrm{H}-1), 4.07-3.99(\mathrm{~m}, 2 \mathrm{H}, \mathrm{H}-4, \mathrm{H}-6), 3.99-3.88(\mathrm{~m}, 3 \mathrm{H}, \mathrm{H}-$ $\left.3^{\prime}, \mathrm{H}-4^{\prime}, \mathrm{H}-6^{\prime}\right), 3.76\left(\mathrm{t}, 1 \mathrm{H}, J=10.1 \mathrm{~Hz}, \mathrm{H}-6^{\prime}\right), 3.67-3.58(\mathrm{~m}, 2 \mathrm{H}, \mathrm{H}-$ 2, H-6), 3.56 (s, 3H, $\mathrm{CH}_{3} \mathrm{OMe}$ ), 3.48 (dd, $\left.1 \mathrm{H}, J=8.9,5.5 \mathrm{~Hz}, \mathrm{H}-5\right)$, 3.38 (dd, $1 \mathrm{H}, J=10.0,2.9 \mathrm{~Hz}, \mathrm{H}-3$ ), $3.33(\mathrm{dd}, 1 \mathrm{H}, J=9.7,3.7 \mathrm{~Hz}, \mathrm{H}-$ $\left.2^{\prime}\right), 1.06\left(\mathrm{~s}, 9 \mathrm{H}, \mathrm{CH}_{3}{ }^{\mathrm{t}} \mathrm{Bu}\right), 1.02\left(\mathrm{~s}, 9 \mathrm{H}, \mathrm{CH}_{3}{ }^{\mathrm{t}} \mathrm{Bu}\right) .{ }^{13} \mathrm{C}-\mathrm{APT}$ NMR $\left(\mathrm{CDCl}_{3}, 101 \mathrm{MHz}, \mathrm{HSQC}, \mathrm{HMBC}\right): \delta$ 138.8, 138.4, 138.2, 137.7 $\left(\mathrm{C}_{\mathrm{q}}\right), 128.6,128.6,128.5,128.5,128.5,128.3,128.2,127.9,127.8$ $\left(\mathrm{CH}_{\text {arom }}\right), 105.1$ (C-1), 99.2 (C-1'), 79.9, 79.9 (C-2, C-3'), 79.6, 79.4 (C-3, C-4'), 75.7, 75.6 ( $\left.\mathrm{CH}_{2} \mathrm{Bn}\right), 75.0(\mathrm{C}-4), 73.6\left(\mathrm{CH}_{2} \mathrm{Bn}\right), 72.9$ (C5), $72.6\left(\mathrm{CH}_{2} \mathrm{Bn}\right), 67.1,67.0\left(\mathrm{C}-6, \mathrm{C}-6^{\prime}\right), 66.9\left(\mathrm{C}-5^{\prime}\right), 63.2\left(\mathrm{C}-2^{\prime}\right)$, 57.5 (OMe), 27.5, $27.3\left(\mathrm{CH}_{3}{ }^{\mathrm{t}} \mathrm{Bu}\right), 22.7,20.2\left(\mathrm{C}_{\mathrm{q}}{ }^{\mathrm{t}} \mathrm{Bu}\right)$. Diagnostic peaks for the $\beta$-anomer: ${ }^{1} \mathrm{H}$ NMR $\left(\mathrm{CDCl}_{3}, 400 \mathrm{MHz}\right): \delta 4.94(\mathrm{~d}$, $0.14 \mathrm{H}, J=11.1 \mathrm{~Hz}, \mathrm{CHH} \mathrm{Bn}), 4.27$ (d, $0.14 \mathrm{H}, J=7.7 \mathrm{~Hz}, \mathrm{H}-1), 3.22-$ $3.16(\mathrm{~m}, 0.28 \mathrm{H}), 3.20-3.09(\mathrm{~m}, 2 \mathrm{H}) .{ }^{13} \mathrm{C}-\mathrm{APT} \mathrm{NMR}\left(\mathrm{CDCl}_{3}, 101\right.$ $\mathrm{MHz}): \delta 105.1$ (C-1), 102.0 (C-1'), 82.3, 78.0, 75.4, 75.3, 73.7, 73.7, 73.5, 73.4, 70.4, 69.6, 66.4, 65.6, 57.3, 27.6, 27.2, 22.8, 20.1. HRMS: $\left[\mathrm{M}+\mathrm{NH}_{4}\right]^{+}$calcd for $\mathrm{C}_{49} \mathrm{H}_{67} \mathrm{~N}_{4} \mathrm{O}_{10} \mathrm{Si}$ 899.46210, found 899.46243.

Methyl 2-O-(2-Azido-3-O-benzyl-2-deoxy-4,6-O-di-tert-butylsilylidene- $\alpha$-D-glucopyranosyl)-3-O-benzyl-4,6-O-benzylidene- $\alpha$-D-mannopyranoside (2I). Donor 2 and acceptor 29 were condensed using the general procedure for $\mathrm{Tf}_{2} \mathrm{O} / \mathrm{Ph}_{2} \mathrm{SO}$-mediated glycosylations (for an additional $18 \mathrm{~h}$ at $-40{ }^{\circ} \mathrm{C}$ ) and purified by flash column chromatography (1:0 to $9: 1$ pentane/EtOAc) to yield glycosylation product $2 \mathrm{I}(67 \mathrm{mg}, 85 \mu \mathrm{mol}, 85 \%, \alpha / \beta>20: 1)$ as a white solid. $R_{\mathrm{f}} 0.54$ (9:1 pentane/EtOAc). $[\alpha]_{\mathrm{D}}^{20}=+44.3^{\circ}\left(c=1.34, \mathrm{CHCl}_{3}\right)$. IR (thin film): 696, 827, 937, 1040, 1088, 1130, 1364, 2108, 2859, 2957. Data for the $\alpha$-anomer: ${ }^{1} \mathrm{H} \mathrm{NMR}\left(\mathrm{CDCl}_{3}, 400 \mathrm{MHz}, \mathrm{H}-\mathrm{H}\right.$ COSY, HSQC, HMBC): $\delta 7.54-7.47\left(\mathrm{~m}, 2 \mathrm{H}, \mathrm{CH}_{\text {arom }}\right), 7.46-7.41\left(\mathrm{~m}, 2 \mathrm{H}, \mathrm{CH}_{\text {arom }}\right)$, $7.41-7.22\left(\mathrm{~m}, 11 \mathrm{H}, \mathrm{CH}_{\text {arom }}\right), 5.65(\mathrm{~s}, 1 \mathrm{H}, \mathrm{CHPh}), 5.23(\mathrm{~d}, 1 \mathrm{H}, J=3.6$ $\left.\mathrm{Hz}, \mathrm{H}-1^{\prime}\right), 5.09$ (d, 1H, $\left.J=10.6 \mathrm{~Hz}, \mathrm{CHH} \mathrm{Bn}\right), 4.89-4.82(\mathrm{~m}, 2 \mathrm{H}$, $\mathrm{CHH}$ Bn, CHH Bn), 4.74-4.65 (m, 2H, CHH Bn, H-1), 4.31-4.21 (m, 2H, H-4, H-6), 4.11-3.92 (m, 5H, H-2, H-3, H-3', H-4', H-6'), 3.92-3.76 (m, 4H, H-5, H-5', H-6, H-6'), 3.36 (s, 3H, $\mathrm{CH}_{3} \mathrm{OMe}$ ), $3.27\left(\mathrm{dd}, 1 \mathrm{H}, J=10.0,3.7 \mathrm{~Hz}, \mathrm{H}-2^{\prime}\right), 1.09(\mathrm{~s}, 9 \mathrm{H}), 1.05(\mathrm{~s}, 9 \mathrm{H}) .{ }^{13} \mathrm{C}-$ APT NMR ( $\mathrm{CDCl}_{3}, 101 \mathrm{MHz}$, HSQC, HMBC): $\delta$ 138.6, 138.4, 137.7 $\left(\mathrm{C}_{\mathrm{q}}\right), 129.0,128.5,128.5,128.4,128.3,128.3,128.0,127.6,127.5$, 127.4, 126.2, $126.1\left(\mathrm{CH}_{\text {arom }}\right), 101.7(\mathrm{CHPh}), 101.0(\mathrm{C}-1), 99.4$ (C$1^{\prime}$ ), 79.3 (C-4), 79.1, 78.9 (C-3', C-4'), 76.0, 75.6 (C-2, C-3), 75.6, $73.0\left(\mathrm{CH}_{2} \mathrm{Bn}\right), 69.0(\mathrm{C}-6), 67.2\left(\mathrm{C}-5^{\prime}\right), 66.6\left(\mathrm{C}-6^{\prime}\right), 64.1$ (C-5), 62.6 $\left(\mathrm{C}-2^{\prime}\right)$, 55.2 ( $\left.\mathrm{CH}_{3} \mathrm{OMe}\right), 27.5,27.2\left(\mathrm{CH}_{3}{ }^{\mathrm{t}} \mathrm{Bu}\right), 22.8,20.2\left(\mathrm{C}_{\mathrm{q}}{ }^{\mathrm{t}} \mathrm{Bu}\right)$. Diagnostic peaks for the $\beta$-anomer: ${ }^{1} \mathrm{H}$ NMR $\left(\mathrm{CDCl}_{3}, 400 \mathrm{MHz}\right): \delta$ $5.60(\mathrm{~s}, 1 \mathrm{H}, \mathrm{CHPh}), 4.98(\mathrm{~d}, 1 \mathrm{H}, J=11.3 \mathrm{~Hz}, \mathrm{CHH} \mathrm{Bn}), 4.39$ (d, $1 \mathrm{H}$, $\left.J=8.2 \mathrm{~Hz}, \mathrm{H}-1^{\prime}\right), 3.57-3.48(\mathrm{~m}, 1 \mathrm{H}) .{ }^{13} \mathrm{C}$-APT NMR $\left(\mathrm{CDCl}_{3}, 101\right.$ $\mathrm{MHz}): \delta$ 101.6, 99.6, 81.8, 78.5, 77.8, 76.4, 75.2, 74.4, 72.3, 70.9, 65.7, 55.1, 27.5, 27.1. HRMS: $\left[\mathrm{M}+\mathrm{NH}_{4}\right]^{+}$calcd for $\mathrm{C}_{42} \mathrm{H}_{59} \mathrm{~N}_{4} \mathrm{O}_{10} \mathrm{Si}$ 807.39950, found 807.39931.

2,2,2-Trifluoroethyl 2-Azido-3-O-benzyl-2-deoxy-4,6-O-di-tertbutylsilylidene- $\alpha$-D-glucopyranoside (2J). Donor 2 and 2,2,2trifluoroethanol were condensed using the general procedure for $\mathrm{Tf}_{2} \mathrm{O} / \mathrm{Ph}_{2} \mathrm{SO}$-mediated glycosylations (for an additional $30 \mathrm{~min}$ at -40 ${ }^{\circ} \mathrm{C}$ ) and purified by flash column chromatography (1:0 to $0: 1$ pentane/toluene to $2 \% \mathrm{Et}_{2} \mathrm{O}$ in toluene) to yield glycosylation product 2J (42.4 mg, $82 \mu \mathrm{mol}, 82 \%, \alpha / \beta>20: 1$ ) as a colorless oil. $R_{\mathrm{f}} 0.36$ (toluene). $[\alpha]_{\mathrm{D}}^{23}=+32.6^{\circ}\left(c=1.0, \mathrm{CHCl}_{3}\right)$. IR (neat): 654, 766, 826, 1036, 1082, 1159, 1279, 1472, 2108, 2859, 2930. ${ }^{1} \mathrm{H}$ NMR $\left(\mathrm{CDCl}_{3}\right.$, $400 \mathrm{MHz}, \mathrm{H}-\mathrm{H}$ COSY, HSQC): $\delta 7.45-7.39$ (m, 2H, $\mathrm{CH}_{\text {arom }}$ ), 
$7.38-7.27\left(\mathrm{~m}, 3 \mathrm{H}, \mathrm{CH}_{\text {arom }}\right.$ ), 5.07 (d, $\left.1 \mathrm{H}, J=10.6 \mathrm{~Hz}, \mathrm{CHH} \mathrm{Bn}\right), 4.88$ (d, $1 \mathrm{H}, J=3.6 \mathrm{~Hz}, \mathrm{H}-1), 4.82(\mathrm{~d}, 1 \mathrm{H}, J=10.6 \mathrm{~Hz}, \mathrm{CHH} \mathrm{Bn}$ ), 4.14$4.07(\mathrm{~m}, 1 \mathrm{H}, \mathrm{H}-6), 4.03-3.93\left(\mathrm{~m}, 3 \mathrm{H}, \mathrm{CH}_{2}-\mathrm{CF}_{3}, \mathrm{H}-4\right), 3.92-3.80$ (m, 3H, H-3, H-5, H-6), 3.36 (dd, $1 \mathrm{H}, J=10.0,3.6 \mathrm{~Hz}, \mathrm{H}-2$ ), 1.09 (s, $\left.9 \mathrm{H}, \mathrm{CH}_{3}{ }^{\mathrm{t}} \mathrm{Bu}\right), 1.03\left(\mathrm{~s}, 9 \mathrm{H}, \mathrm{CH}_{3}{ }^{\mathrm{t}} \mathrm{Bu}\right) .{ }^{13} \mathrm{C}-\mathrm{APT}$ NMR $\left(\mathrm{CDCl}_{3}, 101\right.$ $\mathrm{MHz}, \mathrm{HSQC}): \delta 138.1\left(\mathrm{C}_{\mathrm{q}}\right), 128.6,128.5,128.1\left(\mathrm{CH}_{\text {arom }}\right), \delta 123.5(\mathrm{q}$, $J=278.4 \mathrm{~Hz}, \mathrm{CF} 3), 98.8(\mathrm{C}-1), 78.9(\mathrm{C}-3), 78.8(\mathrm{C}-4), 75.7\left(\mathrm{CH}_{2}\right.$ $\mathrm{Bn}), 67.4(\mathrm{C}-5), 66.5(\mathrm{C}-6), 65.2\left(\mathrm{q}, J=35.4 \mathrm{~Hz}, \mathrm{CH}_{2}-\mathrm{CF}_{3}\right), 62.2$ (C-2), 27.5, $27.1\left(\mathrm{CH}_{3}{ }^{\mathrm{t}} \mathrm{Bu}\right), 22.8,20.1\left(\mathrm{C}_{\mathrm{q}}{ }^{\mathrm{t}} \mathrm{Bu}\right)$. HRMS: $\left[\mathrm{M}-\mathrm{N}_{2}+\right.$ $\mathrm{H}]^{+}$calcd for $\mathrm{C}_{23} \mathrm{H}_{35} \mathrm{~F}_{3} \mathrm{NO}_{5} \mathrm{Si}$ 490.22311, found 490.22292.

1,1,1,3,3,3-Hexafluoro-2-propyl 2-azido-3-O-benzyl-2-deoxy-4,6O-di-tert-butylsilylidene- $\alpha$-D-glucopyranoside (2K). Donor 2 and 1,1,1,3,3,3-hexafluoro-2-propanol were condensed using the general procedure for $\mathrm{Tf}_{2} \mathrm{O} / \mathrm{Ph}_{2} \mathrm{SO}$-mediated glycosylations (for an additional $72 \mathrm{~h}$ at $-40{ }^{\circ} \mathrm{C}$ ) and purified by flash column chromatography (4:1 to $0: 1$ pentane/toluene) to yield glycosylation product $2 \mathrm{~K}(20 \mathrm{mg}, 34$ $\mu \mathrm{mol}, 34 \%, \alpha / \beta>20: 1)$ as a white solid. $R_{\mathrm{f}} 0.38\left(9: 1\right.$ pentane $\left./ \mathrm{Et}_{2} \mathrm{O}\right)$. $[\alpha]_{\mathrm{D}}^{20}=+31.2^{\circ}\left(c=0.50, \mathrm{CHCl}_{3}\right)$. IR (thin film): 689, 827, 1030, 1098, $1221,1288,1368,2112,2860,2934 .{ }^{1} \mathrm{H}$ NMR $\left(\mathrm{CDCl}_{3}, 400 \mathrm{MHz}, \mathrm{H}-\right.$ H COSY, HSQC, HMBC, NOESY): $\delta 7.45-7.28\left(\mathrm{~m}, 5 \mathrm{H}, \mathrm{CH}_{\text {arom }}\right)$, 5.12-5.04 (m, 2H, CHH Bn, H-1), $4.83(\mathrm{~d}, 1 \mathrm{H}, J=10.5 \mathrm{~Hz}, \mathrm{CHH}$ $\mathrm{Bn}$ ), 4.40 (hept, $1 \mathrm{H}, J=5.7 \mathrm{~Hz}, \mathrm{CH}$ HFIP), 4.09 (dd, $1 \mathrm{H}, J=9.4,4.0$ $\mathrm{Hz}, \mathrm{H}-6$ ), 4.03-3.91 (m, 2H, H-4, H-5), 3.91-3.83 (m, 2H, H-3, H6), $3.42(\mathrm{dd}, 1 \mathrm{H}, J=10.2,3.8 \mathrm{~Hz}, \mathrm{H}-2), 1.08\left(\mathrm{~s}, 9 \mathrm{H}, \mathrm{CH}_{3}{ }^{\mathrm{t}} \mathrm{Bu}\right), 1.03(\mathrm{~s}$, $\left.9 \mathrm{H}, \mathrm{CH}_{3}{ }^{\mathrm{t}} \mathrm{Bu}\right) \cdot{ }^{13} \mathrm{C}$-APT NMR $\left(\mathrm{CDCl}_{3}, 101 \mathrm{MHz}, \mathrm{HSQC}, \mathrm{HMBC}\right): \delta$ $138.0\left(\mathrm{C}_{\mathrm{q}}\right), 128.6,128.5,128.2\left(\mathrm{CH}_{\text {arom }}\right), 100.4(\mathrm{C}-1), 78.7(\mathrm{C}-3)$, $78.4(\mathrm{C}-4), 75.8\left(\mathrm{CH}_{2} \mathrm{Bn}\right), 73.3(\mathrm{p}, J=33.2 \mathrm{~Hz}), 68.1$ (C-5), 66.1 (C6), 61.9 (C-2), 27.5, 27.0 ( $\left.\mathrm{CH}_{3}{ }^{\mathrm{t}} \mathrm{Bu}\right), 22.8,20.1\left(\mathrm{C}_{\mathrm{q}}{ }^{\mathrm{t}} \mathrm{Bu}\right)$. HRMS: [M $\left.-\mathrm{N}_{2}+\mathrm{H}\right]^{+}$calcd for $\mathrm{C}_{24} \mathrm{H}_{34} \mathrm{~F}_{6} \mathrm{NO}_{5} \mathrm{Si}$ 558.21050, found 558.21009.

Ethyl 2-Azido-3-O-benzyl-4,6-O-benzylidene-2-deoxy- $\beta$-D-glucopyranoside ( $3 A$ ). Donor 3 and ethanol were condensed using the general procedure for $\mathrm{Tf}_{2} \mathrm{O} / \mathrm{Ph}_{2} \mathrm{SO}$-mediated glycosylations and purified by flash column chromatography ( $1: 0$ to $0: 1$ hexane/toluene to $5 \%$ EtOAc in toluene) to yield glycosylation product $3 \mathrm{~A}(34.3 \mathrm{mg}$, $83 \mu \mathrm{mol}, 83 \%, \alpha / \beta<1: 20)$ as a white solid. $R_{\mathrm{f}} 0.58$ (9:1 toluene/ EtOAc). $[\alpha]_{\mathrm{D}}^{23}=-79.6^{\circ}\left(c=0.69, \mathrm{CHCl}_{3}\right)$. IR (neat): 692, 993, 1098, $1186,1267,1365,1452,2111,2878,2979 .{ }^{1} \mathrm{H}$ NMR $\left(\mathrm{CDCl}_{3}, 400\right.$ $\mathrm{MHz}, \mathrm{H}-\mathrm{H}$ COSY, HSQC): $\delta 7.50-7.46\left(\mathrm{~m}, 2 \mathrm{H}, \mathrm{CH}_{\text {arom }}\right), 7.41-7.28$ $\left(\mathrm{m}, 8 \mathrm{H}, \mathrm{CH}_{\text {arom }}\right), 5.57(\mathrm{~s}, 1 \mathrm{H}, \mathrm{CHPh}), 4.91(\mathrm{~d}, 1 \mathrm{H}, J=11.2 \mathrm{~Hz}, \mathrm{CHH}$ $\mathrm{Bn}), 4.79$ (d, $1 \mathrm{H}, J=11.3 \mathrm{~Hz}, \mathrm{CHH} \mathrm{Bn}), 4.37(\mathrm{~d}, 1 \mathrm{H}, J=8.2 \mathrm{~Hz}, \mathrm{H}-$ 1), $4.34(\mathrm{dd}, 1 \mathrm{H}, J=10.6,5.0 \mathrm{~Hz}, \mathrm{H}-6), 3.96(\mathrm{dq}, 1 \mathrm{H}, J=9.7,7.1 \mathrm{~Hz}$, CHH Et), $3.80(\mathrm{t}, 1 \mathrm{H}, J=10.3 \mathrm{~Hz}, \mathrm{H}-6), 3.70(\mathrm{t}, 1 \mathrm{H}, J=9.0 \mathrm{~Hz}, \mathrm{H}-4)$, $3.66(\mathrm{dq}, 1 \mathrm{H}, J=9.7,7.2 \mathrm{~Hz}, \mathrm{CHH} \mathrm{Et}), 3.54(\mathrm{t}, 1 \mathrm{H}, J=9.3 \mathrm{~Hz}, \mathrm{H}-3)$, 3.44 (dd, $1 \mathrm{H}, J=9.5,8.0 \mathrm{~Hz}, \mathrm{H}-2), 3.39(\mathrm{td}, 2 \mathrm{H}, J=9.8,5.0 \mathrm{~Hz}, \mathrm{H}-5)$, $1.29\left(\mathrm{t}, 3 \mathrm{H}, J=7.1 \mathrm{~Hz}, \mathrm{CH}_{3} \mathrm{Et}\right) .{ }^{13} \mathrm{C}$-APT NMR $\left(\mathrm{CDCl}_{3}, 101 \mathrm{MHz}\right.$, HSQC): $\delta 138.0,137.2\left(\mathrm{C}_{\mathrm{q}}\right), 129.2,128.5,128.4,128.3,128.0,126.1$ $\left(\mathrm{CH}_{\text {arom }}\right), 102.5$ (C-1), $101.4(\mathrm{CHPh}), 81.7$ (C-4), 79.1 (C-3), 75.0 $\left(\mathrm{CH}_{2} \mathrm{Bn}\right), 68.7(\mathrm{C}-6), 66.3\left(\mathrm{CH}_{2} \mathrm{Et}\right), 66.3,66.2(\mathrm{C}-2, \mathrm{C}-5), 15.2$ $\left(\mathrm{CH}_{3} \mathrm{Et}\right) .{ }^{13} \mathrm{C}-\mathrm{HMBC}-\mathrm{GATED}$ NMR $\left(\mathrm{CDCl}_{3}, 101 \mathrm{MHz}\right): \delta 102.5$ $\left(J_{\mathrm{C} 1, \mathrm{H} 1}=161 \mathrm{~Hz}, \mathrm{C}-1\right)$. HRMS: $\left[\mathrm{M}+\mathrm{NH}_{4}\right]^{+}$calcd for $\mathrm{C}_{22} \mathrm{H}_{29} \mathrm{~N}_{4} \mathrm{O}_{5}$ 429.21325, found 429.21321.

Cyclohexyl 2-Azido-3-O-benzyl-4,6-O-benzylidene-2-deoxy- $\beta$-Dglucopyranoside (3B). Donor 3 and cyclohexanol were condensed using the general procedure for $\mathrm{Tf}_{2} \mathrm{O} / \mathrm{Ph}_{2} \mathrm{SO}$-mediated glycosylations and purified by flash column chromatography (1:1 to $0: 1$ hexane/ toluene to $5 \%$ EtOAc in toluene) to yield glycosylation product $3 \mathrm{~B}$ (43 mg, $93 \mu \mathrm{mol}, 93 \%, \alpha / \beta<1: 20$ ) as a white solid. $R_{\mathrm{f}} 0.23$ (toluene). $[\alpha]_{\mathrm{D}}^{23}=-60.5^{\circ}(c=0.86, \mathrm{DCM})$. IR (neat): 696, 748, 998, 1092, 1275, $1365,1452,2108,2858,2933$. Data for the $\beta$-anomer: ${ }^{1} \mathrm{H}$ NMR $\left(\mathrm{CDCl}_{3}, 400 \mathrm{MHz}, \mathrm{H}-\mathrm{H}\right.$ COSY, HSQC $): \delta 7.50-7.44(\mathrm{~m}, 2 \mathrm{H}$, $\left.\mathrm{CH}_{\text {arom }}\right), 7.42-7.27\left(\mathrm{~m}, 8 \mathrm{H}, \mathrm{CH}_{\text {arom }}\right), 5.56(\mathrm{~s}, 1 \mathrm{H}, \mathrm{CHPh}), 4.90(\mathrm{~d}$, $1 \mathrm{H}, J=11.4 \mathrm{~Hz}, \mathrm{CHH} \mathrm{Bn}), 4.79(\mathrm{~d}, 1 \mathrm{H}, J=11.4 \mathrm{~Hz}, \mathrm{CHH} \mathrm{Bn}), 4.47$ (d, $1 \mathrm{H}, J=7.8 \mathrm{~Hz}, \mathrm{H}-1), 4.32(\mathrm{dd}, 1 \mathrm{H}, J=10.5,5.0 \mathrm{~Hz}, \mathrm{H}-6), 3.79(\mathrm{t}$, $1 \mathrm{H}, J=10.3 \mathrm{~Hz}, \mathrm{H}-6), 3.74-3.64(\mathrm{~m}, 2 \mathrm{H}, \mathrm{H}-4, \mathrm{CH} \mathrm{Cyc}), 3.50(\mathrm{t}, 1 \mathrm{H}$, $J=9.2 \mathrm{~Hz}, \mathrm{H}-3), 3.44$ (dd, $1 \mathrm{H}, J=9.6,7.8 \mathrm{~Hz}, \mathrm{H}-2), 3.36(\mathrm{td}, 1 \mathrm{H}, J=$ 9.7, $5.0 \mathrm{~Hz}, \mathrm{H}-5), 1.99-1.87\left(\mathrm{~m}, 2 \mathrm{H}, \mathrm{CH}_{2} \mathrm{Cyc}\right), 1.82-1.72(\mathrm{~m}, 2 \mathrm{H}, J$ $\left.=15.2,4.4 \mathrm{~Hz}, \mathrm{CH}_{2} \mathrm{Cyc}\right), 1.56-1.37\left(\mathrm{~m}, 3 \mathrm{H}, \mathrm{CH}_{2} \mathrm{Cyc}\right), 1.36-1.20$ $\left(\mathrm{m}, 3 \mathrm{H}, \mathrm{CH}_{2} \mathrm{Cyc}\right) .{ }^{13} \mathrm{C}-\mathrm{APT}$ NMR ( $\left.\mathrm{CDCl}_{3}, 101 \mathrm{MHz}, \mathrm{HSQC}\right): \delta$ 138.0, $137.3\left(\mathrm{C}_{\mathrm{q}}\right), 129.1,128.5,128.4,128.3,127.9,126.1\left(\mathrm{CH}_{\text {arom }}\right)$, 101.4 (CHPh), 101.0 (C-1), 81.6 (C-4), 79.0 (C-3), 78.5 (CH Суc),
$75.0\left(\mathrm{CH}_{2} \mathrm{Bn}\right), 68.8$ (C-6), 66.5 (C-2), 66.3 (C-5), 33.6, 31.8, 25.6, 24.1, $23.9\left(\mathrm{CH}_{2} \mathrm{Cyc}\right)$. Diagnostic peaks for the $\alpha$-anomer: ${ }^{1} \mathrm{H}$ NMR $\left(\mathrm{CDCl}_{3}, 400 \mathrm{MHz}\right): \delta 5.59(\mathrm{~s}, 0.04 \mathrm{H}, \mathrm{CHPh}), 5.03(\mathrm{~d}, 0.04 \mathrm{H}, J=3.7$ $\mathrm{Hz}, \mathrm{H}-1), 4.12(\mathrm{t}, 0.04 \mathrm{H}, J=9.5 \mathrm{~Hz}, \mathrm{H}-3), 4.00(\mathrm{td}, 0.04 \mathrm{H}, J=9.9,4.8$ $\mathrm{Hz}, \mathrm{H}-5$ ), 3.28 (dd, $0.04 \mathrm{H}, J=10.0,3.7 \mathrm{~Hz}, \mathrm{H}-2) .{ }^{13} \mathrm{C}-\mathrm{APT}$ NMR $\left(\mathrm{CDCl}_{3}, 101 \mathrm{MHz}\right): \delta 101.4(\mathrm{CHPh}), 97.1(\mathrm{C}-1), 76.0(\mathrm{C}-3), 63.8$ (C-2), 62.9 (C-5). HRMS: $\left[\mathrm{M}+\mathrm{NH}_{4}\right]^{+}$calcd for $\mathrm{C}_{26} \mathrm{H}_{35} \mathrm{~N}_{4} \mathrm{O}_{5}$ 483.26020, found 483.25991.

Methyl 6-O-(2-Azido-3-O-benzyl-4,6-O-benzylidene-2-deoxy- $\beta$-Dglucopyranosyl)-2,3,4-tri-O-benzyl- $\alpha$-D-glucopyranoside (3C). Donor 3 and acceptor $\mathbf{2 5}$ were condensed using the general procedure for $\mathrm{Tf}_{2} \mathrm{O} / \mathrm{Ph}_{2} \mathrm{SO}$-mediated glycosylations (for an additional $18 \mathrm{~h}$ at $-40{ }^{\circ} \mathrm{C}$ ) and purified by flash column chromatography (19:1 to $4: 1$ pentane/EtOAc) to yield glycosylation product $3 \mathrm{C}(73.7 \mathrm{mg}, 89 \mu \mathrm{mol}$, $89 \%, \alpha / \beta<1: 20)$ as a white solid. $R_{\mathrm{f}} 0.42$ (4:1 pentane/EtOAc). Spectroscopic data were in accord with those reported previously. ${ }^{38}$ $[\alpha]_{\mathrm{D}}^{23}=-32.2^{\circ}\left(c=1.0, \mathrm{CHCl}_{3}\right)$. IR (neat): 696, 737, 999, 1028, 1070, 1090, 1277, 1362, 1497, 2108, 2876, 2926. ${ }^{1} \mathrm{H}$ NMR $\left(\mathrm{CDCl}_{3}, 400\right.$ $\mathrm{MHz}, \mathrm{H}-\mathrm{H}$ COSY, HSQC, HMBC): $\delta 7.47$ (dd, $2 \mathrm{H}, J=7.3,2.5 \mathrm{~Hz}$, $\left.\mathrm{CH}_{\text {arom }}\right), 7.42-7.25\left(\mathrm{~m}, 23 \mathrm{H}, \mathrm{CH}_{\text {arom }}\right), 5.55(\mathrm{~s}, 1 \mathrm{H}, \mathrm{CHPh}), 4.99(\mathrm{~d}$, $1 \mathrm{H}, J=10.9 \mathrm{~Hz}, \mathrm{CHH} 3-\mathrm{OBn}), 4.95(\mathrm{~d}, 1 \mathrm{H}, J=11.2 \mathrm{~Hz}, \mathrm{CHH} 4-$ $\mathrm{OBn}), 4.91\left(\mathrm{~d}, 1 \mathrm{H}, J=11.2 \mathrm{~Hz}, \mathrm{CHH} 3^{\prime}-\mathrm{OBn}\right), 4.85-4.76(\mathrm{~m}, 3 \mathrm{H}$, $\left.\mathrm{CHH} 3-\mathrm{OBn}, \mathrm{CHH} 2-\mathrm{OBn}, \mathrm{CHH} 3^{\prime}-\mathrm{OBn}\right), 4.70-4.63(\mathrm{~m}, 2 \mathrm{H}, \mathrm{CHH}$ 4-OBn, CHH 2-OBn), 4.61 (d, $1 \mathrm{H}, J=3.6 \mathrm{~Hz}, \mathrm{H}-1), 4.30$ (dd, $1 \mathrm{H}, J=$ $\left.10.5,5.0 \mathrm{~Hz}, \mathrm{H}-6^{\prime}\right), 4.23\left(\mathrm{~d}, 1 \mathrm{H}, J=7.9 \mathrm{~Hz}, \mathrm{H}-\mathrm{1}^{\prime}\right), 4.07$ (d, $1 \mathrm{H}, J=8.9$ Hz, H-6), 4.00 (t, 1H, J = 9.3 Hz, H-3), 3.81-3.72 (m, 3H, H-5, H-6, $\left.\mathrm{H}-6^{\prime}\right), 3.69\left(\mathrm{t}, 1 \mathrm{H}, J=9.1 \mathrm{~Hz}, \mathrm{H}-4^{\prime}\right), 3.60(\mathrm{t}, 1 \mathrm{H}, J=9.3 \mathrm{~Hz}, \mathrm{H}-4)$, 3.59-3.46 (m, 3H, H-2, H-2', H-3'), 3.37 (s, 3H, $\left.\mathrm{CH}_{3} \mathrm{OMe}\right), 3.36-$ $3.29\left(\mathrm{~m}, 1 \mathrm{H}, \mathrm{H}-5^{\prime}\right) .{ }^{13} \mathrm{C}-\mathrm{APT}$ NMR $\left(\mathrm{CDCl}_{3}, 101 \mathrm{MHz}, \mathrm{HSQC}\right.$, HMBC): $\delta 138.8,138.5,138.2,137.8,137.2\left(\mathrm{C}_{\mathrm{q}}\right), 129.2,128.6,128.5$, $128.5,128.4,128.3,128.3,128.1,128.1,128.0,127.9,127.9,127.7$, $126.1\left(\mathrm{CH}_{\text {arom }}\right), 102.4\left(\mathrm{C}-1^{\prime}\right), 101.4(\mathrm{CHPh}), 98.4(\mathrm{C}-1), 82.2(\mathrm{C}-3)$, 81.5 (C-4'), 79.8 (C-2), 79.3 (C-3'), 77.6 (C-4), $75.9\left(\mathrm{CH}_{2}\right.$ 3-OBn), 75.0, $75.0\left(\mathrm{CH}_{2} 3^{\prime}-\mathrm{OBn}, 4-\mathrm{OBn}\right), 73.6\left(\mathrm{CH}_{2} 2-\mathrm{OBn}\right), 69.6(\mathrm{C}-5)$, 68.7, 68.6 (C-6, C-6'), 66.3 (C-5'), 66.1 (C-2'), 55.4 (OMe). HRMS: $\left[\mathrm{M}+\mathrm{NH}_{4}\right]^{+}$calcd for $\mathrm{C}_{48} \mathrm{H}_{55} \mathrm{~N}_{4} \mathrm{O}_{10} 847.39127$, found 847.39224.

2-Fluoroethyl 2-Azido-3-O-benzyl-4,6-O-benzylidene-2-deoxy- $\alpha$ / $\beta$-D-glucopyranoside (3D). Donor 3 and 2-fluoroethanol were condensed using the general procedure for $\mathrm{Tf}_{2} \mathrm{O} / \mathrm{Ph}_{2} \mathrm{SO}$-mediated glycosylations and purified by flash column chromatography (1:0 to $0: 1$ pentane/toluene to $5 \%$ EtOAc in toluene) to yield glycosylation product 3D (38.5 mg, $90 \mu \mathrm{mol}, 90 \%, \alpha / \beta=1: 6.7)$ as a white solid. $R_{\mathrm{f}}$ 0.40 (19:1 toluene/EtOAc). IR (neat): 696, 748, 996, 1028, 1072, $1091,1174,1276,1368,1454,2108,2873,2917$. Data for the $\beta$ anomer: ${ }^{1} \mathrm{H}$ NMR $\left(\mathrm{CDCl}_{3}, 400 \mathrm{MHz}, \mathrm{H}-\mathrm{H}\right.$ COSY, HSQC): $\delta 7.50-$ $7.46\left(\mathrm{~m}, 2 \mathrm{H}, \mathrm{CH}_{\text {arom }}\right), 7.41-7.36\left(\mathrm{~m}, 5 \mathrm{H}, \mathrm{CH}_{\text {arom }}\right), 7.36-7.25(\mathrm{~m}, 3 \mathrm{H}$, $\left.\mathrm{CH}_{\text {arom }}\right), 5.57(\mathrm{~s}, 1 \mathrm{H}, \mathrm{CHPh}), 4.91(\mathrm{~d}, 1 \mathrm{H}, J=11.2 \mathrm{~Hz}, \mathrm{CHH} \mathrm{Bn})$, $4.79(\mathrm{~d}, 1 \mathrm{H}, J=11.3 \mathrm{~Hz}, \mathrm{CHH} \mathrm{Bn}), 4.69-4.64(\mathrm{~m}, 1 \mathrm{H}, \mathrm{CHHF}), 4.55$ (dt, $1 \mathrm{H}, J=4.6,2.9 \mathrm{~Hz}, \mathrm{CHHF}), 4.42(\mathrm{~d}, 1 \mathrm{H}, J=7.9 \mathrm{~Hz}, \mathrm{H}-1), 4.34$ (dd, $1 \mathrm{H}, J=10.5,5.0 \mathrm{~Hz}, \mathrm{H}-6$ ), 4.11 (ddd, $0.5 \mathrm{H}, J=12.2,4.8,2.9 \mathrm{~Hz}$, $\mathrm{CH}-\mathrm{CFH}_{2}$ ), 4.03 (ddd, $0.5 \mathrm{H}, J=12.2,4.7,3.0 \mathrm{~Hz}, \mathrm{CHH}-\mathrm{CFH}_{2}$ ), 3.92 (ddd, $0.5 \mathrm{H}, J=12.2,5.9,3.2 \mathrm{~Hz}, \mathrm{CHH}-\mathrm{CFH}_{2}$ ), 3.86 (ddd, $\left.0.5 \mathrm{H}, J=12.2,6.0,3.3 \mathrm{~Hz}, \mathrm{CHH}-\mathrm{CFH}_{2}\right), 3.80(\mathrm{t}, 1 \mathrm{H}, J=10.3 \mathrm{~Hz}$, H-6), $3.71(\mathrm{t}, 1 \mathrm{H}, J=9.2 \mathrm{~Hz}, \mathrm{H}-4), 3.56(\mathrm{t}, 1 \mathrm{H}, J=9.2 \mathrm{~Hz}, \mathrm{H}-3), 3.48$ $(\mathrm{dd}, 1 \mathrm{H}, J=9.5,7.9 \mathrm{~Hz}, \mathrm{H}-2), 3.39(\mathrm{td}, 1 \mathrm{H}, J=9.7,4.9 \mathrm{~Hz}, \mathrm{H}-5) .{ }^{13} \mathrm{C}-$ APT NMR ( $\mathrm{CDCl}_{3}, 101 \mathrm{MHz}$, HSQC): $\delta 137.8,137.1\left(\mathrm{C}_{\mathrm{q}}\right), 129.2$, $128.5,128.4,128.3,128.0,126.1\left(\mathrm{CH}_{\text {arom }}\right), 102.7$ (C-1), 101.4 $(\mathrm{CHPh}), 82.6\left(\mathrm{~d}, J=170.1 \mathrm{~Hz}, \mathrm{CFH}_{2}\right), 81.5(\mathrm{C}-4), 79.0(\mathrm{C}-3), 75.1$ $\left(\mathrm{CH}_{2} \mathrm{Bn}\right), 69.3\left(\mathrm{~d}, \mathrm{~J}=20.1 \mathrm{~Hz}, \mathrm{CH}_{2}-\mathrm{CFH}_{2}\right), 68.6$ (C-6), 66.3 (C-5), $66.1(\mathrm{C}-2) .{ }^{13} \mathrm{C}$-HMBC-GATED NMR $\left(\mathrm{CDCl}_{3}, 101 \mathrm{MHz}\right): \delta 102.7$ $\left(J_{\mathrm{C} 1, \mathrm{H} 1}=162 \mathrm{~Hz}, \mathrm{C}-1\right)$. Diagnostic peaks for the $\alpha$-anomer: ${ }^{1} \mathrm{H}$ NMR $\left(\mathrm{CDCl}_{3}, 400 \mathrm{MHz}, \mathrm{H}-\mathrm{H}\right.$ COSY, HSQC $): \delta 5.58(\mathrm{~s}, 0.15 \mathrm{H}, \mathrm{CHPh})$, $4.96(\mathrm{~d}, 0.15 \mathrm{H}, J=10.9 \mathrm{~Hz}, \mathrm{CHH} \mathrm{Bn}), 4.95(\mathrm{~d}, 0.15 \mathrm{H}, J=3.7 \mathrm{~Hz}, \mathrm{H}-$ 1), $4.81(\mathrm{~d}, 0.15 \mathrm{H}, J=11.0 \mathrm{~Hz}, \mathrm{CHH} \mathrm{Bn}), 4.29$ (dd, $0.15 \mathrm{H}, J=10.2$, $4.9 \mathrm{~Hz}, \mathrm{H}-6) .{ }^{13} \mathrm{C}-\mathrm{APT}$ NMR $\left(\mathrm{CDCl}_{3}, 101 \mathrm{MHz}, \mathrm{HSQC}\right): \delta 101.5$ (CHPh), 98.8 (C-1), 82.8 (C-4), 76.2 (C-3), $75.2\left(\mathrm{CH}_{2} \mathrm{Bn}\right), 68.9$ (C6), 63.0, 62.9 (C-2, C-5). ${ }^{13} \mathrm{C}-\mathrm{HMBC}$-GATED NMR $\left(\mathrm{CDCl}_{3}, 101\right.$ $\mathrm{MHz}): \delta 98.8\left(\mathrm{~J}_{\mathrm{C} 1, \mathrm{H} 1}=172 \mathrm{~Hz}, \mathrm{C}-1\right)$. HRMS: $\left[\mathrm{M}+\mathrm{NH}_{4}\right]^{+}$calcd for $\mathrm{C}_{22} \mathrm{H}_{28} \mathrm{FN}_{4} \mathrm{O}_{5}$ 447.20382, found 447.20355. 
Methyl 4-O-(2-Azido-3-O-benzyl-4,6-O-benzylidene-2-deoxy- $\alpha / \beta$ D-glucopyranosyl)-2,3,6-tri-O-benzyl- $\alpha$-D-glucopyranoside (3E). Donor 3 and acceptor 26 were condensed using the general procedure for $\mathrm{Tf}_{2} \mathrm{O} / \mathrm{Ph}_{2} \mathrm{SO}$-mediated glycosylations (for an additional $18 \mathrm{~h}$ at $-40{ }^{\circ} \mathrm{C}$ ) and purified by flash column chromatography (19:1 to $4: 1$ pentane/EtOAc) to yield glycosylation product $3 \mathrm{E}(73.3 \mathrm{mg}, 88 \mu \mathrm{mol}$, $88 \%, \alpha / \beta=1: 7)$ as a white solid. $R_{\mathrm{f}} 0.51 \alpha, 0.43 \beta$ (4:1 pentane/ EtOAc). IR (neat): 696, 737, 1049, 1092, 1362, 1454, $2110,2868$. Data for the $\beta$-anomer: ${ }^{1} \mathrm{H}$ NMR $\left(\mathrm{CDCl}_{3}, 400 \mathrm{MHz}, \mathrm{H}-\mathrm{H}\right.$ COSY, HSQC, TOCSY): $\delta 7.68-7.60\left(\mathrm{~m}, 2 \mathrm{H}, \mathrm{CH}_{\text {arom }}\right), 7.52-7.18(\mathrm{~m}, 23 \mathrm{H}$, $\mathrm{CH}_{\text {arom }}$ ), $5.47(\mathrm{~s}, 1 \mathrm{H}, \mathrm{CHPh}), 4.89$ (d, $\left.1 \mathrm{H}, J=11.2 \mathrm{~Hz}, \mathrm{CHH} \mathrm{Bn}\right)$, $4.87(\mathrm{~d}, 1 \mathrm{H}, J=10.9 \mathrm{~Hz}, \mathrm{CHH} \mathrm{Bn}), 4.81(\mathrm{~d}, 1 \mathrm{H}, J=10.9 \mathrm{~Hz}, \mathrm{CHH}$ $\mathrm{Bn}), 4.78(\mathrm{~d}, 1 \mathrm{H}, J=12.2 \mathrm{~Hz}, \mathrm{CHH} \mathrm{Bn}), 4.75(\mathrm{~d}, 1 \mathrm{H}, J=11.2 \mathrm{~Hz}$, $\mathrm{CH} H \mathrm{Bn}), 4.71(\mathrm{~d}, 1 \mathrm{H}, J=12.0 \mathrm{~Hz}, \mathrm{CHH} \mathrm{Bn}), 4.63(\mathrm{~d}, 1 \mathrm{H}, J=12.1$ $\mathrm{Hz}, \mathrm{CHH} \mathrm{Bn}$ ), 4.60 (d, $1 \mathrm{H}, J=3.7 \mathrm{~Hz}, \mathrm{H}-1), 4.41(\mathrm{~d}, 1 \mathrm{H}, J=12.0 \mathrm{~Hz}$, CHH Bn), 4.19 (d, 1H, J = 7.6 Hz, H-1'), 4.11 (dd, $1 \mathrm{H}, J=10.6,5.0$ $\left.\mathrm{Hz}, \mathrm{H}-6^{\prime}\right), 4.00-3.90$ (m, 2H, H-4, H-6), 3.85 (t, $1 \mathrm{H}, J=9.3 \mathrm{~Hz}, \mathrm{H}-3$ ), $3.75(\mathrm{dt}, 1 \mathrm{H}, J=9.8,2.4 \mathrm{~Hz}, \mathrm{H}-5), 3.69(\mathrm{dd}, 1 \mathrm{H}, J=10.8,1.9 \mathrm{~Hz}, \mathrm{H}-$ 6), $3.56\left(\mathrm{t}, 1 \mathrm{H}, J=9.0 \mathrm{~Hz}, \mathrm{H}-4^{\prime}\right), 3.51(\mathrm{dd}, 1 \mathrm{H}, J=9.5,3.7 \mathrm{~Hz}, \mathrm{H}-2)$, 3.45-3.38 (m, 4H, H-6', $\left.\mathrm{CH}_{3} \mathrm{OMe}\right)$, 3.36-3.27 (m, 2H, H-2', H-3'), $3.00\left(\mathrm{td}, 1 \mathrm{H}, J=9.8,5.0 \mathrm{~Hz}, \mathrm{H}-5^{\prime}\right) .{ }^{13} \mathrm{C}-\mathrm{APT}$ NMR $\left(\mathrm{CDCl}_{3}, 101\right.$ MHz, HSQC): $\delta$ 139.3, 138.3, 137.8, 137.8, $137.3\left(\mathrm{C}_{\mathrm{q}}\right), 131.1,129.4$, 128.6, 128.4, 128.3, 128.2, 128.2, 128.1, 128.1, 127.9, 127.9, 127.6, 126.0, $124.8\left(\mathrm{CH}_{\text {arom }}\right), 101.3,101.2\left(\mathrm{CHPh}, \mathrm{C}-1^{\prime}\right), 98.4(\mathrm{C}-1), 81.7$ (C-4'), 80.1 (C-3), 79.2 (C-3'), 79.0 (C-2), 76.9 (C-4), 75.4, 74.7, 73.6, 73.5 ( $\left.\mathrm{CH}_{2} \mathrm{Bn}\right), 69.7(\mathrm{C}-5), 68.6\left(\mathrm{C}-6^{\prime}\right), 68.0(\mathrm{C}-6), 66.6\left(\mathrm{C}-2^{\prime}\right)$, $65.8\left(\mathrm{C}-5^{\prime}\right), 55.4(\mathrm{OMe})$. Diagnostic peaks for the $\alpha$-anomer: ${ }^{1} \mathrm{H}$ NMR $\left(\mathrm{CDCl}_{3}, 400 \mathrm{MHz}\right): \delta 5.71\left(\mathrm{~d}, 1 \mathrm{H}, J=4.0 \mathrm{~Hz}, \mathrm{H}-1^{\prime}\right), 5.53(\mathrm{~s}$, $1 \mathrm{H}, \mathrm{CHPh}), 5.11(\mathrm{~d}, 1 \mathrm{H}, J=10.7 \mathrm{~Hz}, \mathrm{CHH} \mathrm{Bn}), 4.95(\mathrm{~d}, 1 \mathrm{H}, J=10.9$ $\mathrm{Hz}, \mathrm{CHH} \mathrm{Bn}) .{ }^{13} \mathrm{C}-\mathrm{APT}$ NMR $\left(\mathrm{CDCl}_{3}, 101 \mathrm{MHz}\right): \delta$ 98.1, 97.8, 82.7, 82.1, 80.5, 76.2, 75.1, 73.3, 73.0, 69.4, 69.1, 68.7, 63.4, 62.9. HRMS: $[\mathrm{M}+\mathrm{Na}]^{+}$calcd for $\mathrm{C}_{48} \mathrm{H}_{51} \mathrm{~N}_{3} \mathrm{O}_{10} \mathrm{Na} 852.34667$, found 852.34668.

Methyl (Methyl 4-O-[2-Azido-3-O-benzyl-4,6-O-benzylidene-2deoxy- $\alpha / \beta$-D-glucopyranosyl]-2,3-di-O-benzyl- $\alpha$-D-glucopyranosyl uronate) (3F). Donor 3 and acceptor 27 were condensed using the general procedure for $\mathrm{Tf}_{2} \mathrm{O} / \mathrm{Ph}_{2} \mathrm{SO}$-mediated glycosylations (for an additional $18 \mathrm{~h}$ at $-40{ }^{\circ} \mathrm{C}$ ) and purified by flash column chromatography (19:1 to $4: 1$ pentane/EtOAc) to yield glycosylation product $3 \mathrm{~F}(71.8 \mathrm{mg}, 93 \mu \mathrm{mol}, 93 \%, \alpha / \beta=1.1: 1)$ as a white solid. $R_{\mathrm{f}}$ 0.54 (4:1 pentane/EtOAc). Spectroscopic data were in accord with those reported previously. ${ }^{21}$ IR (neat): 696, 735, 914, 989, 1028, 1045, $1090,1267,1369,1454,1749,2108,2870,2916$. Data reported for a 1:1 mixture of anomers: ${ }^{1} \mathrm{H}$ NMR $\left(\mathrm{CDCl}_{3}, 400 \mathrm{MHz}, \mathrm{H}-\mathrm{H}\right.$ COSY, HSQC, HMBC): $\delta 7.48-7.41\left(\mathrm{~m}, 4 \mathrm{H}, \mathrm{CH}_{\text {arom }}\right), 7.41-7.24(\mathrm{~m}, 36 \mathrm{H}$, $\left.\mathrm{CH}_{\text {arom }}\right), 5.53\left(\mathrm{~s}, 1 \mathrm{H}, \mathrm{CHPh}{ }_{\alpha}\right), 5.51\left(\mathrm{~d}, 1 \mathrm{H}, J=3.9 \mathrm{~Hz}, \mathrm{H}_{-1}{ }^{\prime}{ }_{\alpha}\right), 5.47(\mathrm{~s}$, $1 \mathrm{H}, \mathrm{CHPh}$ ) $5.04(\mathrm{~d}, 1 \mathrm{H}, J=10.5 \mathrm{~Hz}, \mathrm{CHH} \mathrm{Bn}), 4.94(\mathrm{~d}, 1 \mathrm{H}, J=$ $11.0 \mathrm{~Hz}, \mathrm{CHH} \mathrm{Bn}), 4.91-4.82(\mathrm{~m}, 4 \mathrm{H}, 2 \times \mathrm{CHH} \mathrm{Bn}, 2 \times \mathrm{CHH} \mathrm{Bn})$, 4.81-4.72 (m, 4H, $2 \times \mathrm{CHH}$ Bn, $2 \times \mathrm{CHH} \mathrm{Bn}), 4.64-4.58(\mathrm{~m}, 2 \mathrm{H}, 2$ $\times \mathrm{CHH} \mathrm{Bn}), 4.57$ (d, $\left.2 \mathrm{H}, J=3.5 \mathrm{~Hz}, \mathrm{H}-1_{\alpha, \beta}\right), 4.43(\mathrm{~d}, 1 \mathrm{H}, J=8.1 \mathrm{~Hz}$, $\left.\mathrm{H}-1^{\prime}{ }_{\beta}\right), 4.26\left(\mathrm{dd}, 1 \mathrm{H}, J=10.3,4.8 \mathrm{~Hz}, \mathrm{H}-6^{\prime}{ }_{\alpha}\right), 4.24-4.19(\mathrm{~m}, 2 \mathrm{H}, \mathrm{H}-$ $\left.5_{\alpha}, \mathrm{H}-5_{\beta}\right), 4.09-3.99\left(\mathrm{~m}, 4 \mathrm{H}, \mathrm{H}-3_{\beta}, \mathrm{H}-4_{\alpha}, \mathrm{H}-4_{\beta}, \mathrm{H}-6^{\prime}{ }_{\beta}\right), 3.97(\mathrm{t}, 1 \mathrm{H}, \mathrm{J}=$ $\left.9.5 \mathrm{~Hz}, \mathrm{H}-3^{\prime}{ }_{\alpha}\right), 3.89\left(\mathrm{t}, 1 \mathrm{H}, J=9.2 \mathrm{~Hz}, \mathrm{H}-3_{\alpha}\right), 3.82\left(\mathrm{~s}, 3 \mathrm{H}, \mathrm{CH}_{3}\right.$ $\mathrm{CO}_{2} \mathrm{Me}$ ), 3.81 (s, $\left.3 \mathrm{H}, \mathrm{CH}_{3} \mathrm{CO}_{2} \mathrm{Me}\right), 3.72-3.56\left(\mathrm{~m}, 4 \mathrm{H}, \mathrm{H}-2_{\beta}, \mathrm{H}-4^{\prime}{ }_{\alpha}\right.$, $\left.\mathrm{H}-4^{\prime}{ }_{\beta}, \mathrm{H}-6^{\prime}{ }_{\alpha}\right), 3.56-3.46\left(\mathrm{~m}, 3 \mathrm{H}, \mathrm{H}-2_{\alpha}, \mathrm{H}-3^{\prime}{ }_{\beta}, \mathrm{H}-5^{\prime}{ }_{\alpha}\right), 3.46-3.38$ (m, $\left.7 \mathrm{H}, 2 \times \mathrm{CH}_{3} \mathrm{OMe}, \mathrm{H}-6^{\prime}{ }_{\beta}\right), 3.36-3.29\left(\mathrm{~m}, 2 \mathrm{H}, \mathrm{H}-2^{\prime}{ }_{\alpha}, \mathrm{H}-2^{\prime}{ }_{\beta}\right), 3.26(\mathrm{td}$, $\left.1 \mathrm{H}, J=9.7,5.0 \mathrm{~Hz}, \mathrm{H}-5^{\prime}{ }_{\beta}\right) .{ }^{13} \mathrm{C}-\mathrm{APT} \mathrm{NMR}\left(\mathrm{CDCl}_{3}, 101 \mathrm{MHz}\right.$, HSQC, HMBC): $\delta$ 170.0, $170.0\left(\mathrm{C}=\mathrm{O} \mathrm{CO}_{2} \mathrm{Me}\right), 139.1,138.5,138.0$, $137.9,137.9,137.8,137.4,137.2\left(\mathrm{C}_{\mathrm{q}}\right), 129.2,129.1,128.7,128.6$, $128.5,128.5,128.4,128.3,128.3,128.3,128.3,128.2,128.1,128.0$, 128.0, 127.8, 127.7, 127.5, 127.4, 126.1, $126.1\left(\mathrm{CH}_{\text {arom }}\right), 102.3\left(\mathrm{C}-1^{\prime}{ }_{\beta}\right)$, 101.4 $\left(\mathrm{CHPh}_{\beta}\right), 101.3\left(\mathrm{CHPh}_{\alpha}\right), 98.9,98.6\left(\mathrm{C}-1_{\alpha}, \mathrm{C}-1_{\beta}\right), 98.5\left(\mathrm{C}-1^{\prime}\right.$ $\left.{ }_{\alpha}\right), 82.4\left(\mathrm{C}-4^{\prime}{ }_{\alpha}\right), 81.6\left(\mathrm{C}-4^{\prime}{ }_{\beta}\right), 81.1\left(\mathrm{C}-3_{\beta}\right), 79.6\left(\mathrm{C}-2_{\beta}, \mathrm{C}-4_{\beta}\right), 79.5(\mathrm{C}-$ $\left.3_{\alpha}\right), 79.4\left(\mathrm{C}-3^{\prime}{ }_{\beta}\right), 78.7\left(\mathrm{C}-2_{\alpha}\right), 76.3\left(\mathrm{C}-3^{\prime}{ }_{\alpha}\right), 75.6\left(\mathrm{CH}_{2} \mathrm{Bn}\right), 75.5(\mathrm{C}-$ $\left.4_{\alpha}\right), 75.1,75.0,73.9,73.7\left(\mathrm{CH}_{2} \mathrm{Bn}\right), 70.0,69.9\left(\mathrm{C}-5_{\alpha}, \mathrm{C}-5_{\beta}\right), 68.5,68.5$ $\left(\mathrm{C}-6_{\alpha}, \mathrm{C}-6_{\beta}\right), 66.7\left(\mathrm{C}-2^{\prime}{ }_{\beta}\right), 66.2\left(\mathrm{C}-5^{\prime}{ }_{\beta}\right), 63.0\left(\mathrm{C}-5^{\prime}{ }_{\alpha}\right), 62.8\left(\mathrm{C}-2^{\prime}{ }_{\alpha}\right)$, 55.9, $55.9(\mathrm{OMe}), 53.0,52.8\left(\mathrm{CO}_{2} \mathrm{Me}\right)$. HRMS: $\left[\mathrm{M}+\mathrm{NH}_{4}\right]^{+}$calcd for $\mathrm{C}_{42} \mathrm{H}_{49} \mathrm{~N}_{4} \mathrm{O}_{11}$ 785.33923, found 785.34007.

2,2-Difluoroethyl 2-Azido-3-O-benzyl-4,6-O-benzylidene-2deoxy- $\alpha / \beta$-D-glucopyranoside (3G). Donor 3 and 2,2-difluoroethanol were condensed using the general procedure for $\mathrm{Tf}_{2} \mathrm{O} / \mathrm{Ph}_{2} \mathrm{SO}$ - mediated glycosylations and purified by flash column chromatography (1:0 to $0: 1$ pentane/toluene to $5 \%$ EtOAc in toluene) to yield glycosylation product $3 \mathrm{G}(28.8 \mathrm{mg}, 64 \mu \mathrm{mol}, 64 \%, \alpha / \beta=2.9: 1)$ as a white solid. $R_{\mathrm{f}} 0.15$ and 0.18 (toluene). IR (neat): 698, 747, 998, 1070, $1093,1372,1454,2109,2867,2934$. Data reported for a $1: 0.35$ mixture of anomers: ${ }^{1} \mathrm{H}$ NMR $\left(\mathrm{CDCl}_{3}, 400 \mathrm{MHz}, \mathrm{H}-\mathrm{H}\right.$ COSY, HSQC): $\delta 7.52-7.45\left(\mathrm{~m}, 2.70 \mathrm{H}, \mathrm{CH}_{\text {arom }}\right), 7.43-7.26(\mathrm{~m}, 10.80 \mathrm{H}$, $\left.\mathrm{CH}_{\text {arom }}\right), 5.95\left(\mathrm{tt}, 1 \mathrm{H}, J=55.2,4.2 \mathrm{~Hz}, \mathrm{CF}_{2} \mathrm{H}_{\alpha}\right), 5.94(\mathrm{tt}, 0.35 \mathrm{H}, J=$ 55.3, $\left.3.8 \mathrm{~Hz}, \mathrm{CF}_{2} \mathrm{H}_{\beta}\right), 5.58\left(\mathrm{~s}, 1 \mathrm{H}, \mathrm{CHPh}_{\alpha}\right), 5.57\left(\mathrm{~s}, 0.35 \mathrm{H}, \mathrm{CHPh}_{\beta}\right)$, $4.96\left(\mathrm{~d}, 1 \mathrm{H}, J=10.9 \mathrm{~Hz}, \mathrm{CHH} \mathrm{Bn}{ }_{\alpha}\right), 4.93\left(\mathrm{~d}, 1 \mathrm{H}, J=3.9 \mathrm{~Hz}, \mathrm{H}-1_{\alpha}\right)$, $4.92(\mathrm{~d}, 0.35 \mathrm{H}, J=11.3 \mathrm{~Hz}, \mathrm{CHH} \mathrm{Bn})), 4.80(\mathrm{~d}, 1 \mathrm{H}, J=11.0 \mathrm{~Hz}$, $\left.\mathrm{CHH} \mathrm{Bn}_{\alpha}\right), 4.79(\mathrm{~d}, 0.35 \mathrm{H}, J=11.3 \mathrm{~Hz}, \mathrm{CHH} \mathrm{Bn} \beta), 4.40$ (d, $0.35 \mathrm{H}, J$ $\left.=7.9 \mathrm{~Hz}, \mathrm{H}-1_{\beta}\right), 4.34\left(\mathrm{dd}, 0.35 \mathrm{H}, J=10.5,5.0 \mathrm{~Hz}, \mathrm{H}-6_{\beta}\right), 4.29(\mathrm{dd}$, $\left.1 \mathrm{H}, J=10.2,4.8 \mathrm{~Hz}, \mathrm{H}-6_{\alpha}\right), 4.08\left(\mathrm{t}, 1 \mathrm{H}, J=9.5 \mathrm{~Hz}, \mathrm{H}-3_{\alpha}\right), 4.02-3.67$ $\left(\mathrm{m}, 6.4 \mathrm{H}, \mathrm{H}-4_{\alpha}, \mathrm{H}-4_{\beta}, \mathrm{H}-5_{\alpha}, \mathrm{H}-6_{\alpha}, \mathrm{H}-6_{\beta}, \mathrm{CH}_{2}-\mathrm{CF}_{2} \mathrm{H}_{\alpha}, \mathrm{CH}_{2}-\right.$ $\left.\mathrm{CF}_{2} \mathrm{H}_{\beta}\right), 3.56\left(\mathrm{t}, 0.35 \mathrm{H}, J=9.2 \mathrm{~Hz}, \mathrm{H}-3_{\beta}\right), 3.50-3.35\left(\mathrm{~m}, 1.70 \mathrm{H}, \mathrm{H}-2_{\alpha}\right.$, $\left.\mathrm{H}-2_{\beta}, \mathrm{H}-5_{\beta}\right) .{ }^{13} \mathrm{C}-\mathrm{APT}$ NMR $\left(\mathrm{CDCl}_{3}, 101 \mathrm{MHz}, \mathrm{HSQC}\right): \delta 137.8$, $137.8,137.1,137.1\left(\mathrm{C}_{\mathrm{q}}\right), 129.4,129.3,129.1,128.6,128.5,128.5$, $128.4,128.1,126.1\left(\mathrm{CH}_{\text {arom }}\right), 114.0\left(\mathrm{t}, J=241.5 \mathrm{~Hz}, \mathrm{CF}_{2} \mathrm{H}_{\beta}\right), 113.8(\mathrm{t}$, $\left.J=241.6 \mathrm{~Hz}, \mathrm{CF}_{2} \mathrm{H}_{\alpha}\right), 102.8\left(\mathrm{C}-1_{\beta}\right), 101.6\left(\mathrm{CHPh}_{\alpha}\right), 101.5\left(\mathrm{CHPh}_{\beta}\right)$, $99.3\left(\mathrm{C}-1_{\alpha}\right), 82.6\left(\mathrm{C}-4_{\alpha}\right), 81.4\left(\mathrm{C}-4_{\beta}\right), 78.9\left(\mathrm{C}-3_{\beta}\right), 76.0\left(\mathrm{C}-3_{\alpha}\right), 75.3$ $\left(\mathrm{CH}_{2} \mathrm{Bn}_{\alpha}\right), 75.1\left(\mathrm{CH}_{2} \mathrm{Bn}\right), 69.0\left(\mathrm{t}, J=29.0 \mathrm{~Hz}, \mathrm{CH}_{2}-\mathrm{CF}_{2} \mathrm{H}_{\beta}\right), 68.8$ (C-6), $68.5(\mathrm{C}-6), 67.5\left(\mathrm{t}, J=28.7 \mathrm{~Hz}, \mathrm{CH}_{2}-\mathrm{CF}_{2} \mathrm{H}_{\alpha}\right), 66.4\left(\mathrm{C}-5_{\beta}\right)$, $66.0\left(\mathrm{C}-2_{\beta}\right), 63.3\left(\mathrm{C}-5_{\alpha}\right), 62.9\left(\mathrm{C}-2_{\alpha}\right)$. HRMS: $[\mathrm{M}+\mathrm{H}]^{+}$calcd for $\mathrm{C}_{22} \mathrm{H}_{24} \mathrm{~F}_{2} \mathrm{~N}_{3} \mathrm{O}_{5} 448.16785$, found 448.16761 .

Methyl 4-O-(2-Azido-3-O-benzyl-4,6-O-benzylidene-2-deoxy- $\alpha / \beta$ D-glucopyranosyl)-2,3,6-tri-O-benzyl- $\beta$-D-galactopyranoside (3H). Donor $\mathbf{3}$ and acceptor $\mathbf{2 8}$ were condensed using the general procedure for $\mathrm{Tf}_{2} \mathrm{O} / \mathrm{Ph}_{2} \mathrm{SO}$-mediated glycosylations (for an additional $18 \mathrm{~h}$ at $-40{ }^{\circ} \mathrm{C}$ ) and purified by flash column chromatography (19:1 to $4: 1$ pentane/EtOAc) to yield glycosylation product $3 \mathrm{H}(62.2 \mathrm{mg}, 75$ $\mu \mathrm{mol}, 75 \%, \alpha / \beta=9: 1)$ as a white solid. $R_{\mathrm{f}} 0.52$ (4:1 pentane/EtOAc). IR (neat): 696, 735, 995, 1030, 1072, 1090, 1368, 1454, 1497, 2106, 2862, 2920. Data for the $\alpha$-anomer: ${ }^{1} \mathrm{H}$ NMR $\left(\mathrm{CDCl}_{3}, 400 \mathrm{MHz}, \mathrm{H}-\right.$ H COSY, HSQC, HMBC): $\delta 7.50-7.46\left(\mathrm{~m}, 2 \mathrm{H}, \mathrm{CH}_{\text {arom }}\right), 7.42-7.25$ $\left(\mathrm{m}, 23 \mathrm{H}, \mathrm{CH}_{\text {arom }}\right), 5.51(\mathrm{~s}, 1 \mathrm{H}, \mathrm{CHPh}), 4.98(\mathrm{~d}, 1 \mathrm{H}, J=10.7 \mathrm{~Hz}$, CHH 3'-OBn), 4.90 (d, 1H, J = $11.0 \mathrm{~Hz}, \mathrm{CHH} 2-\mathrm{OBn}), 4.88(\mathrm{~d}, 1 \mathrm{H}, J$ $\left.=3.7 \mathrm{~Hz}, \mathrm{H}-1^{\prime}\right), 4.84-4.76\left(\mathrm{~m}, 3 \mathrm{H}, \mathrm{CHH} 2-\mathrm{OBn}, \mathrm{CHH} 3^{\prime}-\mathrm{OBn}\right.$, $\mathrm{CHH} 3-\mathrm{OBn}), 4.69$ (d, 1H, $J=12.4 \mathrm{~Hz}, \mathrm{CHH} 3-\mathrm{OBn}), 4.59-4.51$ (m, $\left.2 \mathrm{H}, \mathrm{CH}_{2} 6-\mathrm{OBn}\right), 4.30\left(\mathrm{td}, 1 \mathrm{H}, J=10.1,4.9 \mathrm{~Hz}, \mathrm{H}-5^{\prime}\right), 4.25(\mathrm{~d}, 1 \mathrm{H}, J$ $=7.6 \mathrm{~Hz}, \mathrm{H}-1), 4.14-4.07\left(\mathrm{~m}, 2 \mathrm{H}, \mathrm{H}-3^{\prime}, \mathrm{H}-4\right), 4.03(\mathrm{t}, 1 \mathrm{H}, J=8.9 \mathrm{~Hz}$, H-6), 3.80 (dd, $\left.1 \mathrm{H}, J=10.2,4.9 \mathrm{~Hz}, \mathrm{H}-6^{\prime}\right), 3.70-3.60(\mathrm{~m}, 3 \mathrm{H}, \mathrm{H}-2$, H-4', H-6), 3.55 (s, 3H, $\mathrm{CH}_{3} \mathrm{OMe}$ ), 3.54-3.48 (m, 2H, H-5, H-6'), 3.44-3.36 (m, 2H, H-2', H-3). ${ }^{13} \mathrm{C}-\mathrm{APT}$ NMR $\left(\mathrm{CDCl}_{3}, 101 \mathrm{MHz}\right.$, HSQC, HMBC): $\delta$ 138.7, 138.3, 138.1, 137.7, $137.6\left(\mathrm{C}_{\mathrm{q}}\right), 129.0$, $128.6,128.5,128.5,128.4,128.3,128.3,128.3,128.2,128.0,127.7$, 127.6, 126.1 ( $\left.\mathrm{CH}_{\text {arom }}\right), 105.3(\mathrm{C}-1), 101.2(\mathrm{CHPh}), 99.4\left(\mathrm{C}-1^{\prime}\right), 83.1$ (C-4'), 80.1 (C-3), 78.9 (C-2), $77.0\left(\mathrm{C}-3^{\prime}\right), 75.3\left(\mathrm{CH}_{2} 3^{\prime}-\mathrm{OBn}\right), 75.1$ $\left(\mathrm{CH}_{2}\right.$ 2-OBn), 74.7 (C-4), $73.6\left(\mathrm{CH}_{2} 6-\mathrm{OBn}\right), 73.0\left(\mathrm{CH}_{2} 3-\mathrm{OBn}\right)$, 72.9 (C-5), 68.9 (C-6'), 67.1 (C-6), $63.8\left(\mathrm{C}-2^{\prime}\right), 62.9$ (C-5'), 57.4 (OMe). Diagnostic peaks for the $\beta$-anomer: ${ }^{1} \mathrm{H}$ NMR $\left(\mathrm{CDCl}_{3}, 400\right.$ $\mathrm{MHz}): \delta 5.54(\mathrm{~s}, 1 \mathrm{H}, \mathrm{CHPh}), 3.92(\mathrm{dd}, 1 \mathrm{H}, J=9.7,7.7 \mathrm{~Hz}, \mathrm{H}-2)$, $3.76-3.71(\mathrm{~m}, 1 \mathrm{H}, \mathrm{H}-6), 3.22\left(\mathrm{td}, 1 \mathrm{H}, J=9.7,4.8 \mathrm{~Hz}, \mathrm{H}-5^{\prime}\right) .{ }^{13} \mathrm{C}-\mathrm{APT}$ NMR $\left(\mathrm{CDCl}_{3}, 101 \mathrm{MHz}\right): \delta 105.1$ (C-1), $102.6\left(\mathrm{C}-1^{\prime}\right), 101.4,81.5$, 81.3, 79.5, 79.0, 74.0, 73.4, 69.4, 68.6, 66.3, 66.0, 57.4. HRMS: [M + $\left.\mathrm{NH}_{4}\right]^{+}$calcd for $\mathrm{C}_{48} \mathrm{H}_{55} \mathrm{~N}_{4} \mathrm{O}_{10} 847.39127$, found 847.39206.

Methyl 2-O-(2-Azido-3-O-benzyl-4,6-O-benzylidene-2-deoxy- $\alpha / \beta$ D-glucopyranosyl)-3-O-benzyl-4,6-O-benzylidene- $\alpha$-D-mannopyranoside (3I). Donor 3 and acceptor 29 were condensed using the general procedure for $\mathrm{Tf}_{2} \mathrm{O} / \mathrm{Ph}_{2} \mathrm{SO}$-mediated glycosylations (for an additional $18 \mathrm{~h}$ at $-40{ }^{\circ} \mathrm{C}$ ) and purified by flash column chromatography (19:1 to $4: 1$ pentane/EtOAc) to yield glycosylation product $3 \mathrm{I}(54.7 \mathrm{mg}, 74 \mu \mathrm{mol}, 74 \%, \alpha / \beta=9: 1)$ as a white solid. $R_{\mathrm{f}}$ 0.74 (7/2 pentane/EtOAc). IR (neat): 696, 746, 997, 1036, 1074, $1090,1128,1371,1454,2106,2862,2922$. Data for the $\alpha$-anomer: ${ }^{1} \mathrm{H}$ NMR ( $\mathrm{CDCl}_{3}, 400 \mathrm{MHz}, \mathrm{H}-\mathrm{H}$ COSY, HSQC, HMBC): $\delta 7.54-7.47$ (m, $\left.4 \mathrm{H}, \mathrm{CH}_{\text {arom }}\right), 7.44-7.25\left(\mathrm{~m}, 16 \mathrm{H}, \mathrm{CH}_{\text {arom }}\right), 5.66(\mathrm{~s}, 1 \mathrm{H}, \mathrm{CHPh})$, $5.60(\mathrm{~s}, 1 \mathrm{H}, \mathrm{CHPh}), 5.39\left(\mathrm{~d}, 1 \mathrm{H}, J=3.7 \mathrm{~Hz}, \mathrm{H}-1^{\prime}\right), 5.00(\mathrm{~d}, 1 \mathrm{H}, J=$ $11.0 \mathrm{~Hz}, \mathrm{CHH} \mathrm{Bn}), 4.90$ (d, $1 \mathrm{H}, J=12.2 \mathrm{~Hz}, \mathrm{CHH} \mathrm{Bn}), 4.84(\mathrm{~d}, 1 \mathrm{H}, J$ $=10.9 \mathrm{~Hz}, \mathrm{CHH} \mathrm{Bn}), 4.73-4.66$ (m, 2H, H-1, CHH Bn), 4.34-4.24 
(m, 3H, H-4, H-6, H-6') 4.17 (dd, 1H, $J=10.2,9.0 \mathrm{~Hz}, \mathrm{H}-3^{\prime}$ ), 4.09 (dd, $1 \mathrm{H}, J=3.1,1.7 \mathrm{~Hz}, \mathrm{H}-2), 4.00(\mathrm{dd}, 1 \mathrm{H}, J=9.9,3.1 \mathrm{~Hz}, \mathrm{H}-3)$, 3.95-3.86 (m, 2H, H-5', H-6), 3.83-3.70 (m, 3H, H-4', H-5, H-6'), $3.36\left(\mathrm{~s}, 3 \mathrm{H}, \mathrm{CH}_{3} \mathrm{OMe}\right), 3.32\left(\mathrm{dd}, 1 \mathrm{H}, J=10.2,3.8 \mathrm{~Hz}, \mathrm{H}-2^{\prime}\right) .{ }^{13} \mathrm{C}-$ APT NMR ( $\mathrm{CDCl}_{3}, 101 \mathrm{MHz}$, HSQC, HMBC): $\delta 138.7,138.0,137.7$, $137.2\left(\mathrm{C}_{\mathrm{q}}\right), 129.2,129.0,128.6,128.5,128.4,128.3,128.2,128.0$, 127.6, 127.4, 126.2, $126.0\left(\mathrm{CH}_{\text {arom }}\right), 101.7(\mathrm{CHPh}), 101.5\left(\mathrm{CHPh}^{\prime}\right)$, 101.0 (C-1), 99.8 (C-1' $), 82.9\left(\mathrm{C}-4^{\prime}\right), 79.4(\mathrm{C}-4), 75.9$ (C-3), $75.6(\mathrm{C}-$ $\left.3^{\prime}\right)$, 75.5 (C-2), 75.3, $73.3\left(\mathrm{CH}_{2} \mathrm{Bn}\right), 69.0,68.9\left(\mathrm{C}-6, \mathrm{C}-6^{\prime}\right), 64.1$ (C5), $63.3\left(\mathrm{C}-5^{\prime}\right), 63.0\left(\mathrm{C}-2^{\prime}\right), 55.0(\mathrm{OMe})$. Diagnostic peaks for the $\beta$ anomer: ${ }^{1} \mathrm{H}$ NMR $\left(\mathrm{CDCl}_{3}, 400 \mathrm{MHz}\right): \delta 4.43(\mathrm{~d}, 0.1 \mathrm{H}, J=8.0 \mathrm{~Hz}, \mathrm{H}-$ $\left.1^{\prime}\right), 3.62\left(\mathrm{dd}, 0.1 \mathrm{H}, J=9.6,8.0 \mathrm{~Hz}, \mathrm{H}-2^{\prime}\right), 3.51(\mathrm{t}, 0.1 \mathrm{H}, J=9.3 \mathrm{~Hz}, \mathrm{H}-$ 4). ${ }^{13} \mathrm{C}$-APT NMR $\left(\mathrm{CDCl}_{3}, 101 \mathrm{MHz}\right): \delta 102.0\left(\mathrm{C}-1^{\prime}\right), 78.5(\mathrm{C}-4)$, $66.4\left(\mathrm{C}-2^{\prime}\right)$. HRMS: $\left[\mathrm{M}+\mathrm{NH}_{4}\right]^{+}$calcd for $\mathrm{C}_{41} \mathrm{H}_{47} \mathrm{~N}_{4} \mathrm{O}_{10} 755.32867$, found 755.32921 .

2,2,2-Trifluoroethyl 2-Azido-3-O-benzyl-4,6-O-benzylidene-2deoxy- $\alpha$-D-glucopyranoside (3J). Donor 3 and 2,2,2-trifluoroethanol were condensed using the general procedure for $\mathrm{Tf}_{2} \mathrm{O} / \mathrm{Ph}_{2} \mathrm{SO}$ mediated glycosylations (for an additional $1 \mathrm{~h}$ at $-40{ }^{\circ} \mathrm{C}$ ) and purified by flash column chromatography (1:0 to $0: 1$ pentane/toluene to $5 \%$ EtOAc in toluene) to yield glycosylation product $3 \mathrm{~J} \mathrm{(44} \mathrm{mg,} 94$ $\mu \mathrm{mol}, 94 \%, \alpha / \beta>20: 1)$ as a colorless oil. $R_{\mathrm{f}} 0.24$ (toluene). $[\alpha]_{\mathrm{D}}^{23}=$ $+25.9^{\circ}(c=0.88, \mathrm{DCM})$. IR (neat): 697, 747, 1001, 1034, 1090, 1165, $1279,1373,2108,2865,2934$. Data for the $\alpha$-anomer: ${ }^{1} \mathrm{H}$ NMR $\left(\mathrm{CDCl}_{3}, 400 \mathrm{MHz}, \mathrm{H}-\mathrm{H}\right.$ COSY, HSQC): $\delta 7.51-7.48(\mathrm{~m}, 2 \mathrm{H}$, $\left.\mathrm{CH}_{\text {arom }}\right), 7.42-7.28\left(\mathrm{~m}, 8 \mathrm{H}, \mathrm{CH}_{\text {arom }}\right), 5.58(\mathrm{~s}, 1 \mathrm{H}, \mathrm{CHPh}), 4.99-4.94$ (m, 2H, CHH Bn, H-1), 4.80 (d, 1H, J = 10.9 Hz, CHH Bn), 4.29 (dd, $1 \mathrm{H}, J=10.2,4.8 \mathrm{~Hz}, \mathrm{H}-6), 4.10$ (dd, $1 \mathrm{H}, J=10.0,9.0 \mathrm{~Hz}, \mathrm{H}-3), 3.98$ (qd, $\left.2 \mathrm{H}, J=8.5,3.1 \mathrm{~Hz}, \mathrm{CH}_{2}-\mathrm{CF}_{3}\right), 3.91(\mathrm{td}, 1 \mathrm{H}, J=9.9,4.8 \mathrm{~Hz}, \mathrm{H}-$ 5), $3.79-3.70$ (m, $2 \mathrm{H}, \mathrm{H}-4, \mathrm{H}-6), 3.43$ (dd, $1 \mathrm{H}, J=10.0,3.7 \mathrm{~Hz}, \mathrm{H}-2$ ). ${ }^{13} \mathrm{C}$-APT NMR $\left(\mathrm{CDCl}_{3}, 101 \mathrm{MHz}\right.$, HSQC): $\delta$ 137.8, 137.1 (C), $129.3,128.6,128.5,128.3,128.1,126.1\left(\mathrm{CH}_{\text {arom }}\right), 123.5(\mathrm{q}, J=278.5$ $\mathrm{Hz}), 101.6(\mathrm{CHPh}), 99.4(\mathrm{C}-1), 82.5(\mathrm{C}-4), 75.9(\mathrm{C}-3), 75.3\left(\mathrm{CH}_{2}\right.$ $\mathrm{Bn}), 68.7(\mathrm{C}-6), 65.4(\mathrm{q}, J=35.4 \mathrm{~Hz}), 63.5(\mathrm{C}-5), 62.7(\mathrm{C}-2) .{ }^{13} \mathrm{C}-$ HMBC-GATED NMR $\left(\mathrm{CDCl}_{3}, 101 \mathrm{MHz}\right): \delta 102.5\left(J_{\mathrm{Cl}, \mathrm{H} 1}=173 \mathrm{~Hz}\right.$, $\mathrm{C}-1)$. Diagnostic peaks for the $\beta$-anomer: ${ }^{1} \mathrm{H}$ NMR $\left(\mathrm{CDCl}_{3}, 400\right.$ $\mathrm{MHz}): \delta 4.44(\mathrm{~d}, 0.03 \mathrm{H}, J=7.9 \mathrm{~Hz}, \mathrm{H}-1), 4.34(\mathrm{dd}, 0.03 \mathrm{H}, J=10.8$, $5.3 \mathrm{~Hz}, \mathrm{H}-6), 3.56(\mathrm{t}, 0.03 \mathrm{H}, J=9.2 \mathrm{~Hz}), 3.48(\mathrm{dd}, 0.03 \mathrm{H}, J=10.0,7.9$ $\mathrm{Hz}, \mathrm{H}-2) .{ }^{13} \mathrm{C}-\mathrm{HMBC}-\mathrm{GATED}$ NMR $\left(\mathrm{CDCl}_{3}, 101 \mathrm{MHz}\right): \delta 102.4$ $\left(J_{\mathrm{C}-\mathrm{H}}=150 \mathrm{~Hz}, \mathrm{C}-1\right)$. HRMS: $\left[\mathrm{M}+\mathrm{NH}_{4}\right]^{+}$calcd for $\mathrm{C}_{22} \mathrm{H}_{26} \mathrm{~F}_{3} \mathrm{~N}_{4} \mathrm{O}_{5}$ 483.18498, found 483.18463 .

1,1,1,3,3,3-Hexafluoro-2-propyl 2-Azido-3-O-benzyl-4,6-O-benzylidene-2-deoxy- $\alpha$-D-glucopyranoside (3K). Donor 3 and $1,1,1,3,3,3-$ hexafluoroisopropanol were condensed using the general procedure for $\mathrm{Tf}_{2} \mathrm{O} / \mathrm{Ph}_{2} \mathrm{SO}$-mediated glycosylations (for an additional $72 \mathrm{~h}$ at $\left.-40{ }^{\circ} \mathrm{C}\right)$ and purified by flash column chromatography (1:0 to $9: 1$ pentane/EtOAc) to yield glycosylation product $3 \mathrm{~K}(28.1 \mathrm{mg}, 53 \mu \mathrm{mol}$, $53 \%, \alpha / \beta>20: 1)$ as a colorless oil. $[\alpha]_{\mathrm{D}}^{23}=+25.8^{\circ}\left(c=0.5, \mathrm{CHCl}_{3}\right)$. IR (neat): 689, 748, 999, 1092, 1196, 1219, 1287, 1368, 2108, 2868, 2928. ${ }^{1} \mathrm{H}$ NMR $\left(\mathrm{CDCl}_{3}, 400 \mathrm{MHz}, \mathrm{H}-\mathrm{H}\right.$ COSY, HSQC $): \delta$ 7.54$7.45\left(\mathrm{~m}, 2 \mathrm{H}, \mathrm{CH}_{\text {arom }}\right), 7.44-7.27\left(\mathrm{~m}, 8 \mathrm{H}, \mathrm{CH}_{\text {arom }}\right), 5.59(\mathrm{~s}, 1 \mathrm{H}$, $\mathrm{CHPh}), 5.13(\mathrm{~d}, 1 \mathrm{H}, J=3.9 \mathrm{~Hz}, \mathrm{H}-1), 4.99$ (d, $1 \mathrm{H}, J=10.8 \mathrm{~Hz}, \mathrm{CHH}$ $\mathrm{Bn}), 4.82(\mathrm{~d}, 1 \mathrm{H}, J=10.8 \mathrm{~Hz}, \mathrm{CH} H \mathrm{Bn}$ ), 4.40 (hept, $1 \mathrm{H}, J=5.9 \mathrm{~Hz}$, CH HFIP), 4.26 (dd, 1H, $J=10.3,4.9 \mathrm{~Hz}, \mathrm{H}-6), 4.10(\mathrm{dd}, 1 \mathrm{H}, J=$ 10.0, $9.1 \mathrm{~Hz}, \mathrm{H}-3$ ), 3.98 (td, $1 \mathrm{H}, J=10.0,4.9 \mathrm{~Hz}, \mathrm{H}-5$ ), 3.80-3.73 (m, $2 \mathrm{H}, \mathrm{H}-4, \mathrm{H}-6), 3.51$ (dd, $1 \mathrm{H}, J=10.1,3.9 \mathrm{~Hz}, \mathrm{H}-2) .{ }^{13} \mathrm{C}-\mathrm{APT}$ NMR $\left(\mathrm{CDCl}_{3}, 101 \mathrm{MHz}, \mathrm{HSQC}\right): \delta 137.6,136.9\left(\mathrm{C}_{\mathrm{q}}\right), 129.3,128.6,128.5$, 128.4, 128.2, $126.1\left(\mathrm{CH}_{\text {arom }}\right), 120.9\left(\mathrm{q}, J=283 \mathrm{~Hz}, \mathrm{CF}_{3}\right), 101.6$ (CHPh), 101.0 (C-1), 82.1 (C-4), 75.9 (C-3), $75.5\left(\mathrm{CH}_{2} \mathrm{Bn}\right), 74.0$, 73.7 (hept, $J=32.8 \mathrm{~Hz}$, CH HFIP), 68.3 (C-6), 64.2 (C-5), 62.5 (C2). HRMS: $[\mathrm{M}+\mathrm{H}]^{+}$calcd for $\mathrm{C}_{23} \mathrm{H}_{21} \mathrm{~F}_{6} \mathrm{~N}_{3} \mathrm{O}_{5}$ 534.14582, found 534.14569 .

Ethyl 2-Azido-3-O-benzoyl-4,6-O-benzylidene-2-deoxy- $\beta$-D-glucopyranoside $(4 A)$. Donor 4 and ethanol were condensed using the general procedure for $\mathrm{Tf}_{2} \mathrm{O} / \mathrm{Ph}_{2} \mathrm{SO}$-mediated glycosylations and purified by flash column chromatography (1:1:0 to $0: 1: 0$ to $0: 19: 1$ pentane/toluene/EtOAc) to yield glycosylation product $4 \mathrm{~A}(36 \mathrm{mg}$, $85 \mu \mathrm{mol}, 85 \%, \alpha / \beta<1: 20)$ as a white solid. $R_{\mathrm{f}} 0.44$ (19:1 toluene/ EtOAc). $[\alpha]_{\mathrm{D}}^{23}=-53.7^{\circ}(c=1.04, \mathrm{DCM})$. IR (thin film): 709, 1001, $1026,1070,1094,1180,1263,1375,1726,2110,2872$. Data for the $\beta$ - anomer: ${ }^{1} \mathrm{H}$ NMR $\left(\mathrm{CDCl}_{3}, 400 \mathrm{MHz}, \mathrm{H}-\mathrm{H}\right.$ COSY, HSQC $): \delta 8.11-$ $8.05\left(\mathrm{~m}, 2 \mathrm{H}, \mathrm{CH}_{\text {arom }}\right), 7.60-7.55\left(\mathrm{~m}, 1 \mathrm{H}, \mathrm{CH}_{\text {arom }}\right), 7.48-7.43(\mathrm{~m}, 2 \mathrm{H}$, $\left.\mathrm{CH}_{\text {arom }}\right), 7.40-7.35\left(\mathrm{~m}, 2 \mathrm{H}, \mathrm{CH}_{\text {arom }}\right), 7.32-7.27$ (m, $\left.3 \mathrm{H}, \mathrm{CH}_{\text {arom }}\right)$, $5.50(\mathrm{~s}, 1 \mathrm{H}, \mathrm{CHPh}), 5.40(\mathrm{t}, 1 \mathrm{H}, J=9.8 \mathrm{~Hz}, \mathrm{H}-3), 4.59(\mathrm{~d}, 1 \mathrm{H}, J=7.9$ $\mathrm{Hz}, \mathrm{H}-1), 4.38$ (dd, $1 \mathrm{H}, J=10.6,5.0 \mathrm{~Hz}, \mathrm{H}-6), 4.02$ (dq, $1 \mathrm{H}, J=9.5$, $7.1 \mathrm{~Hz}, \mathrm{CHH} \mathrm{Et}), 3.83(\mathrm{t}, 1 \mathrm{H}, J=10.3 \mathrm{~Hz}, \mathrm{H}-6), 3.80-3.67(\mathrm{~m}, 2 \mathrm{H}$, $\mathrm{H}-4, \mathrm{CHH} \mathrm{Et}), 3.64$ (dd, $1 \mathrm{H}, J=10.0,7.9 \mathrm{~Hz}, \mathrm{H}-2), 3.56(\mathrm{td}, 1 \mathrm{H}, J=$ 9.7, $5.0 \mathrm{~Hz}, \mathrm{H}-5), 1.32\left(\mathrm{t}, 3 \mathrm{H}, J=7.1 \mathrm{~Hz}, \mathrm{CH}_{3} \mathrm{Et}\right) .{ }^{13} \mathrm{C}-\mathrm{APT}$ NMR $\left(\mathrm{CDCl}_{3}, 101 \mathrm{MHz}, \mathrm{HSQC}\right): \delta 165.5(\mathrm{C}=\mathrm{O} \mathrm{Bz}), 136.9\left(\mathrm{C}_{\mathrm{q}}\right), 133.4$, $130.1\left(\mathrm{CH}_{\text {arom }}\right), 129.7\left(\mathrm{C}_{\mathrm{q}}\right), 129.2,128.6,128.3,126.2\left(\mathrm{CH}_{\text {arom }}\right), 102.8$ (C-1), 101.6 (CHPh), 79.0 (C-4), 71.8 (C-3), 68.7 (C-6), 66.6, 66.6 $\left(\mathrm{C}-5, \mathrm{CH}_{2} \mathrm{Et}\right), 65.0(\mathrm{C}-2), 15.2\left(\mathrm{CH}_{3} \mathrm{Et}\right)$. Diagnostic peaks for the $\alpha$ anomer: ${ }^{1} \mathrm{H}$ NMR ( $\mathrm{CDCl}_{3}, 400 \mathrm{MHz}, \mathrm{H}-\mathrm{H}$ COSY, HSQC): $\delta 5.88$ $(\mathrm{t}, 0.03 \mathrm{H}, J=9.9 \mathrm{~Hz}, \mathrm{H}-3), 5.53(\mathrm{~s}, 0.03 \mathrm{H}, \mathrm{CHPh}), 5.06(\mathrm{~d}, 0.03 \mathrm{H}, J=$ $3.6 \mathrm{~Hz}, \mathrm{H}-1), 4.32(\mathrm{dd}, 0.03 \mathrm{H}, J=10.4,4.9 \mathrm{~Hz}, \mathrm{H}-6), 3.32(\mathrm{dd}, 0.03 \mathrm{H}$, $J=10.3,3.6 \mathrm{~Hz}, \mathrm{H}-2)$. HRMS: $\left[\mathrm{M}+\mathrm{NH}_{4}\right]^{+}$calcd for $\mathrm{C}_{22} \mathrm{H}_{27} \mathrm{~N}_{4} \mathrm{O}_{6}$ 443.19251, found 443.19234.

Cyclohexyl 2-Azido-3-O-benozyl-4,6-O-benzylidene-2-deoxy- $\beta$-Dglucopyranoside (4B). Donor 4 and cyclohexanol were condensed using the general procedure for $\mathrm{Tf}_{2} \mathrm{O} / \mathrm{Ph}_{2} \mathrm{SO}$-mediated glycosylations and purified by flash column chromatography (1:1:0 to $0: 1: 0$ to $0: 19: 1$ pentane/toluene/EtOAc) to yield glycosylation product 4B $(43.6 \mathrm{mg}$, $91 \mu \mathrm{mol}, 91 \%, \alpha / \beta<1: 20)$ as a white solid. $R_{\mathrm{f}} 0.18$ (toluene). $[\alpha]_{\mathrm{D}}^{23}=$ $-41.2^{\circ}(c=0.87, \mathrm{DCM})$. IR (thin film): 613, 708, 999, 1096, 1263, 1730, 2110, 2859, 2934. ${ }^{1} \mathrm{H}$ NMR ( $\mathrm{CDCl}_{3}, 400 \mathrm{MHz}, \mathrm{H}-\mathrm{H}$ COSY, HSQC): $\delta 8.11-8.05\left(\mathrm{~m}, 2 \mathrm{H}, \mathrm{CH}_{\text {arom }}\right), 7.61-7.54\left(\mathrm{~m}, 1 \mathrm{H}, \mathrm{CH}_{\text {arom }}\right)$, 7.49-7.42 (m, $\left.2 \mathrm{H}, \mathrm{CH}_{\text {arom }}\right), 7.41-7.35\left(\mathrm{~m}, 2 \mathrm{H}, \mathrm{CH}_{\text {arom }}\right), 7.31-7.27$ $\left(\mathrm{m}, 3 \mathrm{H}, \mathrm{CH}_{\text {arom }}\right), 5.50(\mathrm{~s}, 1 \mathrm{H}, \mathrm{CHPh}), 5.38(\mathrm{t}, 1 \mathrm{H}, J=9.9 \mathrm{~Hz}, \mathrm{H}-3)$, $4.70(\mathrm{~d}, 1 \mathrm{H}, J=7.9 \mathrm{~Hz}, \mathrm{H}-1), 4.37$ (dd, $1 \mathrm{H}, J=10.6,5.0 \mathrm{~Hz}, \mathrm{H}-6)$, 3.89-3.71 (m, 3H, H-4, H-6, CHO Cyc), 3.64 (dd, $1 \mathrm{H}, J=10.1,7.9$ $\mathrm{Hz}, \mathrm{H}-2), 3.55$ (td, $1 \mathrm{H}, \mathrm{J}=9.8,5.0 \mathrm{~Hz}, \mathrm{H}-5), 2.04-1.90(\mathrm{~m}, 2 \mathrm{H}, 2 \times$ $\mathrm{CHH} \mathrm{Cyc),} 1.85-1.73(\mathrm{~m}, 2 \mathrm{H}, 2 \times \mathrm{CHH} \mathrm{Cyc}), 1.58-1.40(\mathrm{~m}, 3 \mathrm{H}$, $\mathrm{CHH}$ Cyc, $2 \times \mathrm{CHH}$ Cyc), $1.39-1.20(\mathrm{~m}, 3 \mathrm{H}, 3 \times \mathrm{CHH} \mathrm{Cyc}) .{ }^{13} \mathrm{C}-$ APT NMR ( $\left.\mathrm{CDCl}_{3}, 101 \mathrm{MHz}, \mathrm{HSQC}\right): \delta 165.5$ (C= O Bz), 136.9 $\left(\mathrm{C}_{\mathrm{q}}\right), 133.4,130.0\left(\mathrm{CH}_{\text {arom }}\right), 129.7\left(\mathrm{C}_{\mathrm{q}}\right), 129.2,128.5,128.3,126.2$ $\left(\mathrm{CH}_{\text {arom }}\right), 101.6(\mathrm{CHPh}), 101.2(\mathrm{C}-1), 79.0(\mathrm{C}-4), 78.8(\mathrm{CH} \mathrm{Cyc})$, 71.8 (C-3), 68.7 (C-6), 66.6 (C-5), 65.2 (C-2), 33.7, 31.7, 25.6, 24.1, $23.9\left(\mathrm{CH}_{2}\right.$ Cyc). HRMS: $\left[\mathrm{M}+\mathrm{NH}_{4}\right]^{+}$calcd for $\mathrm{C}_{26} \mathrm{H}_{33} \mathrm{~N}_{4} \mathrm{O}_{6}$ 497.23946, found 497.23932.

Methyl 6-O-(2-Azido-3-O-benzoyl-4,6-O-benzylidene-2-deoxy- $\alpha$ ) $\beta$-D-glucopyranosyl)-2,3,4-tri-O-benzyl- $\alpha$-D-glucopyranoside (4C). Donor 4 and acceptor 25 were condensed using the general procedure for $\mathrm{Tf}_{2} \mathrm{O} / \mathrm{Ph}_{2} \mathrm{SO}$-mediated glycosylations (for an additional $18 \mathrm{~h}$ at $-40{ }^{\circ} \mathrm{C}$ ) and purified by flash column chromatography (19:1 to $3 / 1$ pentane/EtOAc) to yield glycosylation product $4 \mathrm{C}(67 \mathrm{mg}, 79 \mu \mathrm{mol}$, $79 \%, \alpha / \beta=1: 14)$ as a white solid. $R_{\mathrm{f}} 0.24\left(4: 1\right.$ pentane/EtOAc). $[\alpha]_{\mathrm{D}}^{20}$ $=-17.5^{\circ}\left(c=1.34, \mathrm{CHCl}_{3}\right)$. IR (thin film): 696, 748, 1002, 1028, $1068,1092,1263,1313,1369,1452,1730,2110,2872,2918$. Data for the $\beta$-anomer: ${ }^{1} \mathrm{H}$ NMR $\left(\mathrm{CDCl}_{3}, 400 \mathrm{MHz}, \mathrm{H}-\mathrm{H}\right.$ COSY, HSQC, HMBC): $\delta 8.09-8.04\left(\mathrm{~m}, 2 \mathrm{H}, \mathrm{CH}_{\text {arom }}\right), 7.60-7.53\left(\mathrm{~m}, 1 \mathrm{H}, \mathrm{CH}_{\text {arom }}\right)$, 7.48-7.41 (m, 2H, $\left.\mathrm{CH}_{\text {arom }}\right), 7.40-7.24\left(\mathrm{~m}, 20 \mathrm{H}, \mathrm{CH}_{\text {arom }}\right), 5.47(\mathrm{~s}, 1 \mathrm{H}$, $\mathrm{CHPh}), 5.42\left(\mathrm{t}, 1 \mathrm{H}, J=9.8 \mathrm{~Hz}, \mathrm{H}-3^{\prime}\right), 5.00(\mathrm{~d}, 1 \mathrm{H}, J=10.9 \mathrm{~Hz}, \mathrm{CHH}$ $\mathrm{Bn}), 4.95(\mathrm{~d}, 1 \mathrm{H}, J=11.1 \mathrm{~Hz}, \mathrm{CHH} \mathrm{Bn}), 4.84(\mathrm{~d}, 1 \mathrm{H}, J=11.0 \mathrm{~Hz}$, CHH Bn), 4.80 (d, 1H, J = 12.2 Hz, CHH Bn), 4.68-4.63 (m, 2H, 2 $\times \mathrm{CHH} \mathrm{Bn}), 4.61(\mathrm{~d}, 1 \mathrm{H}, J=3.5 \mathrm{~Hz}, \mathrm{H}-1), 4.43(\mathrm{~d}, 1 \mathrm{H}, J=8.0 \mathrm{~Hz}, \mathrm{H}-$ $\left.1^{\prime}\right), 4.33\left(\mathrm{dd}, 1 \mathrm{H}, J=10.5,5.0 \mathrm{~Hz}, \mathrm{H}-6^{\prime}\right), 4.12(\mathrm{~d}, 1 \mathrm{H}, J=9.1 \mathrm{~Hz}, \mathrm{H}-$ 6), $4.02(\mathrm{t}, 1 \mathrm{H}, J=9.3 \mathrm{~Hz}, \mathrm{H}-3), 3.84-3.72\left(\mathrm{~m}, 4 \mathrm{H}, \mathrm{H}-4^{\prime}, \mathrm{H}-5, \mathrm{H}-6\right.$, H-6'), 3.69 (dd, $\left.1 \mathrm{H}, J=9.9,8.0 \mathrm{~Hz}, \mathrm{H}-2^{\prime}\right), 3.57(\mathrm{t}, 1 \mathrm{H}, J=9.2 \mathrm{~Hz}, \mathrm{H}-$ 4), $3.54(\mathrm{dd}, 1 \mathrm{H}, J=9.7,3.6 \mathrm{~Hz}, \mathrm{H}-2), 3.49(\mathrm{td}, 1 \mathrm{H}, J=9.8,5.0 \mathrm{~Hz}, \mathrm{H}-$ $\left.5^{\prime}\right), 3.39$ (s, 3H, $\left.\mathrm{CH}_{3} \mathrm{OMe}\right) .{ }^{13} \mathrm{C}-\mathrm{APT} \mathrm{NMR}\left(\mathrm{CDCl}_{3}, 101 \mathrm{MHz}\right.$, HSQC, HMBC): $\delta 165.4(\mathrm{C}=\mathrm{O}), 138.7,138.3,138.2,136.7$ $\left(\mathrm{CH}_{\text {arom }}\right), 133.5,130.0\left(\mathrm{CH}_{\text {arom }}\right), 129.4\left(\mathrm{C}_{\mathrm{q}} \mathrm{Bz}\right), 129.1,128.6,128.6$, $128.5,128.5,128.3,128.3,128.1,128.0,128.0,127.9,127.9,127.7$, $126.1\left(\mathrm{CH}_{\text {arom }}\right), 102.6\left(\mathrm{C}-1^{\prime}\right), 101.5(\mathrm{CHPh}), 98.3(\mathrm{C}-1), 82.1(\mathrm{C}-3)$, 79.8 (C-2), 78.7 (C-4'), 77.7 (C-4), 75.8, 75.0, $73.6\left(\mathrm{CH}_{2} \mathrm{Bn}\right), 71.9$ $\left(\mathrm{C}-3^{\prime}\right), 69.7$ (C-5), 68.9 (C-6), $68.5\left(\mathrm{C}-6^{\prime}\right), 66.6\left(\mathrm{C}-5^{\prime}\right), 65.2\left(\mathrm{C}-2^{\prime}\right)$, $55.4\left(\mathrm{CH}_{3} \mathrm{OMe}\right)$. Diagnostic peaks for the $\alpha$-anomer: ${ }^{1} \mathrm{H}$ NMR $\left(\mathrm{CDCl}_{3}, 400 \mathrm{MHz}\right): \delta 5.79\left(\mathrm{t}, 0.07 \mathrm{H}, J=9.9 \mathrm{~Hz}, \mathrm{H}-3^{\prime}\right), 5.50(\mathrm{~s}$, $0.07 \mathrm{H}, \mathrm{CHPh}), 5.08\left(\mathrm{~d}, 0.07 \mathrm{H}, J=3.5 \mathrm{~Hz}, \mathrm{H}-1^{\prime}\right), 4.24(\mathrm{dd}, 0.07 \mathrm{H}, J=$ 10.3, $\left.4.8 \mathrm{~Hz}, \mathrm{H}-6^{\prime}\right), 3.41$ (s, $\left.0.21 \mathrm{H}, \mathrm{CH}_{3} \mathrm{OMe}\right) .{ }^{13} \mathrm{C}-\mathrm{APT}$ NMR 
$\left(\mathrm{CDCl}_{3}, 101 \mathrm{MHz}\right): \delta 101.7(\mathrm{CHPh}), 99.3\left(\mathrm{C}-1^{\prime}\right), 98.1$ (C-1), 82.1, 80.0, 79.7, 77.6, 75.8, 75.2, 70.0, 69.4, 67.0, 62.8, 62.1, 55.4. HRMS: $\left[\mathrm{M}+\mathrm{NH}_{4}\right]^{+}$calcd for $\mathrm{C}_{48} \mathrm{H}_{53} \mathrm{~N}_{4} \mathrm{O}_{11} 861.37053$, found 861.37064.

2-Fluoroethyl 2-Azido-3-O-benzoyl-4,6-O-benzylidene-2-deoxy$\alpha / \beta$-D-glucopyranoside (4D). Donor 4 and 2 -fluoroethanol were condensed using the general procedure for $\mathrm{Tf}_{2} \mathrm{O} / \mathrm{Ph}_{2} \mathrm{SO}$-mediated glycosylations and purified by flash column chromatography (1:1:0 to $0: 1: 0$ to $0: 16: 1$ pentane/toluene/EtOAc) to yield glycosylation product 4D (36.6 $\mathrm{mg}, 83 \mu \mathrm{mol}, 83 \%, \alpha / \beta=1: 6.5)$ as a white solid. $R_{\mathrm{f}} 0.41$ (19:1 toluene/EtOAc). IR (thin film): 700, 748, 879, 1001, $1026,1070,1093,1179,1261,1722,2108,2868$. Data for the $\beta$ anomer: ${ }^{1} \mathrm{H}$ NMR $\left(\mathrm{CDCl}_{3}, 400 \mathrm{MHz}, \mathrm{H}-\mathrm{H}\right.$ COSY, HSQC $): \delta 8.10-$ $8.05\left(\mathrm{~m}, 2 \mathrm{H}, \mathrm{CH}_{\text {arom }}\right), 7.61-7.54\left(\mathrm{~m}, 1 \mathrm{H}, \mathrm{CH}_{\text {arom }}\right), 7.49-7.43(\mathrm{~m}, 2 \mathrm{H}$, $\mathrm{CH}_{\text {arom }}$ ), 7.41-7.36 (m, $\left.2 \mathrm{H}, \mathrm{CH}_{\text {arom }}\right), 7.33-7.27$ (m, 3H, $\left.\mathrm{CH}_{\text {arom }}\right)$, $5.50(\mathrm{~s}, 1 \mathrm{H}, \mathrm{CHPh}), 5.41(\mathrm{t}, 1 \mathrm{H}, J=9.8 \mathrm{~Hz}, \mathrm{H}-3), 4.69(\mathrm{ddt}, 1 \mathrm{H}, J=$ 4.6, 3.2, $\left.1.8 \mathrm{~Hz}, \mathrm{CHH}-\mathrm{CH}_{2} \mathrm{~F}\right), 4.65(\mathrm{~d}, 1 \mathrm{H}, J=7.9 \mathrm{~Hz}, \mathrm{H}-1), 4.58$ (ddt, $\left.1 \mathrm{H}, J=4.5,3.3,1.8 \mathrm{~Hz}, \mathrm{CHH}-\mathrm{CH}_{2} \mathrm{~F}\right), 4.38(\mathrm{dd}, 1 \mathrm{H}, J=10.5$, $4.9 \mathrm{~Hz}, \mathrm{H}-6$ ), 4.14 (dddd, $1 \mathrm{H}, J=30.3,11.9,4.6,3.1 \mathrm{~Hz}, \mathrm{CHHF}$ ), 3.95 (dddd, $1 \mathrm{H}, J=27.1,12.1,5.5,3.4 \mathrm{~Hz}, \mathrm{CHHF}), 3.83(\mathrm{t}, 1 \mathrm{H}, J=10.3$ $\mathrm{Hz}, \mathrm{H}-6), 3.79$ (t, $1 \mathrm{H}, J=9.5 \mathrm{~Hz}, \mathrm{H}-4), 3.69$ (dd, $1 \mathrm{H}, J=10.0,7.9 \mathrm{~Hz}$, $\mathrm{H}-2), 3.57(\mathrm{td}, 1 \mathrm{H}, J=9.7,5.0 \mathrm{~Hz}, \mathrm{H}-5) .{ }^{13} \mathrm{C}-\mathrm{APT}$ NMR $\left(\mathrm{CDCl}_{3}, 101\right.$ $\mathrm{MHz}, \mathrm{HSQC}): \delta 165.4(\mathrm{C}=\mathrm{O}), 136.8\left(\mathrm{C}_{\mathrm{q}} \mathrm{Ph}\right), 133.5,130.0$ $\left(\mathrm{CH}_{\text {arom }}\right), 129.5\left(\mathrm{C}_{\mathrm{q}} \mathrm{Bz}\right), 129.2,128.6,128.5,128.3,126.3,126.2$ $\left(\mathrm{CH}_{\text {arom }}\right), 103.0(\mathrm{C}-1), 101.6(\mathrm{CHPh}), 82.5\left(\mathrm{~d}, J=170.5 \mathrm{~Hz}, \mathrm{CH}_{2} \mathrm{~F}\right)$, 78.8 (C-4), 71.7 (C-3), 69.5 (d, $\left.\mathrm{J}=20.2 \mathrm{~Hz}, \mathrm{CH}_{2}-\mathrm{CH}_{2} \mathrm{~F}\right), 68.5$ (C6), 66.7 (C-5), 64.9 (C-2). Diagnostic peaks for the $\alpha$-anomer: ${ }^{1} \mathrm{H}$ NMR $\left(\mathrm{CDCl}_{3}, 400 \mathrm{MHz}\right): \delta 5.89(\mathrm{t}, 0.17 \mathrm{H}, J=9.9 \mathrm{~Hz}, \mathrm{H}-3), 5.53(\mathrm{~s}$, $0.17 \mathrm{H}, \mathrm{CHPh}), 5.12(\mathrm{~d}, 0.17 \mathrm{H}, J=3.6 \mathrm{~Hz}, \mathrm{H}-1), 4.33(\mathrm{dd}, 0.17 \mathrm{H}, J=$ 10.4, $5.0 \mathrm{~Hz}, \mathrm{H}-6), 3.38$ (dd, $0.17 \mathrm{H}, J=10.4,3.6 \mathrm{~Hz}, \mathrm{H}-2) .{ }^{13} \mathrm{C}-\mathrm{APT}$ NMR $\left(\mathrm{CDCl}_{3}, 101 \mathrm{MHz}\right): \delta 101.8,99.5(\mathrm{C}-1), 82.4(\mathrm{~d}, J=170.8 \mathrm{~Hz})$, 79.6, 68.9, $67.8(\mathrm{~d}, J=20.2 \mathrm{~Hz}), 63.1,61.8$. HRMS: $\left[\mathrm{M}+\mathrm{NH}_{4}\right]^{+}$calcd for $\mathrm{C}_{22} \mathrm{H}_{26} \mathrm{FN}_{4} \mathrm{O}_{6} 461.18309$, found 461.18292.

Methyl 4-O-(2-Azido-3-O-benzoyl-4,6-O-benzylidene-2-deoxy- $\alpha$ ) $\beta$-D-glucopyranosyl)-2,3,6-tri-O-benzyl- $\alpha$-D-glucopyranoside (4E). Donor 4 and acceptor 26 were condensed using the general procedure for $\mathrm{Tf}_{2} \mathrm{O} / \mathrm{Ph}_{2} \mathrm{SO}$-mediated glycosylations (for an additional $18 \mathrm{~h}$ at $-40{ }^{\circ} \mathrm{C}$ ) and purified by flash column chromatography (19:1 to $4: 1$ pentane/EtOAc) to yield glycosylation product $4 \mathrm{E}(60 \mathrm{mg}, 71 \mu \mathrm{mol}$, $71 \%, \alpha / \beta=1: 6)$ as a white solid. $R_{\mathrm{f}} 0.67$ (4:1 pentane/EtOAc). IR (thin film): 696, 733, 914, 999, 1026, 1045, 1090, 1177, 1263, 1314, $1366,1452,1730,2108,2866,2899$. Data for the $\beta$-anomer: ${ }^{1} \mathrm{H}$ NMR $\left(\mathrm{CDCl}_{3}, 400 \mathrm{MHz}, \mathrm{H}-\mathrm{H}\right.$ COSY, HSQC, HMBC): $\delta 8.10-8.04(\mathrm{~m}$, $\left.2 \mathrm{H}, \mathrm{CH}_{\text {arom }}\right), 7.61-7.53\left(\mathrm{~m}, 1 \mathrm{H}, \mathrm{CH}_{\text {arom }}\right), 7.49-7.24(\mathrm{~m}, 22 \mathrm{H}$, $\left.\mathrm{CH}_{\text {arom }}\right), 5.40(\mathrm{~s}, 1 \mathrm{H}, \mathrm{CHPh}), 5.19\left(\mathrm{t}, 1 \mathrm{H}, J=9.8 \mathrm{~Hz}, \mathrm{H}-3^{\prime}\right), 4.90(\mathrm{~d}$, $1 \mathrm{H}, J=10.8 \mathrm{~Hz}, \mathrm{CHH} \mathrm{Bn}), 4.83$ (d, $1 \mathrm{H}, J=10.8 \mathrm{~Hz}, \mathrm{CHH} \mathrm{Bn}), 4.81-$ $4.73(\mathrm{~m}, 2 \mathrm{H}, 2 \times \mathrm{CHH} \mathrm{Bn}), 4.67-4.60(\mathrm{~m}, 2 \mathrm{H}, \mathrm{CHH} \mathrm{Bn}, \mathrm{H}-1), 4.42$ (d, $1 \mathrm{H}, J=12.0 \mathrm{~Hz}, \mathrm{CHH} \mathrm{Bn}), 4.31\left(\mathrm{~d}, 1 \mathrm{H}, J=8.0 \mathrm{~Hz}, \mathrm{H}-1^{\prime}\right), 4.17$ $\left(\mathrm{dd}, 1 \mathrm{H}, J=10.6,5.0 \mathrm{~Hz}, \mathrm{H}-6^{\prime}\right), 4.08-3.98(\mathrm{t}, 1 \mathrm{H}, J=9.4 \mathrm{~Hz}, \mathrm{H}-4)$, $3.96(\mathrm{dd}, 1 \mathrm{H}, J=10.8,2.4 \mathrm{~Hz}, \mathrm{H}-6), 3.86(\mathrm{t}, 1 \mathrm{H}, J=9.3 \mathrm{~Hz}, \mathrm{H}-3)$, 3.79-3.74 (m, 1H, H-5), $3.71(\mathrm{dd}, 1 \mathrm{H}, J=10.7,1.7 \mathrm{~Hz}, \mathrm{H}-6), 3.61(\mathrm{t}$, $\left.1 \mathrm{H}, J=9.5 \mathrm{~Hz}, \mathrm{H}-4^{\prime}\right), 3.54$ (dd, $\left.1 \mathrm{H}, J=9.6,3.7 \mathrm{~Hz}, \mathrm{H}-2\right), 3.52-3.45$ (m, 2H, H-2', H-6'), 3.39 (s, 3H, $\mathrm{CH}_{3} \mathrm{OMe}$ ), $3.08(\mathrm{td}, 1 \mathrm{H}, J=9.7,5.0$ $\left.\mathrm{Hz}, \mathrm{H}-5^{\prime}\right) .{ }^{13} \mathrm{C}-\mathrm{APT}$ NMR $\left(\mathrm{CDCl}_{3}, 101 \mathrm{MHz}, \mathrm{HSQC}, \mathrm{HMBC}\right): \delta$ $165.3(\mathrm{C}=\mathrm{O}), 139.3,138.3,137.6,136.9\left(\mathrm{C}_{\mathrm{q}}\right), 133.4,129.9\left(\mathrm{CH}_{\text {arom }}\right)$, $128.9\left(\mathrm{C}_{\mathrm{q}} \mathrm{Bz}\right), 128.6,128.5,128.5,128.3,128.3,128.2,127.9,127.8$, $126.2\left(\mathrm{CH}_{\text {arom }}\right), 101.5,101.4\left(\mathrm{CHPh}, \mathrm{C}-1^{\prime}\right), 98.4(\mathrm{C}-1), 80.1(\mathrm{C}-3)$, 79.1 (C-2), 78.9 (C-4'), 76.8 (C-4), 75.6, 73.7, $73.6\left(\mathrm{CH}_{2} \mathrm{Bn}\right), 72.0$ (C-3'), 69.7 (C-5), $68.6\left(\mathrm{C}-6^{\prime}\right), 67.8$ (C-6), $66.1\left(\mathrm{C}-5^{\prime}\right), 65.6\left(\mathrm{C}-2^{\prime}\right)$, 55.5 (OMe). Diagnostic peaks for the $\alpha$-anomer: ${ }^{1} \mathrm{H}$ NMR $\left(\mathrm{CDCl}_{3}\right.$, $400 \mathrm{MHz}): \delta 5.91\left(\mathrm{~d}, 0.17 \mathrm{H}, J=3.9 \mathrm{~Hz}, \mathrm{H}-1^{\prime}\right), 5.80(\mathrm{t}, 0.17 \mathrm{H}, J=10.0$ $\left.\mathrm{Hz}, \mathrm{H}-3^{\prime}\right), 5.47$ (s, 0.17H, CHPh), $5.15(\mathrm{~d}, 0.17 \mathrm{H}, J=10.7 \mathrm{~Hz}, \mathrm{CHH}$ $\mathrm{Bn}), 4.74(\mathrm{~d}, 0.17 \mathrm{H}, J=12.0 \mathrm{~Hz}, \mathrm{CHH} \mathrm{Bn}), 4.09(\mathrm{t}, 0.17 \mathrm{H}, J=9.2$ $\mathrm{Hz}), 3.29\left(\mathrm{dd}, 0.17 \mathrm{H}, J=10.5,3.9 \mathrm{~Hz}, \mathrm{H}-2^{\prime}\right) .{ }^{13} \mathrm{C}-\mathrm{APT} \mathrm{NMR}\left(\mathrm{CDCl}_{3}\right.$, $101 \mathrm{MHz}): \delta 165.6,138.6,138.1,137.9,137.0,133.4,130.0,129.6$, $129.0,128.6,128.6,128.5,128.4,128.2,128.1,127.6,127.6,127.4$, 101.6 (CHPh), 98.6 (C-1'), 97.7 (C-1), 82.1, 80.7, 79.5, 75.0, 73.6, 73.3, 72.8, 69.5, 69.3, 68.9, 68.7, 63.7, 61.9, 55.5. HRMS: $\left[\mathrm{M}+\mathrm{NH}_{4}\right]^{+}$ calcd for $\mathrm{C}_{48} \mathrm{H}_{53} \mathrm{~N}_{4} \mathrm{O}_{11} 861.37053$, found 861.37081.

Methyl (Methyl 4-O-[2-Azido-3-O-benzoyl-4,6-O-benzylidene-2deoxy- $\alpha / \beta$-D-glucopyranosyl]-2,3-di-O-benzyl- $\alpha$-D-glucopyranosyl uronate) (4F). Donor 4 and acceptor 27 were condensed using the general procedure for $\mathrm{Tf}_{2} \mathrm{O} / \mathrm{Ph}_{2} \mathrm{SO}$-mediated glycosylations (for an additional $18 \mathrm{~h}$ at $-40{ }^{\circ} \mathrm{C}$ ) and purified by flash column chromatography (19:1 to $4: 1$ pentane/EtOAc) to yield glycosylation product $4 \mathrm{~F}$ (46 mg, $59 \mu \mathrm{mol}, 59 \%, \alpha / \beta=1: 1.4$ ) as a white solid. $R_{\mathrm{f}}$ 0.56 (4:1 pentane/EtOAc). IR (thin film): 698, 750, 991, 1026, 1047, $1092,1178,1263,1452,1730,2110,2868,2938$. Data reported for a 0.7:1 mixture of anomers: ${ }^{1} \mathrm{H}$ NMR $\left(\mathrm{CDCl}_{3}, 400 \mathrm{MHz}, \mathrm{H}-\mathrm{H}\right.$ COSY, HSQC, HMBC): $\delta 8.09-8.03\left(\mathrm{~m}, 3.4 \mathrm{H}, \mathrm{CH}_{\text {arom }}\right), 7.61-7.52(\mathrm{~m}$, $\left.1.7 \mathrm{H}, \mathrm{CH}_{\text {arom }}\right), 7.49-7.25\left(\mathrm{~m}, 28.9 \mathrm{H}, \mathrm{CH}_{\text {arom }}\right), 5.76(\mathrm{dd}, 0.7 \mathrm{H}, J=9.5$, $\left.10.3 \mathrm{~Hz}, \mathrm{H}-3^{\prime}{ }_{\alpha}\right), 5.70\left(\mathrm{~d}, 0.7 \mathrm{H}, J=3.9 \mathrm{~Hz}, \mathrm{H}^{-1}{ }_{\alpha}{ }_{\alpha}\right), 5.47(\mathrm{~s}, 0.7 \mathrm{H}$, $\left.\mathrm{CHPh}_{\alpha}\right), 5.41\left(\mathrm{~s}, 1 \mathrm{H}, \mathrm{CHPh}{ }_{\beta}\right), 5.36\left(\mathrm{t}, 1 \mathrm{H}, J=9.8 \mathrm{~Hz}, \mathrm{H}-3^{\prime}{ }_{\beta}\right), 5.10(\mathrm{~d}$, $\left.0.7 \mathrm{H}, J=10.6 \mathrm{~Hz}, \mathrm{CHH} \mathrm{Bn}{ }_{\alpha}\right), 4.93\left(\mathrm{~d}, 1 \mathrm{H}, J=10.9 \mathrm{~Hz}, \mathrm{CHH} \mathrm{Bn}{ }_{\beta}\right.$ ), $\left.4.87(\mathrm{~d}, 1 \mathrm{H}, J=10.9 \mathrm{~Hz}, \mathrm{CHH} \mathrm{Bn})_{\beta}\right), 4.83(\mathrm{~d}, 0.7 \mathrm{H}, J=10.6 \mathrm{~Hz}, \mathrm{CHH}$ $\left.\mathrm{Bn}_{\alpha}\right), 4.79\left(\mathrm{~d}, 1 \mathrm{H}, J=13.6 \mathrm{~Hz}, \mathrm{CHH} \mathrm{Bn}{ }_{\beta}\right), 4.76(\mathrm{~d}, 0.7 \mathrm{H}, J=13.6 \mathrm{~Hz}$, $\left.\mathrm{CHH} \mathrm{Bn}{ }_{\alpha}\right), 4.66-4.62\left(\mathrm{~m}, 2 \mathrm{H}, \mathrm{CHH} \mathrm{Bn}_{\beta}, \mathrm{H}^{\prime}{ }^{\prime}{ }_{\beta}\right), 4.61-4.58(\mathrm{~m}$, $\left.2.4 \mathrm{H}, \mathrm{CHH} \mathrm{Bn}{ }_{\alpha}, \mathrm{H}-1_{\alpha}, \mathrm{H}-1_{\beta}\right), 4.31\left(\mathrm{dd}, 0.7 \mathrm{H}, J=10.0,4.6 \mathrm{~Hz}, \mathrm{H}-6^{\prime}{ }_{\alpha}\right)$, 4.28 (d, $\left.0.7 \mathrm{H}, J=9.7 \mathrm{~Hz}, \mathrm{H}-5_{\alpha}\right), 4.23$ (d, $\left.1 \mathrm{H}, J=9.9 \mathrm{~Hz}, \mathrm{H}-5_{\beta}\right), 4.19-$ 4.06 (m, 3.4H, H- $\left.{ }_{\alpha}, \mathrm{H}-4_{\alpha}, \mathrm{H}-4_{\beta}, \mathrm{H}-6^{\prime}{ }_{\beta}\right), 3.91(\mathrm{t}, 1 \mathrm{H}, J=9.2 \mathrm{~Hz}, \mathrm{H}-3)$, 3.85 (s, 2.1H, $\mathrm{CH}_{3} \mathrm{CO}_{2} \mathrm{Me}_{\alpha}$ ), 3.83 (s, $3 \mathrm{H}, \mathrm{CH}_{3} \mathrm{CO}_{2} \mathrm{Me}_{\beta}$ ), 3.79-3.59 $\left(\mathrm{m}, 3.8 \mathrm{H}, \mathrm{H}-2_{\alpha}, \mathrm{H}-4^{\prime}{ }_{\alpha}, \mathrm{H}-4^{\prime}{ }_{\beta}, \mathrm{H}-5^{\prime}{ }_{\alpha}, \mathrm{H}-6^{\prime}{ }_{\alpha}\right), 3.59-3.44\left(\mathrm{~m}, 4 \mathrm{H}, \mathrm{H}-2_{\beta}\right.$, $\left.\mathrm{H}-2^{\prime}{ }_{\beta}, \mathrm{H}-5^{\prime}{ }_{\beta}, \mathrm{H}-6^{\prime}{ }_{\beta}\right), 3.43$ (s, $3 \mathrm{H}, \mathrm{CH}_{3} \mathrm{OMe}_{\beta}$ ), 3.42 (s, $2.1 \mathrm{H}, \mathrm{CH}_{3}$ $\left.\mathrm{OMe}_{\alpha}\right), 3.31\left(\mathrm{dd}, 1 \mathrm{H}, J=10.4,3.9 \mathrm{~Hz}, \mathrm{H}-2^{\prime}{ }_{\alpha}\right) .{ }^{13} \mathrm{C}-\mathrm{APT} \mathrm{NMR}$ $\left(\mathrm{CDCl}_{3}, 101 \mathrm{MHz}, \mathrm{HSQC}, \mathrm{HMBC}\right): \delta 170.2,169.9\left(\mathrm{C}=\mathrm{O} \mathrm{CO}_{2} \mathrm{Me}\right)$, $165.5,165.3(\mathrm{C}=\mathrm{O} \mathrm{Bz}), 139.1,138.4,138.0,137.7,137.0,136.8\left(\mathrm{C}_{\mathrm{q}}\right.$ $\mathrm{Bn}, \mathrm{CHPh}), 133.4,133.4,130.0,130.0\left(\mathrm{CH}_{\text {arom }}\right), 129.5,129.5\left(\mathrm{C}_{\mathrm{q}} \mathrm{Bz}\right)$, $129.1,129.1,128.7,128.6,128.5,128.5,128.5,128.3,128.3,128.2$, $128.1,127.8,127.7,127.6,127.5,126.2,126.2\left(\mathrm{CH}_{\text {arom }}\right), 102.3\left(\mathrm{C}-1^{\prime}{ }_{\beta}\right)$, $101.7\left(\mathrm{CHPh}_{\alpha}\right), 101.5\left(\mathrm{CHPh}_{\beta}\right), 99.0\left({\mathrm{C}-1^{\prime}}_{\alpha}\right), 98.8\left(\mathrm{C}-1_{\beta}\right), 98.5(\mathrm{C}-$ $\left.1_{\alpha}\right), 81.1\left(\mathrm{C}-3_{\alpha}\right), 79.9\left(\mathrm{C}-2_{\alpha}\right), 79.5,79.5\left(\mathrm{C}-3_{\beta}, \mathrm{C}-4_{\beta}\right), 79.2\left(\mathrm{C}-4_{\alpha}^{\prime}\right)$, 78.9, $78.7\left(\mathrm{C}-2_{\beta}, \mathrm{C}-4^{\prime}{ }_{\beta}\right), 75.7,75.4\left(\mathrm{CH}_{2} \mathrm{Bn}\right), 75.2\left(\mathrm{C}-4_{\alpha}\right), 73.9,73.6$ $\left(\mathrm{CH}_{2} \mathrm{Bn}\right), 71.9\left(\mathrm{C}-3^{\prime}{ }_{\beta}\right), 69.8,69.8\left(\mathrm{C}-5_{\alpha}, \mathrm{C}-5_{\beta}\right), 69.6\left(\mathrm{C}-3^{\prime}{ }_{\alpha}\right), 68.5$, $68.4\left(\mathrm{C}-6^{\prime}{ }_{\alpha}, \mathrm{C}-6^{\prime}{ }_{\beta}\right), 66.5\left(\mathrm{C}-5^{\prime}{ }_{\beta}\right), 65.6\left(\mathrm{C}-2^{\prime}{ }_{\beta}\right), 63.2\left(\mathrm{C}-5^{\prime}{ }_{\alpha}\right), 61.7$ (C$\left.2^{\prime}{ }_{\alpha}\right)$, 56.0, 56.0 $\left(\mathrm{CH}_{3} \mathrm{OMe}\right), 53.1,53.0\left(\mathrm{CH}_{3} \mathrm{CO}_{2} \mathrm{Me}\right)$. HRMS: [M + $\left.\mathrm{NH}_{4}\right]^{+}$calcd for $\mathrm{C}_{42} \mathrm{H}_{47} \mathrm{~N}_{4} \mathrm{O}_{12} 799.31850$, found 799.31869 .

2,2-Difluoroethyl 2-Azido-3-O-benzoyl-4,6-O-benzylidene-2deoxy- $\alpha / \beta$-D-glucopyranoside (4G). Donor 4 and 2,2-difluoroethanol were condensed using the general procedure for $\mathrm{Tf}_{2} \mathrm{O} / \mathrm{Ph}_{2} \mathrm{SO}$ mediated glycosylations and purified by flash column chromatography (1:1:0 to $0: 1: 0$ to $0: 19: 1$ pentane/toluene/EtOAc) to yield glycosylation product $4 \mathrm{G}(38.6 \mathrm{mg}, 84 \mu \mathrm{mol}, 84 \%, \alpha / \beta=2.7: 1)$ as a white solid. $R_{\mathrm{f}} 0.49$ (19:1 toluene/EtOAc). IR (thin film): 709, 997, $1026,1069,1094,1265,1726,2108,2870$. Data for the $\alpha$-anomer: ${ }^{1} \mathrm{H}$ NMR ( $\mathrm{CDCl}_{3}, 400 \mathrm{MHz}, \mathrm{H}-\mathrm{H}$ COSY, HSQC): $\delta 8.11-8.05(\mathrm{~m}, 2 \mathrm{H}$, $\left.\mathrm{CH}_{\text {arom }}\right), 7.63-7.53\left(\mathrm{~m}, 1 \mathrm{H}, \mathrm{CH}_{\text {arom }}\right), 7.50-7.36\left(\mathrm{~m}, 4 \mathrm{H}, \mathrm{CH}_{\text {arom }}\right)$, 7.34-7.27 (m, 3H, $\left.\mathrm{CH}_{\text {arom }}\right), 6.02\left(\mathrm{tt}, 1 \mathrm{H}, J=55.1,4.2 \mathrm{~Hz}, \mathrm{CHF}_{2}\right)$, $5.91-5.81(\mathrm{~m}, 1 \mathrm{H}, \mathrm{H}-3), 5.53(\mathrm{~s}, 1 \mathrm{H}, \mathrm{CHPh}), 5.11(\mathrm{~d}, 1 \mathrm{H}, J=3.6 \mathrm{~Hz}$, $\mathrm{H}-1$ ), 4.33 (dd, $1 \mathrm{H}, J=10.4,4.9 \mathrm{~Hz}, \mathrm{H}-6), 4.07$ (ddd, $1 \mathrm{H}, J=14.8,6.4$, $3.7 \mathrm{~Hz}, \mathrm{H}-5$ ), 3.99-3.77 (m, 4H, H-4, H-6), 3.42 (dd, $1 \mathrm{H}, J=10.4,3.6$ $\mathrm{Hz}, \mathrm{H}-2) .{ }^{13} \mathrm{C}-\mathrm{APT}$ NMR (CDCl, $101 \mathrm{MHz}$, HSQC): $\delta 165.5$ (C= O Bz), $136.8\left(\mathrm{C}_{\mathrm{q}}\right), 133.5,130.1\left(\mathrm{CH}_{\text {arom }}\right), 129.5\left(\mathrm{C}_{\mathrm{q}} \mathrm{Bz}\right), 128.5,128.3$, $126.2\left(\mathrm{CH}_{\text {arom }}\right), 113.7\left(\mathrm{t}, J=241.7 \mathrm{~Hz}, \mathrm{CHF}_{2}\right), 101.8(\mathrm{CHPh}), 99.8$ (C-1), 79.4 (C-4), 69.3 (C-3), $68.7(\mathrm{C}-6), 67.6\left(\mathrm{t}, J=29.0 \mathrm{~Hz}, \mathrm{CH}_{2}-\right.$ $\mathrm{CHF}_{2}$ ), 63.5 (C-5), 61.8 (C-2). Data for the $\beta$-anomer: ${ }^{1} \mathrm{H}$ NMR $\left(\mathrm{CDCl}_{3}, 400 \mathrm{MHz}\right): \delta 5.97$ (tdd, $0.37 \mathrm{H}, J=55.2,4.8,3.5 \mathrm{~Hz}, \mathrm{CHF}_{2}$ ), $5.51(\mathrm{~s}, 0.37 \mathrm{H}, \mathrm{CHPh}), 5.42(\mathrm{t}, 0.37 \mathrm{H}, J=9.8 \mathrm{~Hz}, \mathrm{H}-3), 4.63(\mathrm{~d}$, $0.37 \mathrm{H}, J=7.9 \mathrm{~Hz}, \mathrm{H}-1), 4.38$ (dd, $0.37 \mathrm{H}, J=10.5,5.0 \mathrm{~Hz}, \mathrm{H}-6$ ), 4.12$3.99\left(\mathrm{~m}, 0.37 \mathrm{H}, \mathrm{CHH}-\mathrm{CHF}_{2}\right), 3.98-3.76\left(\mathrm{~m}, 1.11 \mathrm{H}, \mathrm{CHH}-\mathrm{CHF}_{2}\right.$, $\mathrm{H}-4, \mathrm{H}-6), 3.69(\mathrm{dd}, 0.37 \mathrm{H}, J=10.0,7.9 \mathrm{~Hz}, \mathrm{H}-2), 3.58(\mathrm{td}, 0.37 \mathrm{H}, J=$ 9.8, $5.0 \mathrm{~Hz}, \mathrm{H}-5) .{ }^{13} \mathrm{C}-\mathrm{APT}$ NMR $\left(\mathrm{CDCl}_{3}, 101 \mathrm{MHz}\right): \delta 165.4,136.7$, 133.5, 130.0, 129.4, 129.2, 128.6, 126.2, 113.8 ( $\mathrm{t}, J=241.5 \mathrm{~Hz}), 103.1$, 101.7, $78.7(\mathrm{C}-4), 71.5(\mathrm{C}-3), 69.1\left(\mathrm{t}, J=29.0 \mathrm{~Hz}, \mathrm{CH}_{2}-\mathrm{CHF}_{2}\right), 68.4$ (C-6), 66.8 (C-5), 64.9 (C-2). HRMS: $[\mathrm{M}+\mathrm{H}]^{+}$calcd for $\mathrm{C}_{22} \mathrm{H}_{22} \mathrm{~F}_{2} \mathrm{~N}_{3} \mathrm{O}_{6} 462.14712$, found 462.14699 .

Methyl 4-O-(2-Azido-3-O-benzoyl-4,6-O-benzylidene-2-deoxy- $\alpha$ ) $\beta$-D-glucopyranosyl)-2,3,6-tri-O-benzyl- $\beta$-D-galactopyranoside (4H). Donor 4 and acceptor 28 were condensed using the general procedure for $\mathrm{Tf}_{2} \mathrm{O} / \mathrm{Ph}_{2} \mathrm{SO}$-mediated glycosylations (for an additional $18 \mathrm{~h}$ at $-40{ }^{\circ} \mathrm{C}$ ) and purified by flash column chromatography (19:1 to 4:1 pentane/EtOAc) to yield glycosylation product $4 \mathbf{H}(43 \mathrm{mg}, 52 \mu \mathrm{mol}$, 
$52 \%, \alpha / \beta=4: 1)$ as a white solid. $R_{\mathrm{f}} 0.36$ (4:1 pentane/EtOAc). IR (thin film): 698, 737, 997, 1072, 1094, 1265, 1452, 1730, 2106, 2862, 2930. Data for the $\alpha$-anomer: ${ }^{1} \mathrm{H}$ NMR $\left(\mathrm{CDCl}_{3}, 400 \mathrm{MHz}, \mathrm{H}-\mathrm{H}\right.$ COSY, HSQC, HMBC): $\delta 8.12-8.05\left(\mathrm{~m}, 2 \mathrm{H}, \mathrm{CH}_{\text {arom }}\right), 7.57(\mathrm{t}, 1 \mathrm{H}, J$ $\left.=7.4 \mathrm{~Hz}, \mathrm{CH}_{\text {arom }}\right), 7.45\left(\mathrm{t}, 2 \mathrm{H}, J=7.7 \mathrm{~Hz}, \mathrm{CH}_{\text {arom }}\right), 7.42-7.20(\mathrm{~m}$, $\left.20 \mathrm{H}, \mathrm{CH}_{\text {arom }}\right), 5.85\left(\mathrm{t}, 1 \mathrm{H}, \mathrm{J}=9.9 \mathrm{~Hz}, \mathrm{H}-3^{\prime}\right), 5.44(\mathrm{~s}, 1 \mathrm{H}, \mathrm{CHPh}), 5.07$ (d, $\left.1 \mathrm{H}, J=3.9 \mathrm{~Hz}, \mathrm{H}-1^{\prime}\right), 4.93(\mathrm{~d}, 1 \mathrm{H}, J=11.0 \mathrm{~Hz}, \mathrm{CHH} \mathrm{Bn}), 4.84(\mathrm{~d}$, $1 \mathrm{H}, J=10.9 \mathrm{~Hz}, \mathrm{CHH} \mathrm{Bn}), 4.79(\mathrm{~d}, 1 \mathrm{H}, J=12.4 \mathrm{~Hz}, \mathrm{CHH} \mathrm{Bn}), 4.74$ $(\mathrm{d}, 1 \mathrm{H}, J=12.4 \mathrm{~Hz}, \mathrm{CHH} \mathrm{Bn}), 4.55\left(\mathrm{~s}, 2 \mathrm{H}, \mathrm{CH}_{2} \mathrm{Bn}\right), 4.46(\mathrm{td}, 1 \mathrm{H}, J=$ 9.9, $4.9 \mathrm{~Hz}, \mathrm{H}-5^{\prime}$ ), 4.26 (d, $\left.1 \mathrm{H}, J=7.6 \mathrm{~Hz}, \mathrm{H}-1\right), 4.17$ (d, $1 \mathrm{H}, J=3.1$ $\mathrm{Hz}, \mathrm{H}-4), 3.93(\mathrm{t}, 1 \mathrm{H}, J=9.1 \mathrm{~Hz}, \mathrm{H}-6), 3.85(\mathrm{dd}, 1 \mathrm{H}, J=10.2,5.0 \mathrm{~Hz}$, H-6 $\left.{ }^{\prime}\right), 3.81-3.70\left(\mathrm{~m}, 2 \mathrm{H}, \mathrm{H}-2, \mathrm{H}-4^{\prime}\right), 3.64(\mathrm{dd}, 2 \mathrm{H}, J=9.1,5.4 \mathrm{~Hz}, \mathrm{H}-$ 6), 3.57-3.50 (m, $\left.5 \mathrm{H}, \mathrm{CH}_{3} \mathrm{OMe}, \mathrm{H}-5, \mathrm{H}-6^{\prime}\right), 3.47$ (dd, $1 \mathrm{H}, J=10.4$, $\left.3.9 \mathrm{~Hz}, \mathrm{H}-2^{\prime}\right), 3.42(\mathrm{dd}, 1 \mathrm{H}, J=10.0,3.0 \mathrm{~Hz}, \mathrm{H}-3) .{ }^{13} \mathrm{C}-\mathrm{APT}$ NMR $\left(\mathrm{CDCl}_{3}, 101 \mathrm{MHz}, \mathrm{HSQC}, \mathrm{HMBC}\right): \delta 165.3(\mathrm{C}=\mathrm{O} \mathrm{Bz}), 138.7$, 138.3, 137.6, $137.2\left(\mathrm{C}_{\mathrm{q}}\right), 133.3,130.0\left(\mathrm{CH}_{\text {arom }}\right), 129.8\left(\mathrm{C}_{\mathrm{q}}\right), 128.9$, $128.7,128.6,128.6,128.5,128.5,128.4,128.4,128.3,128.2,128.2$, 128.1, 127.9, 127.7, 127.6, 127.6, 126.3, $126.2\left(\mathrm{CH}_{\text {arom }}\right), 105.2(\mathrm{C}-1)$, 101.4 (CHPh), $99.4\left(\mathrm{C}-1^{\prime}\right), 80.0\left(\mathrm{C}-4^{\prime}\right), 79.8(\mathrm{C}-3), 79.0(\mathrm{C}-2), 75.2$ $\left(\mathrm{CH}_{2} \mathrm{Bn}\right), 74.4(\mathrm{C}-4), 73.6,73.2\left(\mathrm{CH}_{2} \mathrm{Bn}\right), 72.6(\mathrm{C}-5), 70.2\left(\mathrm{C}-3^{\prime}\right)$, $68.8\left(\mathrm{C}-6^{\prime}\right), 67.1$ (C-6), 62.8, $62.7\left(\mathrm{C}-2^{\prime}, \mathrm{C}-5^{\prime}\right), 57.0(\mathrm{OMe})$. Diagnostic peaks for the $\beta$-anomer: ${ }^{1} \mathrm{H}$ NMR $\left(\mathrm{CDCl}_{3}, 400 \mathrm{MHz}\right): \delta$ $5.47(\mathrm{~s}, 0.25 \mathrm{H}, \mathrm{CHPh}), 5.34\left(\mathrm{t}, 0.25 \mathrm{H}, J=9.8 \mathrm{~Hz}, \mathrm{H}-3^{\prime}\right), 4.30(\mathrm{~d}$, $\left.0.25 \mathrm{H}, J=7.7 \mathrm{~Hz}, \mathrm{H}-1^{\prime}\right), 4.09$ (d, $\left.0.25 \mathrm{H}, J=2.7 \mathrm{~Hz}, \mathrm{H}-4\right) .{ }^{13} \mathrm{C}-\mathrm{APT}$ NMR $\left(\mathrm{CDCl}_{3}, 101 \mathrm{MHz}\right): \delta 105.1$ (C-1), $102.7\left(\mathrm{C}-1^{\prime}\right), 101.5$ (CHPh), 81.2, 79.6, 78.9, 75.3, 74.6, 73.9, 73.6, 73.3, 71.7, 69.4, 68.5, 66.2, 65.0, 57.3. HRMS: $\left[\mathrm{M}+\mathrm{NH}_{4}\right]^{+}$calcd for $\mathrm{C}_{48} \mathrm{H}_{53} \mathrm{~N}_{4} \mathrm{O}_{11}$ 861.37053, found 861.37067.

Methyl 2-O-(2-Azido-3-O-benzoyl-4,6-O-benzylidene-2-deoxy- $\alpha$ / $\beta$-D-glucopyranosyl)-3-O-benzyl-4,6-O-benzylidene- $\alpha$-D-mannopyranoside (4I). Donor 4 and acceptor 29 were condensed using the general procedure for $\mathrm{Tf}_{2} \mathrm{O} / \mathrm{Ph}_{2} \mathrm{SO}$-mediated glycosylations (for an additional $18 \mathrm{~h}$ at $-40{ }^{\circ} \mathrm{C}$ ) and purified by flash column chromatography (19:1 to $3 / 1$ pentane/EtOAc) to yield glycosylation product $4 \mathrm{I}(55 \mathrm{mg}, 73 \mu \mathrm{mol}, 73 \%, \alpha / \beta=5: 1)$ as a white solid. $R_{\mathrm{f}} 0.36$ (4:1 pentane/EtOAc). IR (thin film): 671, 750, 1037, 1092, 1265, 1373, 1730, 2108, 2913. ${ }^{1} \mathrm{H}$ NMR $\left(\mathrm{CDCl}_{3}, 400 \mathrm{MHz}, \mathrm{H}-\mathrm{H}\right.$ COSY, HSQC, HMBC): $\delta 8.15-8.04\left(\mathrm{~m}, 2 \mathrm{H}, \mathrm{CH}_{\text {arom }}\right), 7.59-7.53(\mathrm{~m}, 1 \mathrm{H}$, $\left.\mathrm{CH}_{\text {arom }}\right), 7.53-7.25\left(\mathrm{~m}, 17 \mathrm{H}, \mathrm{CH}_{\text {arom }}\right), 5.92(\mathrm{dd}, 1 \mathrm{H}, J=10.4,9.5 \mathrm{~Hz}$, $\left.\mathrm{H}-3^{\prime}\right), 5.67(\mathrm{~s}, 1 \mathrm{H}, \mathrm{CHPh}), 5.54\left(\mathrm{~s}, 1 \mathrm{H}, \mathrm{CHPh}^{\prime}\right), 5.51(\mathrm{~d}, 1 \mathrm{H}, J=3.8$ $\left.\mathrm{Hz}, \mathrm{H}-1^{\prime}\right), 4.94$ (d, $\left.1 \mathrm{H}, J=12.2 \mathrm{~Hz}, \mathrm{CHH} \mathrm{Bn}\right), 4.73$ (d, $1 \mathrm{H}, J=1.5$ $\mathrm{Hz}, \mathrm{H}-1), 4.69(\mathrm{~d}, 1 \mathrm{H}, J=12.2 \mathrm{~Hz}, \mathrm{CHH} \mathrm{Bn}), 4.39(\mathrm{t}, 1 \mathrm{H}, J=9.7 \mathrm{~Hz}$, $\mathrm{H}-4), 4.32\left(\mathrm{dd}, 1 \mathrm{H}, J=10.4,4.9 \mathrm{~Hz}, \mathrm{H}-6^{\prime}\right), 4.27$ (dd, $1 \mathrm{H}, J=10.3,4.7$ $\mathrm{Hz}, \mathrm{H}-6), 4.14$ (dd, $1 \mathrm{H}, J=3.1,1.6 \mathrm{~Hz}, \mathrm{H}-2), 4.06-3.99(\mathrm{~m}, 2 \mathrm{H}, \mathrm{H}-3$, $\left.\mathrm{H}-5^{\prime}\right), 3.95(\mathrm{t}, 1 \mathrm{H}, J=10.3 \mathrm{~Hz}, \mathrm{H}-6), 3.86-3.77$ (m, 3H, H-4', H-5, $\left.\mathrm{H}-6^{\prime}\right), 3.38-3.33\left(\mathrm{~m}, 4 \mathrm{H}, \mathrm{CH}_{3} \mathrm{OMe}, \mathrm{H}-2^{\prime}\right) .{ }^{13} \mathrm{C}-\mathrm{APT}$ NMR $\left(\mathrm{CDCl}_{3}\right.$, $101 \mathrm{MHz}, \mathrm{HSQC}): \delta 165.5(\mathrm{C}=\mathrm{O} \mathrm{Bz}), 138.8,137.7,136.8\left(\mathrm{C}_{\mathrm{q}}\right)$, 133.4, $130.1\left(\mathrm{CH}_{\text {arom }}\right), 129.6\left(\mathrm{C}_{\mathrm{q}}\right), 129.2,128.9,128.5,128.4,128.3$, $128.3,128.3,128.3,127.6,127.6,127.4,126.2,126.2,126.2,126.1$ $\left(\mathrm{CH}_{\text {arom }}\right), 101.7,101.6(\mathrm{CHPh}), 100.9(\mathrm{C}-1), 100.1\left(\mathrm{C}-1^{\prime}\right), 79.7(\mathrm{C}-$ $\left.4^{\prime}\right)$, 79.5 (C-4), 75.9, 75.9 (C-2, C-3), $73.6\left(\mathrm{CH}_{2} \mathrm{Bn}\right), 69.2\left(\mathrm{C}-3^{\prime}\right)$, 68.9 (C-6), $68.8\left(\mathrm{C}-6^{\prime}\right), 64.1(\mathrm{C}-5), 63.3\left(\mathrm{C}-5^{\prime}\right), 61.9\left(\mathrm{C}-2^{\prime}\right), 54.9$ (OMe). Diagnostic peaks for the $\beta$-anomer: ${ }^{1} \mathrm{H}$ NMR $\left(\mathrm{CDCl}_{3}, 400\right.$ $\mathrm{MHz}): \delta 5.60(\mathrm{~s}, 0.2 \mathrm{H}, \mathrm{CHPh}), 5.50(\mathrm{~s}, 0.2 \mathrm{H}, \mathrm{CHPh}), 5.40(\mathrm{t}, 0.2 \mathrm{H}, J$ $\left.=9.8 \mathrm{~Hz}, \mathrm{H}-3^{\prime}\right), 4.87(\mathrm{~d}, 0.2 \mathrm{H}, J=1.4 \mathrm{~Hz}, \mathrm{H}-1), 4.80(\mathrm{~d}, 0.2 \mathrm{H}, J=$ $12.3 \mathrm{~Hz}, \mathrm{CHH} \mathrm{Bn}), 3.57$ (td, $0.2 \mathrm{H}, J=9.9,4.3 \mathrm{~Hz}), 3.40\left(\mathrm{~s}, 0.6 \mathrm{H}, \mathrm{CH}_{3}\right.$ OMe). ${ }^{13} \mathrm{C}-\mathrm{APT}$ NMR $\left(\mathrm{CDCl}_{3}, 101 \mathrm{MHz}\right): \delta 101.8\left(\mathrm{C}-1^{\prime}\right), 100.0$ (CHPh), 99.4 (C-1), 78.7, 78.5, 76.4, 74.4, 72.4, 71.5, 68.9, 68.5, 66.9, 65.1, 64.2, 55.2. HRMS: $\left[\mathrm{M}+\mathrm{NH}_{4}\right]^{+}$calcd for $\mathrm{C}_{41} \mathrm{H}_{45} \mathrm{~N}_{4} \mathrm{O}_{11}$ 769.30793 , found 769.30780 .

2,2,2-Trifluoroethyl 2-Azido-3-O-benzoyl-4,6-O-benzylidene-2deoxy- $\alpha$-D-glucopyranoside (4J). Donor 4 and 2,2,2-trifluoroethanol were condensed using the general procedure for $\mathrm{Tf}_{2} \mathrm{O} / \mathrm{Ph}_{2} \mathrm{SO}$ mediated glycosylations (for an additional $30 \mathrm{~min}$ at $-40{ }^{\circ} \mathrm{C}$ ) and purified by flash column chromatography (19:1 to 17:3 pentane/ EtOAc) to yield glycosylation product $4 \mathrm{~J}(41 \mathrm{mg}, 86 \mu \mathrm{mol}, 86 \%, \alpha / \beta$ $>20: 1)$ as a white solid. $R_{\mathrm{f}} 0.15$ (toluene). $[\alpha]_{\mathrm{D}}^{20}=+78.9^{\circ}(c=1.03$, $\mathrm{CHCl}_{3}$ ). IR (thin film): 702, 989, 1085, 1177, 1275, 1717, 2112, 2864. ${ }^{1} \mathrm{H}$ NMR ( $\mathrm{CDCl}_{3}, 400 \mathrm{MHz}, \mathrm{H}-\mathrm{H}$ COSY, HSQC): $\delta 8.13-8.03(\mathrm{~m}$, $\left.2 \mathrm{H}, \mathrm{CH}_{\text {arom }}\right), 7.60-7.53\left(\mathrm{~m}, 1 \mathrm{H}, \mathrm{CH}_{\text {arom }}\right), 7.48-7.38(\mathrm{~m}, 4 \mathrm{H}$,
$\left.\mathrm{CH}_{\text {arom }}\right), 7.33-7.28\left(\mathrm{~m}, 3 \mathrm{H}, \mathrm{CH}_{\text {arom }}\right), 5.87(\mathrm{t}, 1 \mathrm{H}, J=10.0 \mathrm{~Hz}, \mathrm{H}-$ 3), $5.53(\mathrm{~s}, 1 \mathrm{H}, \mathrm{CHPh}), 5.14(\mathrm{~d}, 1 \mathrm{H}, J=3.6 \mathrm{~Hz}, \mathrm{H}-1), 4.33(\mathrm{dd}, 1 \mathrm{H}, J$ $=10.4,4.9 \mathrm{~Hz}, \mathrm{H}-6), 4.14-3.97\left(\mathrm{~m}, 4 \mathrm{H}, \mathrm{CH}_{2} \mathrm{CF}_{3}, \mathrm{H}-5\right), 3.84(\mathrm{t}, 1 \mathrm{H}, \mathrm{J}$ $=9.5 \mathrm{~Hz}, \mathrm{H}-4), 3.80(\mathrm{t}, 1 \mathrm{H}, J=10.3 \mathrm{~Hz}, \mathrm{H}-6), 3.44(\mathrm{dd}, 1 \mathrm{H}, J=10.4$, $3.6 \mathrm{~Hz}, \mathrm{H}-2) .{ }^{13} \mathrm{C}-\mathrm{APT}$ NMR $\left(\mathrm{CDCl}_{3}, 101 \mathrm{MHz}, \mathrm{HSQC}\right): \delta 165.5$ $(\mathrm{C}=\mathrm{O}), 136.7\left(\mathrm{C}_{\mathrm{q}}\right), 133.5,130.0\left(\mathrm{CH}_{\text {arom }}\right), 129.5\left(\mathrm{C}_{\mathrm{q}} \mathrm{Bz}\right), 129.2$, $128.5,127.6,126.2\left(\mathrm{CH}_{\text {arom }}\right), 123.42(\mathrm{q}, J=278.6 \mathrm{~Hz}), 101.8(\mathrm{CHPh})$, 99.9 (C-1), $79.3(\mathrm{C}-4), 69.1(\mathrm{C}-3), 68.6(\mathrm{C}-6), 65.41(\mathrm{q}, J=35.6 \mathrm{~Hz})$, 63.7 (C-5), 61.6 (C-2). HRMS: $\left[\mathrm{M}+\mathrm{NH}_{4}\right]^{+}$calcd for $\mathrm{C}_{22} \mathrm{H}_{24} \mathrm{~F}_{3} \mathrm{~N}_{4} \mathrm{O}_{6}$ 497.16425, found 497.16425.

Ethyl 3-O-Benzyl-4,6-O-benzylidene-2-deoxy-2-(3,5-dinitro-4-pyridone)- $\beta$-D-glucopyranoside (5A). Donor 5 and ethanol were condensed using the general procedure for $\mathrm{Tf}_{2} \mathrm{O} / \mathrm{Ph}_{2} \mathrm{SO}$-mediated glycosylations (for an additional $1 \mathrm{~h}$ at $-40{ }^{\circ} \mathrm{C}$ ) and purified by flash column chromatography (19:1 to $8: 2$ pentane/EtOAc) to yield glycosylation product $\mathbf{5 A}(32 \mathrm{mg}, 59 \mu \mathrm{mol}, 59 \%, \alpha / \beta<1: 20)$ as a yellow solid alongside donor $5(14 \mathrm{mg}) . R_{\mathrm{f}} 0.60$ (7:3 pentane/EtOAc). $[\alpha]_{\mathrm{D}}^{23}=+156.9^{\circ}\left(c=0.64, \mathrm{CHCl}_{3}\right)$. IR (thin film): 698, 998, 1093, $1213,1303,1330,1516,1679,2930 .{ }^{1} \mathrm{H}$ NMR $\left(\mathrm{CDCl}_{3}, 400 \mathrm{MHz}, \mathrm{H}-\right.$ H COSY, HSQC): $\delta 8.58$ (s, $2 \mathrm{H}, \mathrm{CH}$ pyridone), 7.56 (dd, $2 \mathrm{H}, J=7.4$, $\left.2.2 \mathrm{~Hz}, \mathrm{CH}_{\text {arom }}\right), 7.45-7.38\left(\mathrm{~m}, 3 \mathrm{H}, \mathrm{CH}_{\text {arom }}\right), 7.06-6.97(\mathrm{~m}, 5 \mathrm{H}$, $\left.\mathrm{CH}_{\text {arom }}\right), 5.66(\mathrm{~s}, 1 \mathrm{H}, \mathrm{CHPh}), 5.32(\mathrm{~d}, 1 \mathrm{H}, J=8.3 \mathrm{~Hz}, \mathrm{H}-1), 4.70(\mathrm{~d}$, $1 \mathrm{H}, J=11.7 \mathrm{~Hz}, \mathrm{CHH} \mathrm{Bn}$ ), 4.57 (dd, $1 \mathrm{H}, J=10.2,8.7 \mathrm{~Hz}, \mathrm{H}-3), 4.53$ (d, $1 \mathrm{H}, J=11.6 \mathrm{~Hz}, \mathrm{CH} \mathrm{H} \mathrm{Bn),} 4.43$ (dd, $1 \mathrm{H}, J=10.3,4.6 \mathrm{~Hz}, \mathrm{H}-6)$, 3.99-3.81 (m, 4H, CHH Et, H-4, H-5, H-6), 3.72 (dd, 1H, J = 10.3, $8.3 \mathrm{~Hz}, \mathrm{H}-2), 3.60(\mathrm{dq}, 1 \mathrm{H}, J=9.9,7.1 \mathrm{~Hz}, \mathrm{CHH} \mathrm{Et}), 1.08(\mathrm{t}, 3 \mathrm{H}, J=$ $\left.7.1 \mathrm{~Hz}, \mathrm{CH}_{3} \mathrm{Et}\right) .{ }^{13} \mathrm{C}$-APT NMR $\left(\mathrm{CDCl}_{3}, 101 \mathrm{MHz}, \mathrm{HSQC}\right): \delta 160.6$ ( $\mathrm{C}=\mathrm{O}$ pyridone), $141.7\left(\mathrm{C}_{\mathrm{q}} \mathrm{NO}_{2}\right.$ pyridone $), 141.4$ ( $\mathrm{CH}$ pyridone), 137.1, $136.6\left(\mathrm{C}_{\mathrm{q}}\right), 129.4,128.9,128.7,128.5,128.5,126.3\left(\mathrm{CH}_{\text {arom }}\right)$, 101.9 (CHPh), 99.3 (C-1), 82.8 (C-4), 75.4 (C-3), $74.9\left(\mathrm{CH}_{2} \mathrm{Bn}\right)$, 73.8 (C-2), 68.7 (C-6), $66.5\left(\mathrm{CH}_{2} \mathrm{Et}\right), 65.7(\mathrm{C}-5), 15.1\left(\mathrm{CH}_{3} \mathrm{Et}\right)$. HRMS: $[\mathrm{M}+\mathrm{H}]^{+}$calcd for $\mathrm{C}_{27} \mathrm{H}_{28} \mathrm{~N}_{3} \mathrm{O}_{10}$ 554.17692, found 554.17642.

Cyclohexyl 3-O-Benzyl-4,6-O-benzylidene-2-deoxy-2-(3,5-dinitro4-pyridone)- $\beta$-D-glucopyranoside (5B). Donor 5 and cyclohexanol were condensed using the general procedure for $\mathrm{Tf}_{2} \mathrm{O} / \mathrm{Ph}_{2} \mathrm{SO}$ mediated glycosylations (for an additional $1 \mathrm{~h}$ at $-40{ }^{\circ} \mathrm{C}$ ) and purified by flash column chromatography (19:1 to $8: 2$ pentane/ EtOAc) to yield $51 \mathrm{mg}$ of glycosylation product $5 \mathrm{~B}$ as an inseperable mixture with donor 5 (13 mg of 5, $38 \mathrm{mg}$ of $5 \mathrm{~B}, 63 \mu \mathrm{mol}, 63 \%, \alpha / \beta<$ $1: 20)$ as a yellow solid. $R_{\mathrm{f}} 0.75\left(7: 3\right.$ pentane/EtOAc). $R_{\mathrm{f}} 0.55$ (7:3 pentane/EtOAc). IR. (thin film): 697, 718, 749, 789, 910, 997, 1092, 1212, 1302, 1330, 1516, 1623, 1674, 2931. ${ }^{1} \mathrm{H}$ NMR $\left(\mathrm{CDCl}_{3}, 400\right.$ $\mathrm{MHz}, \mathrm{H}-\mathrm{H}$ COSY, HSQC): $\delta 8.60$ (s, 2H, CH pyridone), 7.56 (d, $\left.2 \mathrm{H}, J=4.7 \mathrm{~Hz}, \mathrm{CH}_{\text {arom }}\right), 7.48-7.33\left(\mathrm{~m}, 3 \mathrm{H}, \mathrm{CH}_{\text {arom }}\right), 7.10-6.96(\mathrm{~m}$, $\left.5 \mathrm{H}, \mathrm{CH}_{\text {arom }}\right), 5.65(\mathrm{~s}, 1 \mathrm{H}, \mathrm{CHPh}), 5.41(\mathrm{~d}, 1 \mathrm{H}, J=8.2 \mathrm{~Hz}, \mathrm{H}-1), 4.71$ (d, $1 \mathrm{H}, J=11.7 \mathrm{~Hz}, \mathrm{CHH} \mathrm{Bn}), 4.66-4.50(\mathrm{~m}, 2 \mathrm{H}, \mathrm{CHH} \mathrm{Bn}, \mathrm{H}-3)$, 4.44 (dd, $1 \mathrm{H}, J=10.6,5.2 \mathrm{~Hz}, \mathrm{H}-6), 3.98-3.80$ (m, 3H, H-4, H-5, H6), 3.79-3.61 (m, $2 \mathrm{H}, \mathrm{CH} \mathrm{Cy}, \mathrm{H}-2), 1.91-1.77\left(\mathrm{~m}, 1 \mathrm{H}, \mathrm{CH}_{2} \mathrm{Cy}\right)$, 1.71-1.54 (m, 2H, $\left.\mathrm{CH}_{2} \mathrm{Cy}\right), 1.54-1.45\left(\mathrm{~m}, 1 \mathrm{H}, \mathrm{CH}_{2} \mathrm{Cy}\right), 1.43-0.96$ $\left(\mathrm{m}, 6 \mathrm{H}, \mathrm{CH}_{2} \mathrm{Cy}\right) \cdot{ }^{13} \mathrm{C}$-APT NMR $\left(\mathrm{CDCl}_{3}, 101 \mathrm{MHz}, \mathrm{HSQC}\right): \delta$ $160.5(\mathrm{C}=\mathrm{O}$ pyridone $), 141.6\left(\mathrm{C}_{\mathrm{q}} \mathrm{NO}_{2}\right.$ pyridone $), 141.4(\mathrm{CH}$ pyridone $), 137.1,136.7\left(\mathrm{C}_{\mathrm{q}}\right), 128.8,128.6,128.4,126.3\left(\mathrm{CH}_{\text {arom }}\right)$, 101.9 (CHPh), 98.1 (C-1), 82.7 (C-4), 78.8 (CH Cy), 75.6 (C-3), $74.8\left(\mathrm{CH}_{2} \mathrm{Bn}\right), 74.0$ (C-2), 68.8 (C-6), 65.7 (C-5), 33.3, 31.7, 25.3, 23.9, 23.6 $\left(\mathrm{CH}_{2} \mathrm{Cy}\right)$. HRMS: $[\mathrm{M}+\mathrm{H}]^{+}$calcd for $\mathrm{C}_{31} \mathrm{H}_{34} \mathrm{~N}_{3} \mathrm{O}_{10}$ 608.22387, found 608.22352.

Methyl 6-O-(3-O-Benzyl-4,6-O-benzylidene-2-deoxy-2-(3,5-dinitro-4-pyridone)- $\beta$-D-glucopyranosyl)-2,3,4-tri-O-benzyl- $\alpha$-D-glucopyranoside (3C). Donor 5 and acceptor $\mathbf{2 5}$ were condensed using the general procedure for $\mathrm{Tf}_{2} \mathrm{O} / \mathrm{Ph}_{2} \mathrm{SO}$-mediated glycosylations (for an additional $18 \mathrm{~h}$ at $-40{ }^{\circ} \mathrm{C}$ ) and purified by flash column chromatography (9:1 to $7: 3$ pentane/EtOAc) to yield glycosylation product $5 \mathrm{C}$ (55 mg, $54 \mu \mathrm{mol}, 54 \%, \alpha / \beta<1: 20)$ as a yellow solid. $R_{\mathrm{f}}$ 0.45 (7:3 pentane/EtOAc). $[\alpha]_{\mathrm{D}}^{20}=+90.5^{\circ}\left(c=0.92, \mathrm{CHCl}_{3}\right)$. IR (thin film): $698,1001,1069,1094,1213,1331,1454,1522,1678,2868 .{ }^{1} \mathrm{H}$ NMR $\left(\mathrm{CDCl}_{3}, 400 \mathrm{MHz}, \mathrm{H}-\mathrm{H}\right.$ COSY, HSQC, HMBC): $\delta 8.19$ (s, $2 \mathrm{H}, \mathrm{CH}$ pyridone), 7.54 (dd, $\left.2 \mathrm{H}, J=7.6,2.1 \mathrm{~Hz}, \mathrm{CH}_{\text {arom }}\right), 7.45-7.38$ $\left(\mathrm{m}, 3 \mathrm{H}, \mathrm{CH}_{\text {arom }}\right), 7.34-7.22\left(\mathrm{~m}, 13 \mathrm{H}, \mathrm{CH}_{\text {arom }}\right), 7.20-7.12(\mathrm{~m}, 5 \mathrm{H}$, $\left.\mathrm{CH}_{\text {arom }}\right), 7.04\left(\mathrm{dd}, 2 \mathrm{H}, J=6.6,2.9 \mathrm{~Hz}, \mathrm{CH}_{\text {arom }}\right), 5.66(\mathrm{~s}, 1 \mathrm{H}, \mathrm{CHPh})$, 
$4.92(\mathrm{~d}, 1 \mathrm{H}, J=11.0 \mathrm{~Hz}, \mathrm{CHH} \mathrm{Bn}), 4.85\left(\mathrm{~d}, 1 \mathrm{H}, J=8.3 \mathrm{~Hz}, \mathrm{H}-\mathrm{1}^{\prime}\right)$, 4.83-4.66 (m, 2H, $2 \times \mathrm{CHH} \mathrm{Bn}), 4.72(\mathrm{~d}, 1 \mathrm{H}, J=10.9 \mathrm{~Hz}, \mathrm{CHH}$ $\mathrm{Bn}), 4.69(\mathrm{~d}, 1 \mathrm{H}, J=12.0 \mathrm{~Hz}, \mathrm{CHH} \mathrm{Bn}), 4.60(\mathrm{~d}, 1 \mathrm{H}, J=12.2 \mathrm{~Hz}$, $\mathrm{CH} H \mathrm{Bn}$ ), 4.60 (d, $1 \mathrm{H}, J=12.0 \mathrm{~Hz}, \mathrm{CHH} \mathrm{Bn}), 4.46(\mathrm{~d}, 1 \mathrm{H}, J=3.4$ $\mathrm{Hz}, \mathrm{H}-1$ ), 4.39 (dd, $\left.1 \mathrm{H}, J=10.6,5.0 \mathrm{~Hz}, \mathrm{H}-6^{\prime}\right), 4.34$ (d, $1 \mathrm{H}, J=11.3$ $\mathrm{Hz}, \mathrm{CHH} \mathrm{Bn}), 4.10\left(\mathrm{t}, 1 \mathrm{H}, J=7.9 \mathrm{~Hz}, \mathrm{H}-3^{\prime}\right), 4.01(\mathrm{dd}, 1 \mathrm{H}, J=10.8$, $1.8 \mathrm{~Hz}, \mathrm{H}-6), 3.91(\mathrm{t}, 1 \mathrm{H}, J=9.2 \mathrm{~Hz}, \mathrm{H}-3), 3.89-3.82\left(\mathrm{~m}, 2 \mathrm{H}, \mathrm{H}-4^{\prime}\right.$, H-6), 3.77-3.69 (m, 2H, H-2', H-5), 3.65 (td, $1 \mathrm{H}, J=9.7,5.0 \mathrm{~Hz}, \mathrm{H}-$ $5^{\prime}$ ), 3.52 (dd, $\left.1 \mathrm{H}, J=10.8,7.1 \mathrm{~Hz}, \mathrm{H}-6\right), 3.39$ (dd, $1 \mathrm{H}, J=9.6,3.5 \mathrm{~Hz}$, $\mathrm{H}-2), 3.21$ (s, 3H, $\mathrm{CH}_{3} \mathrm{OMe}$ ), $3.13(\mathrm{dd}, 1 \mathrm{H}, J=9.9,8.9 \mathrm{~Hz}, \mathrm{H}-4)$. ${ }^{13} \mathrm{C}$-APT NMR $\left(\mathrm{CDCl}_{3}, 101 \mathrm{MHz}, \mathrm{HSQC}, \mathrm{HMBC}\right): \delta 159.4(\mathrm{C}=\mathrm{O}$ pyridone), 141.7 ( $\mathrm{C}_{\mathrm{q}} \mathrm{NO}_{2}$ pyridone), 140.2 ( $\mathrm{CH}$ pyridone), 138.6, 138.0, 138.0, 136.7, $135.8\left(\mathrm{C}_{\mathrm{q}}\right), 129.5,129.1,129.0,128.6,128.5$, $128.5,128.5,128.2,128.1,128.0,128.0,127.9,127.7,127.6,126.1$ $\left(\mathrm{CH}_{\text {arom }}\right), 101.8(\mathrm{CHPh}), 100.1\left(\mathrm{C}-1^{\prime}\right), 97.9(\mathrm{C}-1), 82.3\left(\mathrm{C}-4^{\prime}\right), 81.6$ (C-3), 79.8 (C-2), 78.2 (C-4), 75.7, 74.8, 74.3, $\left(\mathrm{CH}_{2} \mathrm{Bn}\right), 74.0$ (C-3'), $73.3\left(\mathrm{CH}_{2} \mathrm{Bn}\right), 72.7\left(\mathrm{C}-2^{\prime}\right), 70.4$ (C-6), $69.3(\mathrm{C}-5), 68.4\left(\mathrm{C}-6^{\prime}\right), 66.1$ $\left(\mathrm{C}-5^{\prime}\right), 55.1(\mathrm{OMe}) .{ }^{13} \mathrm{C}-\mathrm{HMBC}-\mathrm{GATED} N \mathrm{NMR}\left(\mathrm{CDCl}_{3}, 101 \mathrm{MHz}\right)$ : $\delta 100.1\left(J=163 \mathrm{~Hz}, \mathrm{C}-1^{\prime}\right)$. HRMS: $[\mathrm{M}+\mathrm{H}]^{+}$calcd for $\mathrm{C}_{53} \mathrm{H}_{54} \mathrm{~N}_{3} \mathrm{O}_{15}$ 972.35494, found 972.35546 .

2-Fluoroethyl 3-O-Benzyl-4,6-O-benzylidene-2-deoxy-2-(3,5-dinitro-4-pyridone)- $\beta$-D-glucopyranoside (5D). Donor 5 and 2 -fluoroethanol were condensed using the general procedure for $\mathrm{Tf}_{2} \mathrm{O} / \mathrm{Ph}_{2} \mathrm{SO}$ mediated glycosylations (for an additional $1 \mathrm{~h}$ at $-40{ }^{\circ} \mathrm{C}$ ) and purified by flash column chromatography (19:1 to $8: 2$ pentane/EtOAc) to yield glycosylation product $5 \mathrm{D}(24 \mathrm{mg}, 43 \mu \mathrm{mol}, 43 \%, \alpha / \beta<1: 20)$ as a yellow solid alongside donor $5(15.6 \mathrm{mg}) . R_{\mathrm{f}} 0.42$ (3/2 pentane/ EtOAc). $[\alpha]_{\mathrm{D}}^{23}=+142.9^{\circ}\left(c=0.48, \mathrm{CHCl}_{3}\right)$. IR (thin film): 698, 752, $1070,1096,1213,1304,1331,1518,1680,2870,3064 .{ }^{1} \mathrm{H}$ NMR $\left(\mathrm{CDCl}_{3}, 400 \mathrm{MHz}, \mathrm{H}-\mathrm{H}\right.$ COSY, HSQC $): \delta 8.58(\mathrm{~s}, 2 \mathrm{H}, \mathrm{CH}$ pyridone), 7.63-7.49 (m, 2H, $\left.\mathrm{CH}_{\text {arom }}\right), 7.47-7.36\left(\mathrm{~m}, 3 \mathrm{H}, \mathrm{CH}_{\text {arom }}\right)$, $7.09-6.96\left(\mathrm{~m}, 5 \mathrm{H}, \mathrm{CH}_{\text {arom }}\right), 5.66(\mathrm{~s}, 1 \mathrm{H}, \mathrm{CHPh}), 5.46(\mathrm{~d}, 1 \mathrm{H}, J=8.3$ $\mathrm{Hz}, \mathrm{H}-1), 4.71$ (d, $1 \mathrm{H}, J=11.7 \mathrm{~Hz}, \mathrm{CHH} \mathrm{Bn}), 4.57$ (dd, $1 \mathrm{H}, J=10.3$, $8.7 \mathrm{~Hz}, \mathrm{H}-3), 4.53$ (d, $1 \mathrm{H}, J=11.7 \mathrm{~Hz}, \mathrm{CHH} \mathrm{Bn}), 4.48-4.42(\mathrm{~m}, 2 \mathrm{H}$, CHHF, H-6), $4.33(\mathrm{t}, 1 \mathrm{H}, J=4.1 \mathrm{~Hz}, \mathrm{CHHF}), 4.09-3.81(\mathrm{~m}, 5 \mathrm{H}$, $\left.\mathrm{CH}_{2}-\mathrm{CH}_{2} \mathrm{~F}, \mathrm{H}-4, \mathrm{H}-5, \mathrm{H}-6\right), 3.77$ (dd, $1 \mathrm{H}, J=10.3,8.4 \mathrm{~Hz}, \mathrm{H}-2$ ). ${ }^{13} \mathrm{C}-\mathrm{APT}$ NMR $\left(\mathrm{CDCl}_{3}, 101 \mathrm{MHz}, \mathrm{HSQC}\right): \delta 160.6(\mathrm{C}=\mathrm{O}$ pyridone), 141.5, $141.5\left(\mathrm{C}_{\mathrm{q}} \mathrm{NO}_{2}, \mathrm{CH}\right.$ pyridone $), 137.0,136.6\left(\mathrm{C}_{\mathrm{q}}\right)$, 129.4, 129.0, 128.7, 128.6, 128.5, $126.3\left(\mathrm{CH}_{\text {arom }}\right), 101.9(\mathrm{CHPh}), 99.8$ (C-1), 82.7 (C-4), 82.5 (d, $\left.J=169.4 \mathrm{~Hz}, \mathrm{CH}_{2} \mathrm{~F}\right), 75.3$ (C-3), 74.9 $\left(\mathrm{CH}_{2} \mathrm{Bn}\right), 73.6(\mathrm{C}-2), 69.5\left(\mathrm{~d}, J=19.5 \mathrm{~Hz}, \mathrm{CH}_{2}-\mathrm{CH}_{2} \mathrm{~F}\right), 68.6(\mathrm{C}-6)$, 65.8 (C-5). HRMS: $[\mathrm{M}+\mathrm{H}]^{+}$calcd for $\mathrm{C}_{27} \mathrm{H}_{27} \mathrm{FN}_{3} \mathrm{O}_{10}$ 572.16760, found 572.16705 .

Methyl 4-O-(3-O-Benzyl-4,6-O-benzylidene-2-deoxy-2-(3,5-dinitro-4-pyridone)- $\beta$-D-glucopyranosyl)-2,3,6-tri-O-benzyl- $\alpha$-D-glucopyranoside (5E). Donor 5 and acceptor 26 were condensed using the general procedure for $\mathrm{Tf}_{2} \mathrm{O} / \mathrm{Ph}_{2} \mathrm{SO}$-mediated glycosylations (for an additional $18 \mathrm{~h}$ at $-40{ }^{\circ} \mathrm{C}$ ) and purified by flash column chromatography (9:1 to $7: 3$ pentane/EtOAc) to yield glycosylation product $5 \mathrm{E}(54 \mathrm{mg}, 56 \mu \mathrm{mol}, 56 \%, \alpha / \beta<1: 20)$ as a yellow solid. $R_{\mathrm{f}}$ $0.37\left(7: 3\right.$ pentane/EtOAc). $[\alpha]_{\mathrm{D}}^{23}=+83.3^{\circ}\left(c=0.84, \mathrm{CHCl}_{3}\right)$. IR (thin film): 696, 734, 997, 1028, 1039, 1092, 1209, 1302, 1327, 1454, 1522, 1682, 2862, 2900, 3030, 3065. ${ }^{1} \mathrm{H}$ NMR $\left(\mathrm{CDCl}_{3}, 500 \mathrm{MHz}, \mathrm{H}-\mathrm{H}\right.$ COSY, HSQC, HMBC, NOESY): $\delta 7.74$ (s, $2 \mathrm{H}, \mathrm{CH}$ pyridone), $7.57-$ $7.53\left(\mathrm{~m}, 2 \mathrm{H}, \mathrm{CH}_{\text {arom }}\right), 7.49-7.38\left(\mathrm{~m}, 8 \mathrm{H}, \mathrm{CH}_{\text {arom }}\right), 7.36-7.25(\mathrm{~m}$, $\left.13 \mathrm{H}, \mathrm{CH}_{\text {arom }}\right), 7.04-7.00\left(\mathrm{~m}, 2 \mathrm{H}, \mathrm{CH}_{\text {arom }}\right), 5.57(\mathrm{~s}, 1 \mathrm{H}, \mathrm{CHPh}), 4.90$ $(\mathrm{d}, 1 \mathrm{H}, J=11.7 \mathrm{~Hz}, \mathrm{CHH} \mathrm{Bn}), 4.77(\mathrm{~d}, 1 \mathrm{H}, J=12.1 \mathrm{~Hz}, \mathrm{CHH} \mathrm{Bn})$, $4.74(\mathrm{~d}, 1 \mathrm{H}, J=12.3 \mathrm{~Hz}, \mathrm{CHH} \mathrm{Bn}), 4.69$ (d, $1 \mathrm{H}, J=11.7 \mathrm{~Hz}, \mathrm{CHH}$ $\mathrm{Bn}), 4.66(\mathrm{~d}, 1 \mathrm{H}, J=12.0 \mathrm{~Hz}, \mathrm{CHH} \mathrm{Bn}), 4.63(\mathrm{~d}, 1 \mathrm{H}, J=12.1 \mathrm{~Hz}$, CHH Bn), 4.56 (d, $1 \mathrm{H}, J=12.3 \mathrm{~Hz}, \mathrm{CHH} \mathrm{Bn}), 4.54$ (d, $1 \mathrm{H}, J=3.6$ $\mathrm{Hz}, \mathrm{H}-1$ ), 4.35 (d, 1H, J = 8.2 Hz, H-1' ), 4.27-4.20 (m, 2H, CHH Bn, $\left.\mathrm{H}-6^{\prime}\right), 3.92(\mathrm{t}, 1 \mathrm{H}, J=9.5 \mathrm{~Hz}, \mathrm{H}-4), 3.70(\mathrm{t}, 1 \mathrm{H}, J=9.3 \mathrm{~Hz}, \mathrm{H}-3), 3.67$ $\left(\mathrm{t}, 1 \mathrm{H}, J=9.0 \mathrm{~Hz}, \mathrm{H}-4^{\prime}\right), 3.58\left(\mathrm{t}, 1 \mathrm{H}, J=10.4 \mathrm{~Hz}, \mathrm{H}-6^{\prime}\right), 3.53-3.45$ $\left(\mathrm{m}, 2 \mathrm{H}, \mathrm{H}-2, \mathrm{H}-3^{\prime}\right), 3.43$ (dd, $\left.1 \mathrm{H}, J=11.4,1.5 \mathrm{~Hz}, \mathrm{H}-6\right), 3.40-3.34$ (m, 1H, H-5), $3.31\left(\mathrm{~s}, 2 \mathrm{H}, \mathrm{CH}_{3} \mathrm{OMe}\right), 3.18(\mathrm{dd}, 1 \mathrm{H}, J=10.5,8.3 \mathrm{~Hz}$, H-2'), 3.04 (dd, $1 \mathrm{H}, J=11.3,2.6 \mathrm{~Hz}, \mathrm{H}-6), 2.92(\mathrm{td}, 1 \mathrm{H}, J=9.8,5.1$ $\left.\mathrm{Hz}, \mathrm{H}-5^{\prime}\right) .{ }^{13} \mathrm{C}-\mathrm{APT}$ NMR $\left(\mathrm{CDCl}_{3}, 126 \mathrm{MHz}, \mathrm{HSQC}, \mathrm{HMBC}\right): \delta$ 159.3 ( $\mathrm{C}=\mathrm{O}$ pyridone $), 141.8\left(\mathrm{C}_{\mathrm{q}} \mathrm{NO}_{2}\right.$ pyridone $), 139.6(\mathrm{CH}$ pyridone), 139.4, 138.1, 137.8, 136.8, $135.7\left(\mathrm{C}_{\mathrm{q}}\right), 129.6,129.3,129.2$, 129.0, 128.9, 128.6, 128.6, 128.5, 128.4, 128.1, 128.1, 127.8, 126.1
$\left(\mathrm{CH}_{\text {arom }}\right), 101.8$ (CHPh), $98.1(\mathrm{C}-1), 97.6\left(\mathrm{C}-1^{\prime}\right), 82.4\left(\mathrm{C}-4^{\prime}\right), 79.7$ (C-2), 79.2 (C-3), $75.3\left(\mathrm{CH}_{2} \mathrm{Bn}\right), 74.4(\mathrm{C}-4), 73.9,73.6,73.5\left(\mathrm{CH}_{2}\right.$ $\mathrm{Bn}), 73.0$ (C-3'), $72.5\left(\mathrm{C}-2^{\prime}\right), 69.2(\mathrm{C}-5), 68.4\left(\mathrm{C}-6^{\prime}\right), 68.1(\mathrm{C}-6)$, $65.6\left(\mathrm{C}-5^{\prime}\right), 55.7$ (OMe). HRMS: $[\mathrm{M}+\mathrm{H}]^{+}$calcd for $\mathrm{C}_{53} \mathrm{H}_{54} \mathrm{~N}_{3} \mathrm{O}_{15}$ 972.35494, found 972.35519 .

Methyl (Methyl 4-O-[3-O-Benzyl-4,6-O-benzylidene-2-deoxy-2(3,5-dinitro-4-pyridone)- $\alpha / \beta$-D-glucopyranosyl]-2,3-di-O-benzyl- $\alpha$-Dglucopyranosyl uronate) (5F). Donor 5 and acceptor 27 were condensed using the general procedure for $\mathrm{Tf}_{2} \mathrm{O} / \mathrm{Ph}_{2} \mathrm{SO}$-mediated glycosylations (for an additional $18 \mathrm{~h}$ at $-40{ }^{\circ} \mathrm{C}$ ) and purified by flash column chromatography (9:1 to 7:3 pentane/EtOAc) to yield glycosylation product $5 \mathrm{~F}(27 \mathrm{mg}, 30 \mu \mathrm{mol}, 30 \%, \alpha / \beta=1: 3.6)$ as a yellow solid. $R_{\mathrm{f}} 0.51$ (7:3 pentane/EtOAc). IR (thin film): 648, 698, 733, 789, 910, 995, 1090, 1171, 1209, 1302, 1331, 1454, 1520, 1678, 1744, 2932. Data for the $\beta$-anomer: ${ }^{1} \mathrm{H}$ NMR $\left(\mathrm{CDCl}_{3}, 500 \mathrm{MHz}, \mathrm{H}-\right.$ H COSY, HSQC, HMBC): $\delta 8.06(\mathrm{~s}, 2 \mathrm{H}, \mathrm{CH}$ pyridone), 7.50 (dd, $\left.2 \mathrm{H}, J=7.3,2.0 \mathrm{~Hz}, \mathrm{CH}_{\text {arom }}\right), 7.45-7.35\left(\mathrm{~m}, 6 \mathrm{H}, \mathrm{CH}_{\text {arom }}\right), 7.33-7.18$ $\left(\mathrm{m}, 9 \mathrm{H}, \mathrm{CH}_{\text {arom }}\right), 7.03\left(\mathrm{dd}, 2 \mathrm{H}, J=6.9,2.2 \mathrm{~Hz}, \mathrm{CH}_{\text {arom }}\right), 6.96(\mathrm{dd}, 1 \mathrm{H}$, $\left.J=14.5,6.9 \mathrm{~Hz}, \mathrm{CH}_{\text {arom }}\right), 5.55(\mathrm{~s}, 1 \mathrm{H}, \mathrm{CHPh}), 5.17(\mathrm{~d}, 1 \mathrm{H}, J=8.2 \mathrm{~Hz}$, $\left.\mathrm{H}-1^{\prime}\right), 4.92(\mathrm{~d}, 1 \mathrm{H}, J=11.3 \mathrm{~Hz}, \mathrm{CHH} \mathrm{Bn}), 4.82-4.72(\mathrm{~m}, 3 \mathrm{H}, \mathrm{CHH}$ $\mathrm{Bn}, 2 \times \mathrm{CHH} \mathrm{Bn}), 4.61-4.55(\mathrm{~m}, 2 \mathrm{H}, 2 \times \mathrm{CHH} \mathrm{Bn}), 4.51(\mathrm{~d}, 1 \mathrm{H}, J=$ $3.3 \mathrm{~Hz}, \mathrm{H}-1), 4.14$ (dd, $\left.1 \mathrm{H}, J=10.6,4.8 \mathrm{~Hz}, \mathrm{H}-6^{\prime}\right), 3.97-3.88(\mathrm{~m}, 2 \mathrm{H}$, H-3', $\mathrm{H}-4), 3.83$ (d, $1 \mathrm{H}, J=9.7 \mathrm{~Hz}, \mathrm{H}-5), 3.82-3.73$ (m, $2 \mathrm{H}, \mathrm{H}-3, \mathrm{H}-$ $\left.4^{\prime}\right)$, 3.54-3.43 (m, 6H, CH $\left.\mathrm{CO}_{2} \mathrm{Me}, \mathrm{H}-2, \mathrm{H}-2^{\prime}, \mathrm{H}-5^{\prime}\right)$, 3.42-3.36 (m, $\left.4 \mathrm{H}, \mathrm{CH}_{3} \mathrm{OMe}, \mathrm{H}-6^{\prime}\right) .{ }^{13} \mathrm{C}-\mathrm{APT}$ NMR $\left(\mathrm{CDCl}_{3}, 126 \mathrm{MHz}, \mathrm{HSQC}\right.$, HMBC): $\delta 170.0\left(\mathrm{C}=\mathrm{O} \mathrm{CO} \mathrm{CO}_{2} \mathrm{Me}\right), 159.5(\mathrm{C}=\mathrm{O}$ pyridone $), 141.5$ ( $\mathrm{C}_{\mathrm{q}} \mathrm{NO}_{2}$ pyridone), 140.4 ( $\mathrm{CH}$ pyridone), 139.1, 137.8, 136.7, 135.7 $\left(C_{\mathrm{q}}\right), 129.5,129.2,129.2,129.1,129.0,128.9,128.7,128.7,128.6$, $128.5,128.4,128.2,128.2,127.7,127.7,126.1,126.0,125.7\left(\mathrm{CH}_{\text {arom }}\right)$, 101.7 (CHPh), 98.8 (C-1'), 98.3 (C-1), 82.3 (C-4'), 79.1 (C-3), 78.8 (C-2), 77.8 (C-4), 75.5, $74.2\left(\mathrm{CH}_{2} \mathrm{Bn}\right), 74.0\left(\mathrm{C}-3^{\prime}\right), 73.7\left(\mathrm{CH}_{2} \mathrm{Bn}\right)$, $72.9\left(\mathrm{C}-2^{\prime}\right), 68.8(\mathrm{C}-5), 68.2\left(\mathrm{C}-6^{\prime}\right), 65.9\left(\mathrm{C}-5^{\prime}\right), 56.1(\mathrm{OMe}), 52.9$ $\left(\mathrm{CO}_{2} \mathrm{Me}\right) .{ }^{13} \mathrm{C}-\mathrm{HMBC}-\mathrm{GATED}$ NMR $\left(\mathrm{CDCl}_{3}, 101 \mathrm{MHz}\right): \delta 98.8(\mathrm{~J}$ $\left.=167 \mathrm{~Hz}, \mathrm{C}-1^{\prime}\right)$. Diagnostic peaks for the $\alpha$-anomer: ${ }^{1} \mathrm{H}$ NMR $\left(\mathrm{CDCl}_{3}, 500 \mathrm{MHz}, \mathrm{H}-\mathrm{H}\right.$ COSY, HSQC): $\delta 5.74(\mathrm{~d}, 0.28 \mathrm{H}, J=3.9$ $\left.\mathrm{Hz}, \mathrm{H}_{-1} \mathrm{1}^{\prime}\right), 5.61(\mathrm{~s}, 0.28 \mathrm{H}, \mathrm{CHPh}), 5.03(\mathrm{~d}, 0.28 \mathrm{H}, J=12.7 \mathrm{~Hz}, \mathrm{CHH}$ $\mathrm{Bn}), 4.70(\mathrm{~d}, 0.28 \mathrm{H}, J=12.3 \mathrm{~Hz}), 4.62(\mathrm{~d}, 0.28 \mathrm{H}, J=12.2 \mathrm{~Hz}, \mathrm{CHH}$ $\mathrm{Bn}), 4.46(\mathrm{~d}, 0.28 \mathrm{H}, J=12.3 \mathrm{~Hz}, \mathrm{CHH} \mathrm{Bn}), 4.37$ (dd, $0.28 \mathrm{H}, J=10.5$, $\left.4.9 \mathrm{~Hz}, \mathrm{H}-6^{\prime}\right), 4.29(\mathrm{~d}, 0.28 \mathrm{H}, J=9.9 \mathrm{~Hz}, \mathrm{H}-5), 4.08(\mathrm{t}, 0.28 \mathrm{H}, J=9.4$ $\mathrm{Hz}$ ), 3.69 (dd, $\left.0.28 \mathrm{H}, J=10.6,3.9 \mathrm{~Hz}, \mathrm{H}-2^{\prime}\right) .{ }^{13} \mathrm{C}-\mathrm{APT} \mathrm{NMR}\left(\mathrm{CDCl}_{3}\right.$, $126 \mathrm{MHz}$, HSQC): $\delta 169.7,159.2,141.2,101.8(\mathrm{CHPh}), 96.8\left(\mathrm{C}-1^{\prime}\right)$, 82.6, 80.7, 79.8, 74.5, 74.4, 73.1, 72.2, 69.6, 69.5, 68.1, 63.0, 56.1, 53.3. ${ }^{13} \mathrm{C}-\mathrm{HMBC}-\mathrm{GATED}$ NMR $\left(\mathrm{CDCl}_{3}, 101 \mathrm{MHz}\right): \delta 96.8(J=181 \mathrm{~Hz}$, C-1' $\left.{ }^{\prime}\right)$. HRMS: $[\mathrm{M}+\mathrm{H}]^{+}$calcd for $\mathrm{C}_{47} \mathrm{H}_{48} \mathrm{~N}_{3} \mathrm{O}_{16}$ 910.30291, found 910.30315 .

2,2-Difluoroethyl 3-O-Benzyl-4,6-O-benzylidene-2-deoxy-2-(3,5dinitro-4-pyridone)- $\alpha / \beta$-D-glucopyranoside (5G). Donor 5 and 2,2difluoroethanol were condensed using the general procedure for $\mathrm{Tf}_{2} \mathrm{O} / \mathrm{Ph}_{2} \mathrm{SO}$-mediated glycosylations (for an additional $1 \mathrm{~h}$ at -40 ${ }^{\circ} \mathrm{C}$ ) and purified by flash column chromatography (19:1 to $8: 2$ pentane/EtOAc) to yield glycosylation product 5 G $(17.3 \mathrm{mg}, 29 \mu \mathrm{mol}$ of $\alpha$-anomer; $17.8 \mathrm{mg}, 30 \mu \mathrm{mol}$ of $\beta$-anomer; $\alpha / \beta=1: 1 ; 59 \%)$ as a yellow solid alongside donor $5(11 \mathrm{mg}) . R_{\mathrm{f}} 0.12$ and 0.30 (7:3 pentane/EtOAc). IR (thin film): 698, 997, 1069, 1094, 1211, 1300, $1339,1520,1684,2922$. Data for the $\alpha$-anomer: ${ }^{1} \mathrm{H}$ NMR (acetone- $d_{6}$, $400 \mathrm{MHz}, \mathrm{H}-\mathrm{H}$ COSY, HSQC): $\delta 8.91$ (s, $2 \mathrm{H}, \mathrm{CH}$ pyridone), 7.61$7.53\left(\mathrm{~m}, 2 \mathrm{H}, \mathrm{CH}_{\text {arom }}\right), 7.48-7.37\left(\mathrm{~m}, 3 \mathrm{H}, \mathrm{CH}_{\text {arom }}\right), 7.25-7.10(\mathrm{~m}, 5 \mathrm{H}$, $\left.\mathrm{CH}_{\text {arom }}\right), 6.16\left(\mathrm{tt}, 1 \mathrm{H}, J=55.0,3.7 \mathrm{~Hz}, \mathrm{CHF}_{2}\right), 5.83(\mathrm{~s}, 1 \mathrm{H}, \mathrm{CHPh})$, $5.56(\mathrm{~d}, 1 \mathrm{H}, J=3.7 \mathrm{~Hz}, \mathrm{H}-1), 4.91(\mathrm{~d}, 1 \mathrm{H}, J=11.9 \mathrm{~Hz}, \mathrm{CHH} \mathrm{Bn})$ 4.79 (dd, $1 \mathrm{H}, J=10.7,3.7 \mathrm{~Hz}, \mathrm{H}-2), 4.71-4.62(\mathrm{~m}, 2 \mathrm{H}, \mathrm{CHH} \mathrm{Bn}, \mathrm{H}-$ 3), $4.37(\mathrm{dd}, 1 \mathrm{H}, J=10.1,4.9 \mathrm{~Hz}, \mathrm{H}-6), 4.18-4.06(\mathrm{~m}, 2 \mathrm{H}, \mathrm{CHH}-$ $\left.\mathrm{CHF}_{2}, \mathrm{H}-5\right), 4.03(\mathrm{dd}, 1 \mathrm{H}, J=9.6,8.6 \mathrm{~Hz}, \mathrm{H}-4), 3.96-3.83(\mathrm{~m}, 2 \mathrm{H}$, $\left.\mathrm{CHH}-\mathrm{CHF}_{2}, \mathrm{H}-6\right) .{ }^{13} \mathrm{C}-\mathrm{APT}$ NMR (acetone- $\left.d_{6}, 101 \mathrm{MHz}, \mathrm{HSQC}\right)$ : $\delta 160.0$ ( $\mathrm{C}=\mathrm{O}$ pyridone), $142.9\left(\mathrm{C}_{\mathrm{q}} \mathrm{NO}_{2}\right.$ pyridone $), 142.6(\mathrm{CH}$ pyridone), 138.6, $138.5\left(\mathrm{C}_{\mathrm{q}}\right), 129.8,129.2,129.0,129.0,128.9,127.0$ $\left(\mathrm{CH}_{\text {arom }}\right), 115.0\left(\mathrm{t}, J=239.2 \mathrm{~Hz}, \mathrm{CHF}_{2}\right), 102.1(\mathrm{CHPh}), 98.8(\mathrm{C}-1)$, 83.5 (C-4), $75.0\left(\mathrm{CH}_{2} \mathrm{Bn}\right), 74.7$ (C-3), 69.9 (C-2), 68.9 (C-6), 67.93 $\left(\mathrm{t}, J=27.3 \mathrm{~Hz}, \mathrm{CH}_{2}-\mathrm{CHF}_{2}\right), 63.9(\mathrm{C}-5)$. Data for the $\beta$-anomer: ${ }^{1} \mathrm{H}$ NMR ( $\mathrm{CDCl}_{3}, 400 \mathrm{MHz}, \mathrm{H}-\mathrm{H}$ COSY, HSQC): $\delta 8.67(\mathrm{~s}, 2 \mathrm{H}, \mathrm{CH}$ pyridone), $7.60-7.52\left(\mathrm{~m}, 2 \mathrm{H}, \mathrm{CH}_{\text {arom }}\right), 7.46-7.37$ (m, $3 \mathrm{H}, \mathrm{CH}_{\text {arom }}$ ), 
7.03-6.93 (m, 5H, $\left.\mathrm{CH}_{\text {arom }}\right), 5.75\left(\mathrm{tt}, 1 \mathrm{H}, J=54.9,3.9 \mathrm{~Hz}, \mathrm{CHF}_{2}\right)$, $5.65(\mathrm{~s}, 1 \mathrm{H}, \mathrm{CHPh}), 5.59(\mathrm{~d}, 1 \mathrm{H}, J=8.3 \mathrm{~Hz}, \mathrm{H}-1), 4.76-4.64(\mathrm{~m}, 2 \mathrm{H}$, CHH Bn, H-3), 4.50 (d, 1H, $J=11.6 \mathrm{~Hz}, \mathrm{CHH} \mathrm{Bn}$ ), 4.45 (dd, $1 \mathrm{H}, J=$ $10.4,4.9 \mathrm{~Hz}, \mathrm{H}-6), 4.05$ (td, $1 \mathrm{H}, J=9.7,5.0 \mathrm{~Hz}, \mathrm{H}-5), 4.00-3.80(\mathrm{~m}$, $\left.4 \mathrm{H}, \mathrm{CH}_{2}-\mathrm{CHF}_{2}, \mathrm{H}-4, \mathrm{H}-6\right), 3.77$ (dd, $1 \mathrm{H}, J=10.3,8.4 \mathrm{~Hz}, \mathrm{H}-2$ ). ${ }^{13} \mathrm{C}$-APT NMR $\left(\mathrm{CDCl}_{3}, 101 \mathrm{MHz}, \mathrm{HSQC}\right): \delta 160.9(\mathrm{C}=\mathrm{O}$ pyridone), 141.7 ( $\mathrm{C}_{\mathrm{q}} \mathrm{NO}_{2}$ pyridone), 141.5 ( $\mathrm{CH}$ pyridone), 137.0, $136.7\left(\mathrm{C}_{\mathrm{q}}\right), 129.4,128.8,128.6,128.5,128.4,126.4\left(\mathrm{CH}_{\text {arom }}\right), 113.4(\mathrm{t}$, $J=241.5 \mathrm{~Hz}, 102.0(\mathrm{CHPh}), 99.9(\mathrm{C}-1), 82.6(\mathrm{C}-4), 75.5(\mathrm{C}-3), 75.1$ $\left(\mathrm{CH}_{2} \mathrm{Bn}\right), 73.7(\mathrm{C}-2), 68.9\left(\mathrm{t}, J=27.8 \mathrm{~Hz}, \mathrm{CH}_{2}-\mathrm{CHF}_{2}\right), 68.6(\mathrm{C}-6)$, 65.8 (C-5). HRMS: $[\mathrm{M}+\mathrm{H}]^{+}$calcd for $\mathrm{C}_{27} \mathrm{H}_{26} \mathrm{~F}_{2} \mathrm{~N}_{3} \mathrm{O}_{10}$ 590.15808, found 590.15741 .

Methyl 4-O-(3-O-Benzyl-4,6-O-benzylidene-2-deoxy-2-(3,5-dinitro-4-pyridone)- $\beta$-D-glucopyranosyl)-2,3,6-tri-O-benzyl- $\beta$-D-galactopyranoside (5H). Donor 5 and acceptor 28 were condensed using the general procedure for $\mathrm{Tf}_{2} \mathrm{O} / \mathrm{Ph}_{2} \mathrm{SO}$-mediated glycosylations (for an additional $18 \mathrm{~h}$ at $-40{ }^{\circ} \mathrm{C}$ ) and purified by flash column chromatography (9:1 to 7:3 pentane/EtOAc) to yield glycosylation product $5 \mathrm{H}(51 \mathrm{mg}, 52 \mu \mathrm{mol}, 52 \%, \alpha / \beta<1: 20)$ as a yellow solid. $R_{f}$ $0.49\left(7: 3\right.$ pentane/EtOAc). $[\alpha]_{\mathrm{D}}^{20}=+35.5^{\circ}\left(c=0.85, \mathrm{CHCl}_{3}\right)$. IR (thin film ): 698, 750, 999, 1072, 1094, 1213, 1454, 1522, 1682, 2868. ${ }^{1} \mathrm{H}$ NMR $\left(\mathrm{CDCl}_{3}, 400 \mathrm{MHz}, \mathrm{H}-\mathrm{H}\right.$ COSY, HSQC, HMBC): $\delta 8.20$ (s, $2 \mathrm{H}, \mathrm{CH}$ pyridone), $7.55-7.49\left(\mathrm{~m}, 2 \mathrm{H}, \mathrm{CH}_{\text {arom }}\right), 7.47-7.40(\mathrm{~m}, 3 \mathrm{H}$, $\left.\mathrm{CH}_{\text {arom }}\right), 7.40-7.15\left(\mathrm{~m}, 18 \mathrm{H}, \mathrm{CH}_{\text {arom }}\right), 7.07-7.00\left(\mathrm{~m}, 2 \mathrm{H}, \mathrm{CH}_{\text {arom }}\right)$, $5.63(\mathrm{~s}, 1 \mathrm{H}, \mathrm{CHPh}), 5.03\left(\mathrm{~d}, 1 \mathrm{H}, J=8.3 \mathrm{~Hz}, \mathrm{H}-1^{\prime}\right), 4.80(\mathrm{~d}, 1 \mathrm{H}, J=$ $12.2 \mathrm{~Hz}, \mathrm{CHH} \mathrm{Bn}), 4.64(\mathrm{~d}, 1 \mathrm{H}, J=10.4 \mathrm{~Hz}, \mathrm{CHH} \mathrm{Bn}), 4.61(\mathrm{~d}, 1 \mathrm{H}, J$ $=12.3 \mathrm{~Hz}, \mathrm{CHH} \mathrm{Bn}), 4.57(\mathrm{~d}, 1 \mathrm{H}, J=12.2 \mathrm{~Hz}, \mathrm{CHH} \mathrm{Bn}), 4.50(\mathrm{~s}, 2 \mathrm{H}$, $\left.\mathrm{CH}_{2} \mathrm{Bn}\right), 4.47(\mathrm{~d}, 1 \mathrm{H}, J=12.3 \mathrm{~Hz}, \mathrm{CHH} \mathrm{Bn}), 4.29(\mathrm{~d}, 1 \mathrm{H}, J=10.4$ $\mathrm{Hz}, \mathrm{CHH} \mathrm{Bn}$ ), 4.20 (dd, $1 \mathrm{H}, J=10.5,5.0 \mathrm{~Hz}, \mathrm{H}-6^{\prime}$ ), 4.13 (d, $1 \mathrm{H}, J=$ $7.6 \mathrm{~Hz}, \mathrm{H}-1), 3.91$ (d, $1 \mathrm{H}, J=2.6 \mathrm{~Hz}, \mathrm{H}-4), 3.88-3.78$ (m, $2 \mathrm{H}, \mathrm{H}-3^{\prime}$, $\left.\mathrm{H}-4^{\prime}\right), 3.74\left(\mathrm{t}, 1 \mathrm{H}, J=10.3 \mathrm{~Hz}, \mathrm{H}-6^{\prime}\right), 3.70(\mathrm{dd}, 1 \mathrm{H}, J=9.9,8.4 \mathrm{~Hz}, \mathrm{H}-$ $\left.2^{\prime}\right)$, 3.66-3.52 (m, 2H, H-6, H-6), 3.46 (s, 4H, $\left.\mathrm{CH}_{3} \mathrm{OMe}, \mathrm{H}-5\right)$, 3.41-3.33 (m, 2H, H-3, H-5'), 2.93 (dd, $1 \mathrm{H}, J=9.6,7.6 \mathrm{~Hz}, \mathrm{H}-2)$. ${ }^{13} \mathrm{C}$-APT NMR $\left(\mathrm{CDCl}_{3}, 101 \mathrm{MHz}, \mathrm{HSQC}, \mathrm{HMBC}\right): \delta 159.4\left(\mathrm{C}_{\mathrm{q}}\right.$ pyridone), 141.6 ( $\mathrm{C}_{\mathrm{q}} \mathrm{NO}{ }_{2}$ pyridone), 140.5 ( $\mathrm{CH}$ pyridone), 138.2, $138.0,137.6,136.6,135.6\left(\mathrm{C}_{\mathrm{q}}\right), 129.6,129.3,129.1,129.1,128.9$, $128.7,128.6,128.6,128.4,128.4,128.1,128.0,127.7,127.5,126.1$ $\left(\mathrm{CH}_{\text {arom }}\right), 104.8(\mathrm{C}-1), 101.8(\mathrm{CHPh}), 99.6\left(\mathrm{C}-1^{\prime}\right), 82.3$ (C-4'), 80.2, 80.2 (C-2, C-3), $75.4\left(\mathrm{CH}_{2} \mathrm{Bn}\right), 74.6(\mathrm{C}-4), 74.5,74.4\left(\mathrm{CH}_{2} \mathrm{Bn}\right), 74.2$ $\left(\mathrm{C}-3^{\prime}\right), 73.5\left(\mathrm{CH}_{2} \mathrm{Bn}\right), 72.5$ (C-5), $72.3\left(\mathrm{C}-2^{\prime}\right), 68.6(\mathrm{C}-6), 68.2(\mathrm{C}-$ $\left.6^{\prime}\right), 66.2\left(\mathrm{C}-5^{\prime}\right), 57.3$ (OMe). ${ }^{13} \mathrm{C}-\mathrm{HMBC}-\mathrm{GATED}$ NMR $\left(\mathrm{CDCl}_{3}\right.$, $101 \mathrm{MHz}): \delta 104.8(J=159 \mathrm{~Hz}, \mathrm{C}-1), 99.6\left(J=165 \mathrm{~Hz}, \mathrm{C}-1^{\prime}\right)$. HRMS: $[\mathrm{M}+\mathrm{H}]^{+}$calcd for $\mathrm{C}_{53} \mathrm{H}_{54} \mathrm{~N}_{3} \mathrm{O}_{15}$ 972.35494, found 972.35542

Methyl 2-O-(3-O-Benzyl-4,6-O-benzylidene-2-deoxy-2-(3,5-dinitro-4-pyridone)- $\alpha / \beta$-D-glucopyranosyl)-3-O-benzyl-4,6-O-benzylidene- $\alpha$-D-mannopyranoside (5I). Donor 5 and acceptor 29 were condensed using the general procedure for $\mathrm{Tf}_{2} \mathrm{O} / \mathrm{Ph}_{2} \mathrm{SO}$-mediated glycosylations (for an additional $18 \mathrm{~h}$ at $-40{ }^{\circ} \mathrm{C}$ ) and purified by flash column chromatography (9:1 to $7: 3$ pentane/EtOAc) to yield glycosylation product $5 \mathrm{I}(47 \mathrm{mg}, 53 \mu \mathrm{mol}, 53 \%, \alpha / \beta=1: 1.3)$ as a yellow solid. $R_{\mathrm{f}} 0.34$ and 0.49 (7:3 pentane/EtOAc). IR: (thin film): 646, 696, 731, 789, 908, 997, 1090, 1123, 1211, 1302, 1333, 1454, $1518,1624,1674,2910$. Data reported for a $0.8: 1$ mixture of anomers: ${ }^{1} \mathrm{H}$ NMR ( $\mathrm{CDCl}_{3}, 400 \mathrm{MHz}, \mathrm{H}-\mathrm{H}$ COSY, HSQC, HMBC): $\delta 8.48(\mathrm{~s}$, $2 \mathrm{H}$, pyridone $\left.{ }_{\beta}\right), 8.33\left(\mathrm{~s}, 1.6 \mathrm{H}\right.$, pyridone $\left.{ }_{\alpha}\right), 7.58-7.26(\mathrm{~m}, 22.8 \mathrm{H}$, $\left.\mathrm{CH}_{\text {arom }}\right), 7.23-7.01\left(\mathrm{~m}, 11.8 \mathrm{H}, \mathrm{CH}_{\text {arom }}\right), 6.99-6.94(\mathrm{~m}, 1.6 \mathrm{H}$, $\left.\mathrm{CH}_{\text {arom }}\right), 5.67\left(\mathrm{~s}, 1.8 \mathrm{H}, \mathrm{CHPh}_{\alpha}{ }_{\alpha}, \mathrm{CHPh}^{\prime}{ }^{\prime}\right), 5.62\left(\mathrm{~s}, 0.8 \mathrm{H}, \mathrm{CHPh}_{\alpha}\right)$, $5.53\left(\mathrm{~s}, 1 \mathrm{H}, \mathrm{CHPh}_{\beta}\right), 5.27\left(\mathrm{~d}, 0.8 \mathrm{H}, J=3.9 \mathrm{~Hz}, \mathrm{H}_{-1}{ }_{\alpha}{ }_{\alpha}\right), 5.24(\mathrm{~d}, 1 \mathrm{H}, J$ $\left.=8.3 \mathrm{~Hz}, \mathrm{H}-1^{\prime}{ }_{\beta}\right), 4.84\left(\mathrm{~d}, 1 \mathrm{H}, J=12.1 \mathrm{~Hz}, \mathrm{CHH} \mathrm{Bn}{ }_{\beta}\right), 4.79(\mathrm{~d}, 0.8 \mathrm{H}, J$ $\left.=12.2 \mathrm{~Hz}, \mathrm{CHH} \mathrm{Bn}{ }_{\alpha}\right), 4.75\left(\mathrm{~d}, 1 \mathrm{H}, J=12.1 \mathrm{~Hz}, \mathrm{CHH} \mathrm{Bn}{ }_{\beta}\right), 4.70(\mathrm{~d}$ $\left.1 \mathrm{H}, J=12.2 \mathrm{~Hz}, \mathrm{CHH} \mathrm{Bn} \mathrm{Bn}_{\beta}\right), 4.67\left(\mathrm{~d}, 0.8 \mathrm{H}, J=1.1 \mathrm{~Hz}, \mathrm{H}-1_{\alpha}\right), 4.64(\mathrm{~d}$, $\left.1 \mathrm{H}, J=12.1 \mathrm{~Hz}, \mathrm{CH} H \mathrm{Bn}_{\beta}\right), 4.57\left(\mathrm{~d}, 0.8 \mathrm{H}, J=12.2 \mathrm{~Hz}, \mathrm{CHH} \mathrm{Bn} \mathrm{B}_{\alpha}\right.$, $4.52\left(\mathrm{~d}, 0.8 \mathrm{H}, J=11.1 \mathrm{~Hz}, \mathrm{CH} H \mathrm{Bn}_{\alpha}\right), 4.50(\mathrm{dd}, 1 \mathrm{H}, J=10.3,8.2 \mathrm{~Hz}$, $\left.\mathrm{H}-3^{\prime}{ }_{\beta}\right), 4.46-4.41\left(\mathrm{~m}, 1 \mathrm{H}, \mathrm{H}-6^{\prime}{ }_{\beta}\right), 4.33(\mathrm{~d}, 0.8 \mathrm{H}, J=11.1 \mathrm{~Hz}, \mathrm{CHH}$ $\mathrm{Bn}_{\alpha}$ ), 4.34-4.26 (m, 1.6H, H- $\left.6_{\alpha}, \mathrm{H}-6_{\alpha}^{\prime}\right), 4.22-4.15$ (m, $2.8 \mathrm{H}, \mathrm{H}-1_{\beta}$, $\left.\mathrm{H}-2_{\beta}, \mathrm{H}-3^{\prime}{ }_{\alpha}\right), 4.10-3.96\left(\mathrm{~m}, 4.4 \mathrm{H}, \mathrm{H}-2_{\alpha}, \mathrm{H}-2^{\prime}{ }_{\alpha}, \mathrm{H}-2^{\prime}{ }_{\beta}, \mathrm{H}-5^{\prime}{ }_{\alpha}, \mathrm{H}-6_{\beta}\right)$, 3.95-3.83 (m, 8.2 $\mathrm{H}, \mathrm{H}-3_{\alpha}, \mathrm{H}-3_{\beta}, \mathrm{H}-4_{\beta}, \mathrm{H}-4^{\prime}{ }_{\alpha}, \mathrm{H}-4^{\prime}{ }_{\beta}, \mathrm{H}-5^{\prime}{ }_{\beta}, \mathrm{H}-6_{\alpha}, \mathrm{H}-$ $\left.6^{\prime}{ }_{\alpha}, \mathrm{H}-6^{\prime}{ }_{\beta}\right), 3.81-3.74\left(\mathrm{~m}, 1.6 \mathrm{H}, \mathrm{H}-4_{\alpha}, \mathrm{H}-5_{\alpha}\right), 3.61(\mathrm{dq}, 1 \mathrm{H}, J=9.0$, $\left.4.5 \mathrm{~Hz}, \mathrm{H}-5_{\beta}\right), 3.50\left(\mathrm{t}, 1 \mathrm{H}, J=10.3 \mathrm{~Hz}, \mathrm{H}-6_{\beta}\right), 3.38\left(\mathrm{~s}, 2.4 \mathrm{H}, \mathrm{CH}_{3}\right.$
$\left.\mathrm{OMe}_{\alpha}\right), 3.15$ (s, 3H, $\left.\mathrm{CH}_{3} \mathrm{OMe}_{\beta}\right) .{ }^{13} \mathrm{C}-\mathrm{APT} \mathrm{NMR}\left(\mathrm{CDCl}_{3}, 101 \mathrm{MHz}\right.$ HSQC, HMBC): $\delta$ 159.9, $159.7(\mathrm{C}=\mathrm{O}$ pyridone), $142.1(\mathrm{CH}$ pyridone $\left._{\alpha}\right), 141.7\left(\mathrm{C}_{\mathrm{q}} \mathrm{NO}_{2}\right.$ pyridone $), 140.9\left(\mathrm{CH}\right.$ pyridone $\left.{ }_{\beta}\right), 140.8$ $\left(\mathrm{C}_{\mathrm{q}} \mathrm{NO}_{2}\right.$ pyridone $), 138.2,137.6,137.4,137.4,136.8,136.6,136.5$, $136.0\left(\mathrm{C}_{\mathrm{q}}\right), 129.6,129.4,129.1,129.0,128.9,128.8,128.7,128.6$, $128.5,128.4,128.4,128.3,128.3,127.8,127.8,127.6,126.2,126.1$, $126.1\left(\mathrm{CH}_{\text {arom }}\right), 101.9,101.8\left(\mathrm{CHPh}_{\alpha, \beta}^{\prime}\right), 101.6,101.6\left(\mathrm{CHPh}_{\alpha, \beta}\right)$, $100.7\left(\mathrm{C}-1_{\alpha}\right), 99.8\left(\mathrm{C}-1_{\alpha}^{\prime}{ }_{\alpha}\right), 99.2\left(\mathrm{C}-1_{\beta}\right), 98.8\left(\mathrm{C}-1^{\prime}{ }_{\beta}\right), 83.1\left(\mathrm{C}-4_{\alpha}^{\prime}{ }_{\alpha}\right)$, $82.4\left(\mathrm{C}-4^{\prime}{ }_{\beta}\right), 79.6\left(\mathrm{C}-4_{\alpha}\right), 79.1\left(\mathrm{C}-2_{\alpha}\right), 78.5\left(\mathrm{C}-4_{\beta}\right), 76.1\left(\mathrm{C}-2_{\beta}\right), 75.0$ $\left(\mathrm{C}-3_{\alpha}\right), 74.5,74.5,74.3\left(\mathrm{CH}_{2} \mathrm{Bn}\right), 74.2\left(\mathrm{C}-3_{\beta}, \mathrm{C}-3^{\prime}{ }_{\beta}\right), 72.9\left(\mathrm{C}-2^{\prime}{ }_{\beta}\right)$, $72.7\left(\mathrm{CH}_{2} \mathrm{Bn}\right), 72.5\left(\mathrm{C}-3^{\prime}{ }_{\alpha}\right), 69.9\left(\mathrm{C}-2^{\prime}{ }_{\alpha}\right), 68.5,68.5,68.4\left(\mathrm{C}-6_{\alpha, \beta}, \mathrm{C}-\right.$ $\left.6^{\prime}{ }_{\alpha \beta}\right), 66.1\left(\mathrm{C}-5^{\prime}{ }_{\beta}\right), 63.7\left(\mathrm{C}-5_{\beta}\right), 63.3\left(\mathrm{C}-5_{\alpha}\right), 63.1\left(\mathrm{C}-5^{\prime}{ }_{\alpha}\right), 55.1,55.1$ (OMe). ${ }^{13} \mathrm{C}$-HMBC-GATED NMR $\left(\mathrm{CDCl}_{3}, 101 \mathrm{MHz}\right): \delta 99.8(\mathrm{~J}=$ $\left.176 \mathrm{~Hz}, \mathrm{C}-1^{\prime}{ }_{\alpha}\right), 98.8\left(\mathrm{~J}=164 \mathrm{~Hz}, \mathrm{C}-1^{\prime}{ }_{\beta}\right)$. HRMS: $[\mathrm{M}+\mathrm{H}]^{+}$calcd for $\mathrm{C}_{46} \mathrm{H}_{46} \mathrm{~N}_{3} \mathrm{O}_{15} 880.29234$, found 880.29252.

2,2,2-Trifluoroethyl 3-O-Benzyl-4,6-O-benzylidene-2-deoxy-2(3,5-dinitro-4-pyridone)- $\alpha / \beta$-D-glucopyranoside (5J). Donor 5 and 2,2,2-trifluoroethanol were condensed using the general procedure for $\mathrm{Tf}_{2} \mathrm{O} / \mathrm{Ph}_{2} \mathrm{SO}$-mediated glycosylations (for an additional $1 \mathrm{~h}$ at -40 ${ }^{\circ} \mathrm{C}$ ) and purified by flash column chromatography (19:1 to 8:2 pentane/EtOAc) to yield glycosylation product $5 \mathrm{~J}(28 \mathrm{mg}, 46 \mu \mathrm{mol}$ of $\alpha$-anomer; $7 \mathrm{mg}, 12 \mu \mathrm{mol}$ of $\beta$-anomer; $\alpha / \beta=4: 1 ; 58 \%)$ as a yellow solid alongside glucal $24(4 \mathrm{mg})$ and donor $5(13 \mathrm{mg}) . R_{\mathrm{f}} 0.15$ and 0.67 (7:3 pentane/EtOAc). IR (thin film): 698, 754, 1001, 1071, 1096, $1169,1215,1279,1304,1331,1520,1680,2855,2924,3065$. Data for the $\alpha$-anomer: ${ }^{1} \mathrm{H}$ NMR (acetone- $d_{6}, 400 \mathrm{MHz}, \mathrm{H}-\mathrm{H}$ COSY, HSQC): $\delta 8.92\left(\mathrm{~s}, 2 \mathrm{H}, \mathrm{CH}\right.$ pyridone), $7.60-7.54\left(\mathrm{~m}, 2 \mathrm{H}, \mathrm{CH}_{\text {arom }}\right)$, 7.47-7.37 (m, 3H, CH $\left.\mathrm{CH}_{\text {arom }}\right), 7.24-7.13\left(\mathrm{~m}, 5 \mathrm{H}, \mathrm{CH}_{\text {arom }}\right), 5.84(\mathrm{~s}, 1 \mathrm{H}$, $\mathrm{CHPh}), 5.65(\mathrm{~d}, 1 \mathrm{H}, J=3.7 \mathrm{~Hz}, \mathrm{H}-1), 4.92(\mathrm{~d}, 1 \mathrm{H}, J=11.8 \mathrm{~Hz}, \mathrm{CHH}$ Bn), $4.84(\mathrm{dd}, 1 \mathrm{H}, J=10.7,3.7 \mathrm{~Hz}, \mathrm{H}-2), 4.73(\mathrm{dd}, 1 \mathrm{H}, J=10.7,8.4$ $\mathrm{Hz}, \mathrm{H}-3), 4.70(\mathrm{~d}, 1 \mathrm{H}, J=11.8 \mathrm{~Hz}, \mathrm{CHH} \mathrm{Bn}), 4.51-4.38(\mathrm{~m}, 1 \mathrm{H}$, $\left.\mathrm{CHH}-\mathrm{CF}_{3}\right), 4.38(\mathrm{dd}, 1 \mathrm{H}, J=10.1,4.7 \mathrm{~Hz}, \mathrm{H}-6), 4.30-4.17(\mathrm{~m}, 1 \mathrm{H}$, $\left.\mathrm{CHH}-\mathrm{CF}_{3}\right), 4.12(\mathrm{dd}, 1 \mathrm{H}, J=9.8,4.7 \mathrm{~Hz}, \mathrm{H}-5), 4.09-4.02(\mathrm{~m}, 1 \mathrm{H}$, $\mathrm{H}-4), 3.93(\mathrm{t}, 1 \mathrm{H}, J=10.0 \mathrm{~Hz}, \mathrm{H}-6) .{ }^{13} \mathrm{C}-\mathrm{APT}$ NMR (acetone- $d_{6}, 101$ $\mathrm{MHz}, \mathrm{HSQC}): \delta 160.0$ (C=O pyridone), $142.9\left(\mathrm{C}_{\mathrm{q}} \mathrm{NO}_{2}\right.$ pyridone), $142.6\left(\mathrm{CH}\right.$ pyridone), 138.6, $138.5\left(\mathrm{C}_{\mathrm{q}}\right), 129.8,129.2,129.0,129.0$, $128.9,127.0\left(\mathrm{CH}_{2}\right), 124.72\left(\mathrm{q}, J=277.4 \mathrm{~Hz}, \mathrm{CF}_{3}\right), 102.1(\mathrm{CHPh})$, 98.8 (C-1), 83.4 (C-4), $75.1\left(\mathrm{CH}_{2} \mathrm{Bn}\right), 74.6$ (C-3), 69.8 (C-2), 68.8 (C-6), $65.84\left(\mathrm{q}, J=35.0 \mathrm{~Hz}, \mathrm{CH}_{2}-\mathrm{CF}_{3}\right), 64.1$ (C-5). Diagnostic peaks for the $\beta$-anomer: ${ }^{1} \mathrm{H}$ NMR $\left(\mathrm{CDCl}_{3}, 400 \mathrm{MHz}, \mathrm{H}-\mathrm{H}\right.$ COSY, HSQC): $\delta 8.61$ (s, $2 \mathrm{H}, \mathrm{CH}$ pyridone), $7.56(\mathrm{dd}, 2 \mathrm{H}, J=6.6,2.9 \mathrm{~Hz}$, $\left.\mathrm{CH}_{\text {arom }}\right), 7.41\left(\mathrm{dd}, 3 \mathrm{H}, J=5.0,1.7 \mathrm{~Hz}, \mathrm{CH}_{\text {arom }}\right), 7.00\left(\mathrm{~s}, 5 \mathrm{H}, \mathrm{CH}_{\text {arom }}\right)$, $5.66(\mathrm{~s}, 1 \mathrm{H}, \mathrm{CHPh}), 5.58(\mathrm{~d}, 1 \mathrm{H}, J=8.3 \mathrm{~Hz}, \mathrm{H}-1), 4.75-4.62(\mathrm{~m}, 2 \mathrm{H}$, CHH Bn, H-3), $4.52(\mathrm{~d}, 1 \mathrm{H}, J=11.7 \mathrm{~Hz}, \mathrm{CHH} \mathrm{Bn}), 4.46(\mathrm{dd}, 1 \mathrm{H}, J=$ 10.5, $4.8 \mathrm{~Hz}, \mathrm{H}-6)$, 4.17-3.99 (m, 3H, $\mathrm{CH}_{2}-\mathrm{CF}_{3}, \mathrm{H}-5$ ), 3.91-3.82 (m, $2 \mathrm{H}, \mathrm{H}-4, \mathrm{H}-6), 3.78$ (dd, $1 \mathrm{H}, J=10.0,8.5 \mathrm{~Hz}, \mathrm{H}-2)$. HRMS: [M + $\mathrm{H}]^{+}$calcd for $\mathrm{C}_{27} \mathrm{H}_{25} \mathrm{~F}_{3} \mathrm{~N}_{3} \mathrm{O}_{10} 608.14865$, found 608.14825 .

\section{ASSOCIATED CONTENT}

\section{Supporting Information}

The Supporting Information is available free of charge on the ACS Publications website at DOI: 10.1021/acs.joc.7b00470.

NMR spectra of all new compounds, decomposition study and identification of anomeric triflates by NMR spectroscopy, ${ }^{1} \mathrm{H}$ NMR spectra of isolated disaccharide fractions of competition experiments (PDF)

\section{AUTHOR INFORMATION}

\section{Corresponding Author}

*E-mail: jcodee@chem.leidenuniv.nl

ORCID

Jeroen D. C. Codée: 0000-0003-3531-2138

Notes

The authors declare no competing financial interest. 


\section{REFERENCES}

(1) Capila, I.; Linhardt, R. J. Angew. Chem., Int. Ed. 2002, 41 (3), 390-412.

(2) Spillmann, D.; Lindahl, U. Curr. Opin. Struct. Biol. 1994, 4 (5), 677-682.

(3) Griffiths, A. J.; Davies, D. B. Carbohydr. Polym. 1991, 14 (3), 241-279.

(4) Griffiths, A. J.; Davies, D. B. Carbohydr. Polym. 1991, 14 (4), 339-365.

(5) Boltje, T. J.; Buskas, T.; Boons, G.-J. Nat. Chem. 2009, 1 (8), 611-622.

(6) Christina, A. E.; van der Marel, G. A.; Codée, J. D. C. In Modern Synthetic Methods in Carbohydrate Chemistry; Werz, D. B., Vidal, S., Eds.; Wiley-VCH Verlag GmbH \& Co. KGaA: Weinheim, Germany, 2013; pp 97-124.

(7) Nigudkar, S. S.; Demchenko, A. V. Chem. Sci. 2015, 6 (5), 26872704.

(8) Bennett, C. S. Selective Glycosylations: Synthetic Methods and Catalysts; Wiley-VCH Verlag GmbH: Weinheim, Germany, 2017.

(9) Crich, D.; Cai, W. J. Org. Chem. 1999, 64 (13), 4926-4930.

(10) Crich, D.; Sun, S. J. Am. Chem. Soc. 1997, 119 (46), 1121711223

(11) Crich, D.; Sun, S. J. Org. Chem. 1996, 61 (14), 4506-4507.

(12) Huang, M.; Garrett, G. E.; Birlirakis, N.; Bohé, L.; Pratt, D. A.; Crich, D. Nat. Chem. 2012, 4 (8), 663-667.

(13) Crich, D.; Chandrasekera, N. S. Angew. Chem., Int. Ed. 2004, 43 (40), 5386-5389.

(14) Adero, P. O.; Furukawa, T.; Huang, M.; Mukherjee, D.; Retailleau, P.; Bohé, L.; Crich, D. J. Am. Chem. Soc. 2015, 137 (32), 10336-10345.

(15) Huang, M.; Furukawa, T.; Retailleau, P.; Crich, D.; Bohé, L. Carbohydr. Res. 2016, 427, 21-28.

(16) Bohé, L.; Crich, D. Carbohydr. Res. 2015, 403, 48-59.

(17) Huang, M.; Retailleau, P.; Bohé, L.; Crich, D. J. Am. Chem. Soc. 2012, 134 (36), 14746-14749.

(18) Bongat, A. F. G.; Demchenko, A. V. Carbohydr. Res. 2007, 342 (3-4), 374-406.

(19) For the use of C2-N-C3-O-carbamate-protected glucosamines, see, for example: ref 6 and Codée, J. D. C.; Ali, A.; Overkleeft, H. S.; van der Marel, G. A. C. R. Chim. 2011, 14 (2-3), 178-193.

(20) Cid, M. B.; Alfonso, F.; Martín-Lomas, M. Chem. - Eur. J. 2005, 11 (3), 928-938.

(21) Li, J.; Dai, Y.; Li, W.; Laval, S.; Xu, P.; Yu, B. Asian J. Org. Chem. 2015, 4 (8), 756-762.

(22) van der Vorm, S.; Hansen, T.; Overkleeft, H. S.; van der Marel, G. A.; Codee, J. D. C. Chem. Sci. 2017, 8 (3), 1867-1875.

(23) Hansch, C.; Leo, A.; Taft, R. W. Chem. Rev. 1991, 91 (2), 165195.

(24) Ammer, J.; Mayr, H. J. Phys. Org. Chem. 2013, 26 (1), 59-63.

(25) Minegishi, S.; Kobayashi, S.; Mayr, H. J. Am. Chem. Soc. 2004, 126 (16), 5174-5181.

(26) Beaver, M. G.; Woerpel, K. A. J. Org. Chem. 2010, 75 (4), $1107-1118$.

(27) Krumper, J. R.; Salamant, W. A.; Woerpel, K. A. J. Org. Chem. 2009, 74 (21), 8039-8050.

(28) Hagen, B.; Ali, S.; Overkleeft, H. S.; van der Marel, G. A.; Codée, J. D. C. J. Org. Chem. 2017, 82 (2), 848-868.

(29) Matsumura, E.; Ariga, M.; Tohda, Y.; Kawashima, T. Tetrahedron Lett. 1981, 22 (8), 757-758.

(30) Matsumura, E.; Kobayashi, H.; Nishikawa, T.; Ariga, M.; Tohda, Y.; Kawashima, T. Bull. Chem. Soc. Jpn. 1984, 57 (7), 1961-1965.

(31) Lindberg, J.; Öhberg, L.; Garegg, P. J.; Konradsson, P. Tetrahedron 2002, 58 (7), 1387-1398.

(32) Emmadi, M.; Kulkarni, S. S. J. Org. Chem. 2011, 76 (11), 47034709 .

(33) Coutant, C.; Jacquinet, J.-C. J. Chem. Soc., Perkin Trans. 1 1995, No. $12,1573-1581$.

(34) Goddard-Borger, E. D.; Stick, R. V. Org. Lett. 2007, 9 (19), $3797-3800$
(35) Chiara, J. L.; García, Á.; Cristóbal-Lumbroso, G. J. Org. Chem. 2005, 70 (10), 4142-4151.

(36) Trans-amination by double Michael addition-elimination with a nucleophilic amine ensures deprotection. Typical conditions included $0.2 \mathrm{M}$ solution of DNPY-protected compound in pyridine at room temperature treated with amine ( 2 equiv) and stirred for $30 \mathrm{~min}$. Extractive workup gave deprotected amines in excellent yield (>90\%). Efficient nucleophilic amines are ethylenediamine (DNPY product is water-soluble), propylamine, hexylamine, and hydrazine.

(37) Frihed, T. G.; Bols, M.; Pedersen, C. M. Chem. Rev. 2015, 115 (11), 4963-5013.

(38) Codée, J. D. C.; Litjens, R. E. J. N.; den Heeten, R.; Overkleeft, H. S.; van Boom, J. H.; van der Marel, G. A. Org. Lett. 2003, 5 (9), 1519-1522.

(39) The chemical shifts of the resonances in donor 5 are very sensitive to temperature, concentration, and chemical environment. It was ensured that the sample had not precipitated during the lowtemperature NMR experiments at all temperatures; the cause of the line broadening is therefore attributed to the poor solvation and mobility of the donor in dichloromethane.

(40) Lee, J.-C.; Greenberg, W. A.; Wong, C.-H. Nat. Protoc. 2007, 1 (6), 3143-3152.

(41) Douglas, N. L.; Ley, S. V.; Lücking, U.; Warriner, S. L. J. Chem. Soc., Perkin Trans. 1 1998, No. 1, 51-66.

(42) Zhang, Z.; Ollmann, I. R.; Ye, X.-S.; Wischnat, R.; Baasov, T.; Wong, C.-H. J. Am. Chem. Soc. 1999, 121 (4), 734-753.

(43) Pedersen, C. M.; Marinescu, L. G.; Bols, M. Chem. Commun. 2008, No. 21, 2465-2467.

(44) Kamkhachorn, T.; Parameswar, A. R.; Demchenko, A. V. Org. Lett. 2010, 12 (13), 3078-3081.

(45) Walvoort, M. T. C.; de Witte, W.; van Dijk, J.; Dinkelaar, J.; Lodder, G.; Overkleeft, H. S.; Codée, J. D. C.; van der Marel, G. A. Org. Lett. 2011, 13 (16), 4360-4363.

(46) de Jong, A.-R.; Hagen, B.; van der Ark, V.; Overkleeft, H. S.; Codée, J. D. C.; Van der Marel, G. A. J. Org. Chem. 2012, 77 (1), 108125.

(47) Mootoo, D. R.; Konradsson, P.; Udodong, U.; Fraser-Reid, B. J. Am. Chem. Soc. 1988, 110 (16), 5583-5584.

(48) Andrews, C. W.; Rodebaugh, R.; Fraser-Reid, B. J. Org. Chem. 1996, 61 (16), 5280-5289.

(49) Fraser-Reid, B.; López, J. C. In Reactivity Tuning in Oligosaccharide Assembly; Fraser-Reid, B., Cristóbal López, J., Eds.; Springer: Berlin, 2011; pp 1-29.

(50) Balmond, E. I.; Coe, D. M.; Galan, M. C.; McGarrigle, E. M. Angew. Chem., Int. Ed. 2012, 51 (36), 9152-9155.

(51) Ennis, S. C.; Cumpstey, I.; Fairbanks, A. J.; Butters, T. D.; Mackeen, M.; Wormald, M. R. Tetrahedron 2002, 58 (46), 94039411.

(52) Ingle, A. B.; Chao, C.-S.; Hung, W.-C.; Mong, K.-K. T. Org. Lett. 2013, 15 (20), 5290-5293.

(53) The moderate yields obtained in glycosylations with donor $\mathbf{5}$ are attributed to the unidentified baseline (TLC) material when the reaction was activated and stirred at $-40^{\circ} \mathrm{C}$. For model acceptors 610 , the donor was activated to $-50{ }^{\circ} \mathrm{C}$, and unreacted donor was consistently recovered.

(54) Crich, D.; Li, L. J. Org. Chem. 2007, 72 (5), 1681-1690.

(55) Whitfield, D. M. Carbohydr. Res. 2007, 342 (12-13), 17261740.

(56) Hosoya, T.; Kosma, P.; Rosenau, T. Carbohydr. Res. 2015, 411, 64-69.

(57) Smith, D. M.; Woerpel, K. A. Org. Biomol. Chem. 2006, 4 (7), 1195-1201.

(58) Crich, D.; Cai, W.; Dai, Z. J. Org. Chem. 2000, 65 (5), 12911297.

(59) This striking stereodirecting effect has been hypothesized to be caused by remote participation, but direct evidence for this type of participation on benzylidene mannose donors has not yet been found. Other stereoelectronic stabilizing effects might be responsible for the apparent stereoselectivity and await further study. For a discussion on 
remote participation, see: (a) Crich, D.; Hu, T.; Cai, F. J. Org. Chem. 2008, 73 (22), 8942-8953. (b) Baek, J. Y.; Lee, B.-Y.; Jo, M. G.; Kim, K. S. J. Am. Chem. Soc. 2009, 131 (48), 17705-17713. (c) Komarova, B. S.; Orekhova, M. V.; Tsvetkov, Y. E.; Nifantiev, N. E. Carbohydr. Res. 2014, 384, 70-86.

(60) Crich, D.; Smith, M.; Yao, Q.; Picione, J. Synthesis 2001, 2001 (02), 0323-0326.

(61) Benakli, K.; Zha, C.; Kerns, R. J. J. Am. Chem. Soc. 2001, 123 (38), 9461-9462.

(62) Buskas, T.; Garegg, P. J.; Konradsson, P.; Maloisel, J.-L. Tetrahedron: Asymmetry 1994, 5 (11), 2187-2194.

(63) Emmadi, M.; Kulkarni, S. S. J. Org. Chem. 2011, 76 (11), 47034709

(64) You, F.; Twieg, R. J. Tetrahedron Lett. 1999, 40 (50), 87598762. 